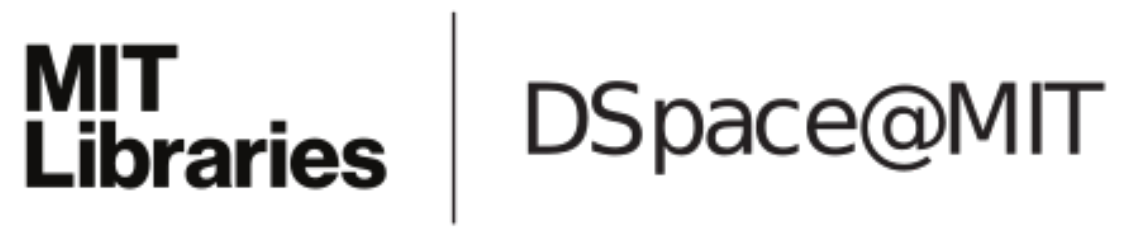

\author{
MIT Open Access Articles
}

\begin{abstract}
Nucleon electromagnetic form factors from lattice $Q C D$ using 2+1 flavor domain wall fermions on fine lattices and chiral perturbation theory
\end{abstract}

The MIT Faculty has made this article openly available. Please share how this access benefits you. Your story matters.

Citation: Syritsyn, S. N., et al. (2010). Nucleon electromagnetic form factors from lattice QCD using $\$ 2+1 \$$ flavor domain wall fermions on fine lattices and chiral perturbation theory. Phys. Rev. D 81: 034507/1-37. (c) 2010 The American Physical Society

As Published: http://dx.doi.org/10.1103/PhysRevD.81.034507

Publisher: American Physical Society

Persistent URL: http://hdl.handle.net/1721.1/58845

Version: Final published version: final published article, as it appeared in a journal, conference proceedings, or other formally published context

Terms of Use: Article is made available in accordance with the publisher's policy and may be subject to US copyright law. Please refer to the publisher's site for terms of use. 


\title{
Nucleon electromagnetic form factors from lattice QCD using $2+1$ flavor domain wall fermions on fine lattices and chiral perturbation theory
}

\author{
S. N. Syritsyn, ${ }^{1}$ J. D. Bratt, ${ }^{1}$ M. F. Lin, ${ }^{1}$ H. B. Meyer, ${ }^{1}$ J. W. Negele, ${ }^{1}$ A. V. Pochinsky, ${ }^{1}$ M. Procura, ${ }^{1}$ M. Engelhardt, ${ }^{2}$ \\ Ph. Hägler, ${ }^{3}$ T. R. Hemmert, ${ }^{4}$ and W. Schroers ${ }^{5}$
}

(LHPC Collaboration)

\author{
${ }^{1}$ Center for Theoretical Physics, Massachusetts Institute of Technology, Cambridge, Massachusetts 02139, USA \\ ${ }^{2}$ Physics Department, New Mexico State University, Las Cruces, New Mexico 88003-8001, USA \\ ${ }^{3}$ Institut für Theoretische Physik T39, Physik-Department der TU München, James-Franck-Straße, D-85747 Garching, Germany \\ ${ }^{4}$ Theoretische Physik, Universität Regensburg, D-93040 Regensburg, Germany \\ ${ }^{5}$ Institute of Physics, Academia Sinica, Taipei 115, Taiwan, R.O.C. \\ (Received 31 August 2009; published 11 February 2010)
}

\begin{abstract}
We present a high-statistics calculation of nucleon electromagnetic form factors in $N_{f}=2+1$ lattice QCD using domain wall quarks on fine lattices, to attain a new level of precision in systematic and statistical errors. Our calculations use $32^{3} \times 64$ lattices with lattice spacing $a=0.084 \mathrm{fm}$ for pion masses of 297,355 , and $403 \mathrm{MeV}$, and we perform an overdetermined analysis using on the order of 3600 to 7000 measurements to calculate nucleon electric and magnetic form factors up to $Q^{2} \approx 1.05 \mathrm{GeV}^{2}$. Results are shown to be consistent with those obtained using valence domain wall quarks with improved staggered sea quarks, and using coarse domain wall lattices. We determine the isovector Dirac radius $r_{1}^{v}$, Pauli radius $r_{2}^{v}$ and anomalous magnetic moment $\kappa_{v}$. We also determine connected contributions to the corresponding isoscalar observables. We extrapolate these observables to the physical pion mass using two different formulations of two-flavor chiral effective field theory at one loop: the heavy baryon small scale expansion and covariant baryon chiral perturbation theory. The isovector results and the connected contributions to the isoscalar results are compared with experiment, and the need for calculations at smaller pion masses is discussed.
\end{abstract}

DOI: 10.1103/PhysRevD.81.034507

PACS numbers: 12.38.Gc, 13.40.Gp, 14.20.Dh

\section{INTRODUCTION}

Electromagnetic form factors characterize fundamental aspects of the structure of protons and neutrons. In particular, they specify the spatial distribution of charge and magnetization. For nonrelativistic systems the electric and magnetic form factors would just be Fourier transforms of the charge and current densities. At each $Q^{2}$, the Sachs form factors $G_{E}\left(Q^{2}\right)$ and $G_{M}\left(Q^{2}\right)$ may be regarded as three-dimensional Fourier transforms of charge and magnetization densities defined in the corresponding Breit frame. A probabilistic interpretation of the Dirac and Pauli form factors $F_{1}\left(Q^{2}\right)$ and $F_{2}\left(Q^{2}\right)$ can be obtained from a two-dimensional Fourier transformation to impact parameter space in the infinite momentum frame [1,2]. At high momentum transfer, the elastic form factor specifies the amplitude for a single quark in the nucleon to absorb a very large momentum kick and share it with the other constituents in such a way that the nucleon remains in its ground state instead of being excited. It thus describes the onset of scaling and the scale at which quark counting rules become applicable, which is an unresolved theoretical question in nonperturbative QCD. The combination of precision experimental measurements and crisp theoretical interpretation renders elastic nucleon form factors particu- larly significant. Given the constantly improving experimental measurements of form factors and their fundamental significance, it is an important challenge for lattice QCD to calculate them accurately from first principles.

The nucleon Dirac and Pauli form factors, $F_{1}\left(Q^{2}\right)$ and $F_{2}\left(Q^{2}\right)$, respectively, are defined as follows for each quark flavor $(f)$ :

$$
\begin{aligned}
\left\langle P^{\prime}, S^{\prime}\left|V_{(f)}^{\mu}\right| P, S\right\rangle= & \bar{U}\left(P^{\prime}, S^{\prime}\right)\left[\gamma^{\mu} F_{1}^{(f)}\left(Q^{2}\right)\right. \\
& \left.+i \sigma^{\mu \nu} \frac{q_{\nu}}{2 M_{N}} F_{2}^{(f)}\left(Q^{2}\right)\right] U(P, S), \\
V_{(f)}^{\mu}= & \bar{\psi}_{(f)} \gamma^{\mu} \psi_{(f)},
\end{aligned}
$$

where $P, P^{\prime}$ are the initial and final nucleon momenta; $S, S^{\prime}$ are the corresponding spin vectors; the momentum transfer is $q=P^{\prime}-P$ with $Q^{2}=-q^{2} \geq 0$; and $M_{N}$ is the nucleon mass. The Sachs form factors $G_{E}\left(Q^{2}\right)$ and $G_{M}\left(Q^{2}\right)$ are defined by

$$
\begin{gathered}
G_{E}\left(Q^{2}\right)=F_{1}\left(Q^{2}\right)-\frac{Q^{2}}{\left(2 M_{N}\right)^{2}} F_{2}\left(Q^{2}\right), \\
G_{M}\left(Q^{2}\right)=F_{1}\left(Q^{2}\right)+F_{2}\left(Q^{2}\right) .
\end{gathered}
$$


Finally, it is useful to define isoscalar and isovector form factors as the sum and difference of proton and neutron form factors as follows:

$$
\begin{aligned}
F_{1,2}^{v}\left(Q^{2}\right)= & F_{1,2}^{p}\left(Q^{2}\right)-F_{1,2}^{n}\left(Q^{2}\right)=F_{1,2}^{u}\left(Q^{2}\right)-F_{1,2}^{d}\left(Q^{2}\right) \\
\equiv & F_{1,2}^{u-d}\left(Q^{2}\right), \\
F_{1,2}^{s}\left(Q^{2}\right) & =F_{1,2}^{p}\left(Q^{2}\right)+F_{1,2}^{n}\left(Q^{2}\right) \\
& =\frac{1}{3}\left(F_{1,2}^{u}\left(Q^{2}\right)+F_{1,2}^{d}\left(Q^{2}\right)\right) \equiv \frac{1}{3} F_{1,2}^{u+d}\left(Q^{2}\right),
\end{aligned}
$$

where $F_{i}^{p, n}$ are the form factors of the electromagnetic current in a proton and a neutron, respectively:

$$
\begin{aligned}
V_{\mathrm{em}, p}^{\mu} & =\frac{2}{3} \bar{u} \gamma^{\mu} u-\frac{1}{3} \bar{d} \gamma^{\mu} d, \\
V_{\mathrm{em}, n}^{\mu} & =-\frac{1}{3} \bar{u} \gamma^{\mu} u+\frac{2}{3} \bar{d} \gamma^{\mu} d .
\end{aligned}
$$

Although proton and neutron form factors contain both connected diagrams, calculated in this work, and disconnected diagrams, which are currently omitted, the disconnected diagrams do not contribute to the isovector form factors $F_{i}^{v}$. Hence, we will devote particular attention in this work to the isovector form factors.

Precise experimental measurements of the set of all four nucleon form factors remains challenging, and the field is marked both by significant recent developments and open questions. Although the most straightforward measurement is $F_{1}\left(Q^{2}\right)$ for the proton, the slope at very small values of $Q^{2}$ remains controversial. Phenomenological fits to experimental form factors $[3,4]$ appear to be inconsistent with analyses based on dispersion theory [5-7], with phenomenological fits yielding larger Dirac radii. Hence, a new generation of precision measurements of form factors at low momentum transfer is currently being undertaken at Mainz [8]. Spin polarization experiments [913] yielded results for $F_{2}\left(Q^{2}\right)$ significantly different from traditional measurements based on Rosenbluth separation, and there is a consensus that two-photon exchange processes contribute much more strongly to the backward cross section used in Rosenbluth separation than to polarization transfer [4]. However, there are not yet precise theoretical calculations of two-photon exchange that fully resolve the discrepancy between the two experimental methods, and hence experiments using positron scattering, for which the relative contribution of the two-photon term changes sign, are being prepared $[14,15]$. Neutron form factors are more uncertain than proton form factors because of the need to know the nuclear wave function to go from experimental scattering results from deuterium or ${ }^{3} \mathrm{He}$ to a statement about the neutron form factor. Over the years, nuclear models and theoretical calculations have been refined, but it is still a challenge to provide a definitive estimate of the uncertainty in the claimed neutron form factors extracted from nuclear targets. Given the level of precision to which we aspire in lattice calculations, systematic uncertainties in isovector and isoscalar form factors are not necessarily negligible. In the future when lattice calculations reliably include precise calculations of disconnected contributions, it may well be that lattice calculations play a role in guiding the resolution of some of these experimental questions.

Electromagnetic form factors have now been calculated in lattice QCD using a variety of actions. Quenched calculations of form factors have used both Wilson $[16,17]$ and domain wall [18] fermion actions, and additional quenched calculations have addressed magnetic moments and rootmean-squared (rms) radii $[19,20]$. Dynamical calculations with two flavors have used Wilson [17], clover improved Wilson [21], twisted-mass [22,23] and domain wall [24] actions. Extensive $2+1$ flavor calculations have been performed with a mixed action, which combines domain wall valence quarks and improved staggered sea quarks [25-27], using the same methodology as in the present work, and comparisons will be made to assess the consistency of the full domain wall and mixed-action results. Dynamical domain wall results with $2+1$ flavors on coarse lattices with $a=0.114 \mathrm{fm}$ have recently been reported [26,28,29], and initial results from the present work on fine lattices with $a=0.084 \mathrm{fm}$ were presented in Ref. [30].

The goal of this work is to achieve a new level of precision in calculating form factors from first principles in lattice QCD. Hence, we have done everything feasible within the constraints of our computational resources to reduce both statistical and systematic errors. Since this involves a number of new developments, we describe our methodology, innovations, and tests in detail. Because the signal to noise for baryon observables degrades with increasing Euclidean time $t$ as $e^{-\left(M_{n}-3 / 2 m_{\pi}\right) t}$, we have obtained high statistics using from 3688 to 7064 measurements of operators at a given mass by performing 8 measurements per lattice and have verified their statistical independence. The source-sink separation distance is a crucial issue, since an excessively large distance degrades the statistical accuracy whereas too small a distance introduces systematic errors from the contributions of excited states. We present a quantitative analysis of the contributions of excited states, and using this analysis, provide compelling numerical evidence that with our choice, which has been questioned in the literature [29], excited state contributions are negligible in our present work. Our overdetermined analysis of form factors provides a general framework for optimizing the precision of our lattice calculations by combining measurements of as many distinct nucleon matrix elements involving the form factors at the same $Q^{2}$ value as practical. We also describe how we choose which contributions to include, and treat error correlations. We compare domain wall calculations on fine lattices at three masses with a calculation on a 
coarse lattice at one mass, and present evidence that the $\mathcal{O}\left(a^{2}\right)$ corrections are indeed small. We also compare our results with mixed-action results, showing essential consistency between mixed and domain wall actions and emphasizing the small size of finite-volume corrections to calculations on a $2.5 \mathrm{fm}$ lattice at $m_{\pi}=350 \mathrm{MeV}$ that have been calculated to high precision with the mixed action. We perform chiral extrapolations of the Dirac and Pauli isovector $(v)$ and isoscalar $(s)$ mean squared radii, $\left(r_{1}^{v, s}\right)^{2}$ and $\left(r_{2}^{v, s}\right)^{2}$, respectively, and of the anomalous magnetic moments $\kappa_{v, s}$. We use two different formulations of $S U$ (2) chiral effective field theory: the heavy baryon small scale expansion (SSE) which includes explicit $\Delta(1232)$ degrees of freedom [31] and covariant baryon chiral perturbation theory (BChPT) without an explicit $\Delta(1232)$ in the IR-scheme $[32,33]$, which represents a variant of infrared regularization [34]. ${ }^{1}$ We explore the degree to which the relevant low-energy constants can be determined in the range of masses we consider and the variation of the extrapolated results in both schemes. We conclude that with the new level of precision we achieve, it is necessary to extend the lattice calculations to substantially lower masses to make contact with the regime of applicability of chiral effective theory and possibly reach agreement with experiment.

The remainder of this paper is organized as follows. In Sec. II, we present a detailed description of our methodology, including setting the scale, computation of nucleon matrix elements and coherent sink technique, optimization of sources, treatment of error correlations and constraints in the overdetermined analysis, and a check of the independence of multiple measurements per configuration. Section III presents the results of our lattice calculations for isovector form factors, including phenomenological fits to the momentum transfer dependence and determination of the Dirac radius $\left(r_{1}^{v}\right)^{2}$, the Pauli radius $\left(r_{2}^{v}\right)^{2}$, and the anomalous magnetic moment $\kappa_{v}$. Comparisons are made with domain wall calculations on a coarse lattice and with mixed-action calculations using valence domain wall valence quarks and improved staggered sea quarks. We also present the chirally extrapolated values of $\left(r_{1}^{v}\right)^{2},\left(r_{2}^{v}\right)^{2}$, and $\kappa_{v}$ to the physical pion mass using the SSE and covariant chiral effective field theories and compare them with experiment. Corresponding results for isoscalar form factors are presented in Sec. IV. Systematic errors are discussed in Sec. V, results are compared with other work in Sec. VI, and conclusions and opportunities for further understanding of nucleon form factors are discussed in Sec. VII. Selected numerical results are tabulated in Appendix A and the optimized sources are described in Appendix B.

\footnotetext{
${ }^{1}$ For recent work on chiral extrapolations of nucleon magnetic form factors and octet-baryon charge radii in heavy baryon ChPT with finite range regularization, we refer the reader to Refs. [35,36].
}

\section{LATTICE METHODOLOGY}

\section{A. Dynamical domain wall ensembles}

In our calculations, we analyze gauge configurations generated by the RBC and UKQCD Collaborations [37] with the Iwasaki gauge action and $N_{f}=2+1$ flavors of dynamical domain wall fermions. The gauge configuration ensembles are summarized in Table I. We obtain the relevant physical results from three fine lattice ensembles with lattice spacing $a=0.084 \mathrm{fm}$. We use one coarse lattice ensemble with known lattice spacing $a=0.114 \mathrm{fm}$ [37] to set the scale on the fine lattices and control the systematic errors due to discretization.

In our analysis, we use only a unitary fermion action, where the sea and valence fermion actions and masses are exactly the same. The extent of the fifth dimension is chosen to be $L_{s}=16$, which keeps the residual mass $m_{\text {res }}$ smaller than the bare quark masses for all ensembles.

In order to maximize the signal to noise ratio and suppress excited state contamination, we carefully optimize the quark propagator sources. We use Wuppertal smearing $[38,39]$ of quark sources combined with APE smearing [40] of the source gauge fields to reach the maximum overlap of the lattice nucleon operators with the nucleon ground state and reduce its fluctuation. The details of optimization and the source parameters we use are given in Appendix B.

To increase statistics, we perform eight measurements of nucleon correlation functions on each gauge configuration. To do so, we compute four forward quark propagators and construct nucleon and antinucleon correlators advancing in the positive and negative time directions, respectively. The data for antinucleons are transformed according to the reflection symmetry and combined with the data for nucleons into a single data set. We save computing time by using the "coherent" backward propagator technique, in which we compute only a sum of four backward propagators for four separate sequential sources with the same hadron type, flavor, and sink momentum. To check for possible systematic effects, we recalculate the nucleon three-point functions using independent backward propagators and larger source-sink separation on a subset of our lightest pion ensemble, and the extracted form factors (see Fig. 18) show no significant deviation from the method we use. Since lattice data may be autocorrelated, we block all the measurements on the two consecutive gauge configurations, and also check that the measurements we get are indeed independent by increasing the block size to include eight consecutive configurations (see Fig. 2).

\section{B. Pion mass, decay constant, and setting the fine lattice scale}

So far, the scale has been set only for the coarse lattice ensembles [37]. In order to set the scale for the fine lattice ensembles, we compare the lattice values for the pion 
TABLE I. Gauge configuration ensembles used for our analysis, with one coarse and three fine lattice spacings. These configurations were generated by the RBC and UKQCD [37] Collaborations. The coarse lattice spacing was determined in [37], and the fine lattice spacing is determined in Sec. II B. Measurement count (\# meas.) includes a factor of 8 for each gauge configuration. Note that for $m_{\pi}$, $F_{\pi}, m_{\text {res }}^{\prime}$ the measurement count is the number of configurations multiplied by 4 instead of $8 . T$ is the source-sink separation in lattice units.

\begin{tabular}{lcrccccccccc}
\hline \hline$L_{s}^{3} \times L_{t}$ & $a[\mathrm{fm}]$ & $T$ & \# meas. & $a m_{l} / a m_{h}$ & $a m_{\mathrm{res}}^{\prime} \times 10^{3}$ & $a m_{\pi}$ & $m_{\pi}[\mathrm{MeV}]$ & $a F_{\pi}$ & $F_{\pi}[\mathrm{MeV}]$ & $a M_{N}$ & $M_{N}[\mathrm{MeV}]$ \\
\hline $24^{3} \times 64$ & 0.114 & 9 & 3208 & $0.005 / 0.04$ & $3.15(1)$ & $0.1901(3)$ & $329(5)$ & $0.06100(11)$ & $105.5(1.7)$ & $0.657(4)$ & $1136(20)$ \\
$32^{3} \times 64$ & 0.084 & 12 & 4928 & $0.004 / 0.03$ & $0.665(3)$ & $0.1268(3)$ & $297(5)$ & $0.04400(15)$ & $102.9(1.8)$ & $0.474(4)$ & $1109(21)$ \\
$32^{3} \times 64$ & 0.084 & 12 & 7064 & $0.006 / 0.03$ & $0.663(2)$ & $0.1519(3)$ & $355(6)$ & $0.04571(09)$ & $107.0(1.8)$ & $0.501(2)$ & $1172(21)$ \\
$32^{3} \times 64$ & 0.084 & 12 & 4224 & $0.008 / 0.03$ & $0.668(3)$ & $0.1724(3)$ & $403(7)$ & $0.04755(18)$ & $111.3(2.0)$ & $0.522(2)$ & $1221(21)$ \\
\hline \hline
\end{tabular}

decay constant $\left(a F_{\pi}\right)$ on coarse and fine lattices at the same value of the dimensionless ratio $\left(m_{\pi} / F_{\pi}\right)^{2}$ ignoring possible finite lattice spacing effects in the pion decay constant $F_{\pi}$.

First, we compute the pion mass, the pion decay constant, and the local axial current renormalization constant ${ }^{2}$ from fits to the pseudoscalar density and axial current correlators using the PCAC relation [42]:

$$
\begin{aligned}
\left\langle A_{0}(t, \vec{p}=0) \tilde{J}_{5}(0)\right\rangle= & \left(e^{-m_{\pi} t}-e^{-m_{\pi}\left(L_{t}-t\right)}\right) \times \frac{F_{\pi}^{2} m_{\pi}^{2}}{2\left(m_{l}+m_{\mathrm{res}}^{\prime}\right)} \\
& \times Z_{A}^{-1} Z_{\mathrm{sm}}^{-1}, \\
\left\langle J_{5 q}(t, \vec{p}=0) \tilde{J}_{5}(0)\right\rangle= & \left(e^{-m_{\pi} t}+e^{-m_{\pi}\left(L_{t}-t\right)}\right) \\
& \times \frac{F_{\pi}^{2} m_{\pi}^{3}}{4\left(m_{l}+m_{\mathrm{res}}^{\prime}\right)^{2}} \times m_{\mathrm{res}}^{\prime} Z_{\mathrm{sm}}^{-1}
\end{aligned}
$$

where $A_{0}$ is the local axial charge, $J_{5 q}$ is the fifth dimension midpoint pseudoscalar density, $J_{5}\left(\tilde{J}_{5}\right)$ is the (smeared) pseudoscalar density, and $m_{\text {res }}^{\prime}$ is the domain wall residual mass at a given quark mass as measured by the ratio $\left\langle J_{5 q}(t) \tilde{J}_{5}(0)\right\rangle /\left\langle\tilde{J}_{5}(t) \tilde{J}_{5}(0)\right\rangle$ in Ref [42]. The pion decay constant $F_{\pi}$ convention is such that

$$
F_{\pi}^{\text {phys }}=92.4 \pm 0.3 \mathrm{MeV} \text {. }
$$

We choose the range of $t$ to be [12:52] to exclude any excited state contaminations. We define the smearing

\footnotetext{
${ }^{2}$ In this paper, we assume that the renormalization constant $Z_{\mathcal{A}}$ of the (partially) conserved domain wall axial current $\mathcal{A}_{\mu}$ is equal to its $L_{s} \rightarrow \infty$ value of one, and note that the finite $L_{s}$ deviation has been estimated in $[37,41]$ to give $\left|Z_{\mathcal{A}}-1\right| \lesssim 1 \%$. The values of $F_{\pi}$ that we compute are, in fact, $F_{\pi} / Z_{\mathcal{A}}$.
}

renormalization constant $Z_{\mathrm{sm}}$ from the plateau $\left\langle J_{5}(t) \tilde{J}_{5}(0)\right\rangle /\left\langle\tilde{J}_{5}(t) \tilde{J}_{5}(0)\right\rangle$ and the local axial current renormalization constant $Z_{A}$ from the ratio of $\left\langle\mathcal{A}_{0}(t+\right.$ $\left.1 / 2) \tilde{J}_{5}(0)\right\rangle$ and $\left\langle A_{0}(t) \tilde{J}_{5}(0)\right\rangle$ appropriately averaged to suppress $O(a)$ effects due to $a / 2$ displacement of the conserved axial current $\mathcal{A}_{0}(t+1 / 2)$ [42]. The results for $a m_{\pi}, a F_{\pi}$, and $a m_{\text {res }}^{\prime}$ are shown in Table I. The error bars reflect both the statistical error and the systematic error due to different fitting ranges.

Second, we fit $m_{\pi}$ and $F_{\pi}$ at three values of the light quark mass using $\mathcal{O}\left(p^{4}\right) S U(2)$ chiral perturbation theory $[43,44]$

$$
\begin{aligned}
& a^{2} m_{\pi}^{2}=a^{2} \chi\left\{1+\frac{2 a^{2} \chi}{(a F)^{2}} l_{3}^{r}\left(a^{-1}\right)+\frac{a^{2} \chi}{32 \pi^{2}(a F)^{2}} \log \left(a^{2} \chi\right)\right\}, \\
& a F_{\pi}=a F\left\{1+\frac{a^{2} \chi}{(a F)^{2}} l_{4}^{r}\left(a^{-1}\right)-\frac{a^{2} \chi}{16 \pi^{2}(a F)^{2}} \log \left(a^{2} \chi\right)\right\},
\end{aligned}
$$

where $a^{2} \chi=2(a B) \cdot a\left(m_{l}+m_{\mathrm{res}}^{\prime}\right), l_{3,4}^{r}\left(a^{-1}\right)$ are the nextto-leading-order (NLO) low-energy constants (LECs) at the scale $\Lambda=a^{-1}$, and the fit variables are $(a F),(a B)$, and $l_{3,4}^{r}$. However, the fit is not satisfactory in terms of $\chi^{2}$ : for 2 degrees of freedom, we get $\chi^{2} \approx 7$, with its probability to be this or higher being $\lesssim 3 \%$. This is the first of many indications that chiral perturbation theory, at the order we can use, is not accurate in the range of masses we are considering. Hence, the LEC's are not precisely determined although, as noted below, we obtain an adequate interpolation to set the scale.

The NLO LECs $l_{3,4}^{r}$ from our fit can be converted to the scale-independent parameters $\bar{l}_{3,4}$ [43]. At the physical pion mass we obtain

$$
\bar{l}_{3}=3.08(11), \quad \bar{l}_{4}=4.24(4) .
$$

Our results for $\bar{l}_{3}$ and $\bar{l}_{4}$ are in excellent agreement with the calculations done with the coarse lattice spacing [37] by the RBC and UKQCD Collaborations who obtained $\bar{l}_{3}=$ $3.13(33)$ and $\bar{l}_{4}=4.43(14)$, implying that the discretization errors may be small. Our result for $\bar{l}_{3}$ is also in agreement with the crude estimate $\bar{l}_{3}=2.9 \pm 2.4$ [43], 
and with the lattice determination $\bar{l}_{3}=3.0(5)(1)$ [45] using $N_{f}=2$ dynamical Wilson fermions. However, it disagrees with $\bar{l}_{3}=3.42(8)(10)$ (the errors are statistical and systematic due to residual lattice artifacts) from the ETM Collaboration [46]. This discrepancy could arise from the difference between $N_{f}=2$ and $N_{f}=3$ flavors of dynamical fermions. Furthermore, our result for $\bar{l}_{4}$ agrees with the value $\bar{l}_{4}=4.3 \pm 0.9$ obtained by Gasser and Leutwyler [43]. This estimate has been sharpened in [47]: $\bar{l}_{4}=4.4 \pm$ 0.2 , which agrees with the value $\bar{l}_{4}=4.4 \pm 0.3$ obtained by Bijnens et al. [48]. The ETM Collaboration result [46] is $\bar{l}_{4}=4.59(4)(2)$, which again disagrees with ours, presumably for the same reason.

The resulting interpolated functional dependence of $\left(a F_{\pi}\right)$ on $\left(m_{\pi} / F_{\pi}\right)^{2}$ is shown in Fig. 1. To determine the lattice spacing on the fine ensembles, we compare the dimensionless quantity $\left(a F_{\pi}\right)$ on the coarse and fine ensembles at the same value of $\left(m_{\pi} / F_{\pi}\right)^{2}=\left.\left(m_{\pi} / F_{\pi}\right)^{2}\right|_{\text {coarse }}$. Since we do not have a fine ensemble with $\left(m_{\pi} / F_{\pi}\right)^{2}$ value corresponding exactly to the coarse ensemble, we take the interpolated value from the functional dependence on Fig. 1. To check for systematic effects, we also interpolate $\left(a F_{\pi}\right)$ linearly in $\left(m_{\pi} / F_{\pi}\right)^{2}$ between the two lightest pion masses, and this method gives a consistent result (see Fig. 1). We also compare the coarse and fine lattice results for the nucleon mass $\left(a M_{N}\right)$ in the same way:

$$
\frac{\left.\left(a F_{\pi}\right)\right|_{\text {fine }}}{\left.\left(a F_{\pi}\right)\right|_{\text {coarse }}}=0.735(2), \quad \frac{\left.\left(a M_{N}\right)\right|_{\text {fine }}}{\left.\left(a M_{N}\right)\right|_{\text {coarse }}}=0.742(5) .
$$

Although these ratios are barely consistent within errors, their discrepancy is irrelevant to the fine scale determination as long as the fractional error in the coarse lattice scale $a_{\text {coarse }}=0.1141(18) \mathrm{fm}$ dominates. We obtain the value for the fine lattice scale

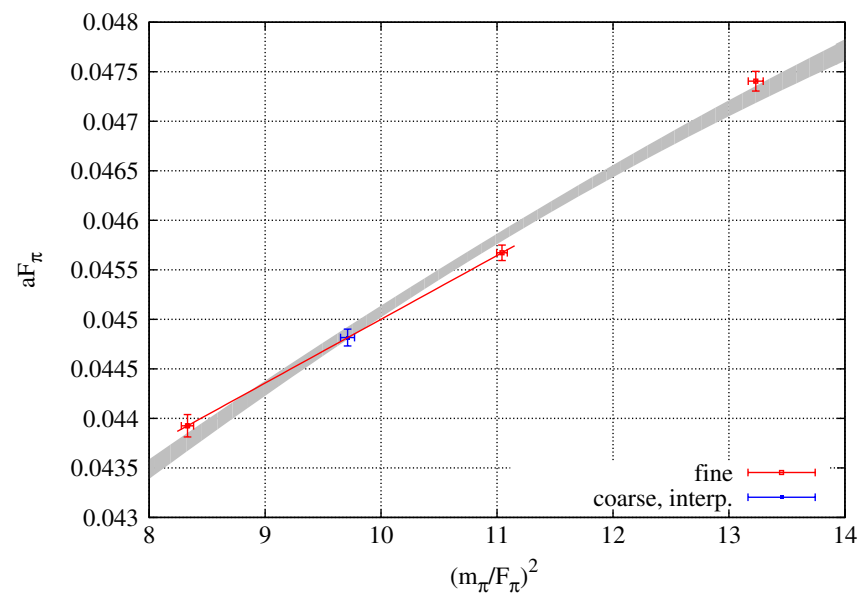

FIG. 1 (color online). One-loop $S U(2)$ ChPT interpolation of the fine lattice values of $F_{\pi}$ and $m_{\pi}$. The point with abscissa $9.71=\left(m_{\pi} / F_{\pi}\right)_{\text {coarse }}^{2}$ was obtained by interpolating $\left(a F_{\pi}\right)$ linearly in $\left(m_{\pi} / F_{\pi}\right)^{2}$.

$$
a_{\text {fine }}=0.0840(14) \mathrm{fm}, \quad a_{\text {fine }}^{-1}=2.34(4) \mathrm{GeV} \text {. }
$$

\section{Extraction of nucleon matrix elements}

In order to calculate nucleon matrix elements, we compute the three-point polarized nucleon correlators involving the vector current, along with the two-point correlators [25]:

$$
\begin{aligned}
& C_{2 \mathrm{pt}}(t, P)=\sum_{\vec{x}} e^{-i \vec{P} \cdot \vec{x}} \sum_{\alpha \beta}\left(\Gamma_{\mathrm{pol}}\right)_{\alpha \beta}\left\langle N_{\beta}(\vec{x}, t) \bar{N}_{\alpha}(0,0)\right\rangle, \\
& C_{3 \mathrm{pt}}^{V^{\mu}}\left(\tau, T ; P, P^{\prime}\right)=\sum_{\vec{x}, \vec{y}} e^{-i \vec{P}^{\prime} \cdot \vec{x}+i\left(\vec{P}^{\prime}-\vec{P}\right) \cdot \vec{y}} \sum_{\alpha \beta}\left(\Gamma_{\mathrm{pol}}\right)_{\alpha \beta} \\
& \times\left\langle N_{\beta}(\vec{x}, T) V^{\mu}(\vec{y}, \tau) \bar{N}_{\alpha}(0,0)\right\rangle,
\end{aligned}
$$

where $N_{\beta}, \quad \bar{N}_{\alpha}$ are the lattice nucleon operators; $\left\langle\Omega\left|N_{\alpha}(x)\right| P, \sigma\right\rangle=\sqrt{Z(P)} U_{\alpha}^{(\sigma)}(P) e^{-i P x}$, with $Z(P)$ parameterizing the overlap with the nucleon ground state; $\left(\Gamma_{\mathrm{pol}}\right)_{\alpha \beta}=\frac{1+\gamma_{4}}{2} \frac{1-i \gamma_{3} \gamma_{5}}{2}$ is the spin and parity projection matrix ${ }^{3}$; and $V^{a \mu}=\bar{q} \gamma^{\mu} t^{a} q$ is the vector current operator, where $t^{a}$ denotes an isospin generator. In the transfer matrix formalism, these correlators take the form

$$
\begin{aligned}
C_{2 \mathrm{pt}}(t, P)= & \frac{Z(P) e^{-E t}}{2 E} \operatorname{Tr}\left[\Gamma_{\mathrm{pol}}\left(i \not P+M_{N}\right)\right] \\
& + \text { excited states, }
\end{aligned}
$$

$$
\begin{aligned}
C_{3 \mathrm{pt}}^{V^{\mu}}\left(\tau, T ; P, P^{\prime}\right)= & \frac{\sqrt{Z\left(P^{\prime}\right) \cdot Z(P)} e^{-E^{\prime}(T-\tau)-E \tau}}{2 E^{\prime} \cdot 2 E} \\
& \times \operatorname{Tr}\left[\Gamma_{\mathrm{pol}}\left(i \not P^{\prime}+M_{N}\right)\right. \\
& \left.\times \Gamma^{\mu}\left(P^{\prime}, P\right)\left(i \not P+M_{N}\right)\right] \\
& + \text { excited states, }
\end{aligned}
$$

where $E$ and $E^{\prime}$ are the ground state energies of the initial and final nucleon states and $\Gamma^{\mu}\left(P^{\prime}, P\right)$ is the electromagnetic vertex function defined below in Eq. (24). Excited state contributions have generally similar forms with different $Z$-factors, vertices, and higher energies $E_{\text {exc }}>E$. The systematic effects related to them will be discussed in Sec. VA.

In order to extract the combinations of matrix elements $\left\langle P^{\prime}, S^{\prime}\left|V^{\mu}\right| P, S\right\rangle=\bar{U}\left(P^{\prime}, S^{\prime}\right) \Gamma^{\mu}\left(P^{\prime}, P\right) U(P, S)$, we combine the lattice nucleon correlators (19) and (20) into the usual ratio of three- and two-point correlation functions, which we find useful to write in a convenient and illuminating new form as follows. First, we define two ratios, a normalization ratio, $R_{N}$, and an asymmetry ratio, $R_{A}$,

\footnotetext{
${ }^{3}$ In this subsection, we use Euclidean $\gamma$-matrices, $\left(\gamma^{\mu}\right)^{\dagger}=$ $\gamma^{\mu},\left\{\gamma^{\mu}, \gamma^{\nu}\right\}=2 \delta^{\mu \nu}$.
} 


$$
\begin{gathered}
R_{N} \equiv \frac{C_{3 \mathrm{pt}}^{V^{\mu}}\left(\tau, T ; P, P^{\prime}\right)}{\sqrt{C_{2 \mathrm{pt}}(T, P) C_{2 \mathrm{pt}}\left(T, P^{\prime}\right)}}, \\
R_{A} \equiv \sqrt{\frac{C_{2 \mathrm{pt}}(T-\tau, P) C_{2 \mathrm{pt}}\left(\tau, P^{\prime}\right)}{C_{2 \mathrm{pt}}\left(T-\tau, P^{\prime}\right) C_{2 \mathrm{pt}}(\tau, P)}} .
\end{gathered}
$$

The physical matrix element is then given by the product

$$
\begin{aligned}
R^{V^{\mu}} & \equiv R_{N} R_{A} \\
& =\frac{C_{3 \mathrm{pt}}^{V^{\mu}}\left(\tau, T ; P, P^{\prime}\right)}{\sqrt{C_{2 \mathrm{pt}}(T, P) C_{2 \mathrm{pt}}\left(T, P^{\prime}\right)}} \sqrt{\frac{C_{2 \mathrm{pt}}(T-\tau, P) C_{2 \mathrm{pt}}\left(\tau, P^{\prime}\right)}{C_{2 \mathrm{pt}}\left(T-\tau, P^{\prime}\right) C_{2 \mathrm{pt}}(\tau, P)}} \stackrel{\{T, \tau, T\} \rightarrow \infty}{\longrightarrow} \frac{\sum_{S, S^{\prime}}\left(\bar{U}(P, S) \Gamma_{\mathrm{pol}} U\left(P^{\prime}, S^{\prime}\right)\right) \cdot\left\langle P^{\prime}, S^{\prime}\left|V_{\mu}\right| P, S\right\rangle}{\sqrt{2 E\left(E+M_{N}\right) \cdot 2 E^{\prime}\left(E^{\prime}+M_{N}\right)}} .
\end{aligned}
$$

The normalization ratio $R_{N}$ has the property that all the lattice-dependent overlap factors $Z$ for the ground state cancel out, which motivates its name, and yields the full result in the case of forward matrix elements $P=P^{\prime}$. The asymmetry ratio $R_{A}$ compensates the asymmetric exponential $\tau$ dependence of the three-point correlator, which motivates its name. In the absence of excited states, it would be equal to $\exp \left[-\left(E^{\prime}-E\right)(\tau-T / 2)\right]$ and in the forward case, $P^{\prime}=P$, this ratio is trivial and equal to one. In the general case, $P^{\prime} \neq P$, this ratio is still identically one in the center of the plateau, $\tau=T / 2$, and possesses the following symmetry around the plateau center: $R_{A}(T-$ $\tau)=1 / R_{A}(\tau)$.

The limits $\{T, \tau, T-\tau\} \rightarrow \infty$ should be taken to get rid of the excited state contamination. In practice, this requires adopting a value of source-sink separation $T$ large enough so that the excited state contributions to Eq. (23) are negligible compared to the other sources of errors and taking into account only points close to the center of plateaus. We will explicitly explore the contributions of excited states to $R^{V^{\mu}}$ in Sec. V, where the decomposition into the product $R_{N} R_{A}$ will prove extremely useful.

TABLE II. Momentum combinations used to extract the form factors (only one representative of in/out momenta is given). Approximate $Q^{2}$ values are given for the lightest $M_{N}=$ $1109 \mathrm{MeV}$.

\begin{tabular}{lcc}
\hline \hline$\#$ & $\langle$ out $|$ in $\rangle$ & $Q^{2}\left[\mathrm{GeV}^{2}\right]$ \\
\hline 1 & $\langle 0,0,0 \mid 0,0,0\rangle,\langle-1,0,0 \mid-1,0,0\rangle$ & 0.0 \\
2 & $\langle 0,0,0 \mid 1,0,0\rangle,\langle-1,0,0 \mid 0,0,0\rangle$ & 0.203 \\
3 & $\langle-1,0,0 \mid-1,0,1\rangle$ & 0.204 \\
4 & $\langle 0,0,0 \mid 1,1,0\rangle$ & 0.391 \\
5 & $\langle-1,0,0 \mid-1,1,1\rangle$ & 0.395 \\
6 & $\langle-1,0,0 \mid 0,0,1\rangle$ & 0.422 \\
7 & $\langle 0,0,0 \mid 1,1,1\rangle$ & 0.568 \\
8 & $\langle-1,0,0 \mid 0,1,1\rangle$ & 0.626 \\
9 & $\langle-1,0,0 \mid 1,0,0\rangle$ & 0.844 \\
10 & $\langle-1,0,0 \mid 1,1,0\rangle$ & 1.048 \\
\hline \hline
\end{tabular}

In order to obtain the most precise information on the form factors, we constrain the in- and out- lattice nucleon momenta to have components $0, \pm 1$. We list these momentum values in Table II. Higher momentum components are subject to stronger finite lattice spacing effects, i.e., discretization errors and dispersion relation deviations from the continuum expression. There is also an indication (see Sec. VA) that such states have larger excited state contaminations.

\section{Overdetermined analysis of form factors}

In Minkowski space, the nucleon electromagnetic vertex $\Gamma^{\mu}\left(P^{\prime}, P\right)$ in Eq. (20) is parameterized with two form factors:

$$
\begin{gathered}
\Gamma^{\mu}\left(P^{\prime}, P\right)=F_{1}\left(Q^{2}\right) \gamma^{\mu}+F_{2}\left(Q^{2}\right) \frac{i \sigma^{\mu \nu} q_{\nu}}{2 M_{N}}, \\
q=P^{\prime}-P, \quad Q^{2}=-q^{2} .
\end{gathered}
$$

Transforming the above expression to Euclidean space and substituting it into Eq. (20) and then Eq. (23) and neglecting the excited states, we obtain an overdetermined system of equations for the form factors $F_{1,2}\left(Q^{2}\right)$ at each fixed value of $Q^{2}$ :

$$
A_{\alpha i} F_{i}\left(Q^{2}\right)=R_{\alpha}^{V_{\mu}}, \quad \alpha=1,2, \ldots
$$

where we use a summation convention over $i=1,2$ and $\alpha$ is a composite index specifying the current component and the initial and final momenta of a given matrix element (for fixed $Q^{2}$ ), which will be discussed below. The right-hand side of Eq. (25) is evaluated using Eq. (23) with computed lattice correlators.

We find the solution of the overdetermined system from a linear fit, which minimizes the functional

$$
\mathcal{F}=\sum_{\alpha \beta}\left(A_{\alpha i} F_{i}-R_{\alpha}\right) C_{\alpha \beta}^{-1}\left(A_{\beta j} F_{j}-R_{\beta}\right),
$$

where $C_{\alpha \beta}$ is the covariance matrix of $R_{\alpha}$ averages, $C_{\alpha \beta}=$ $\frac{1}{N-1}\left(\left\langle\left\langle R_{\alpha} R_{\beta}\right\rangle\right\rangle-\left\langle\left\langle R_{\alpha}\right\rangle\right\rangle\left\langle\left\langle R_{\beta}\right\rangle\right\rangle\right)$, with the double brackets 


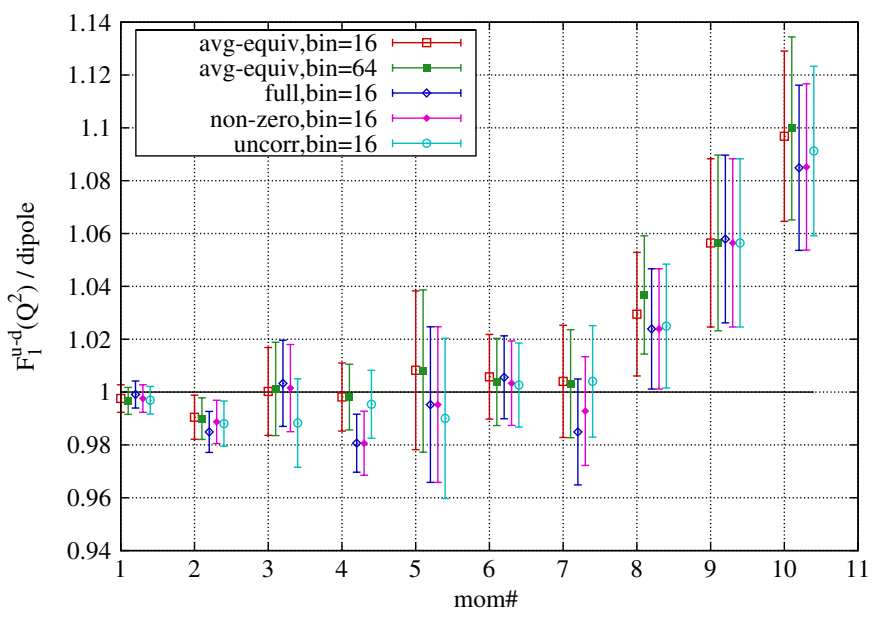

(a)

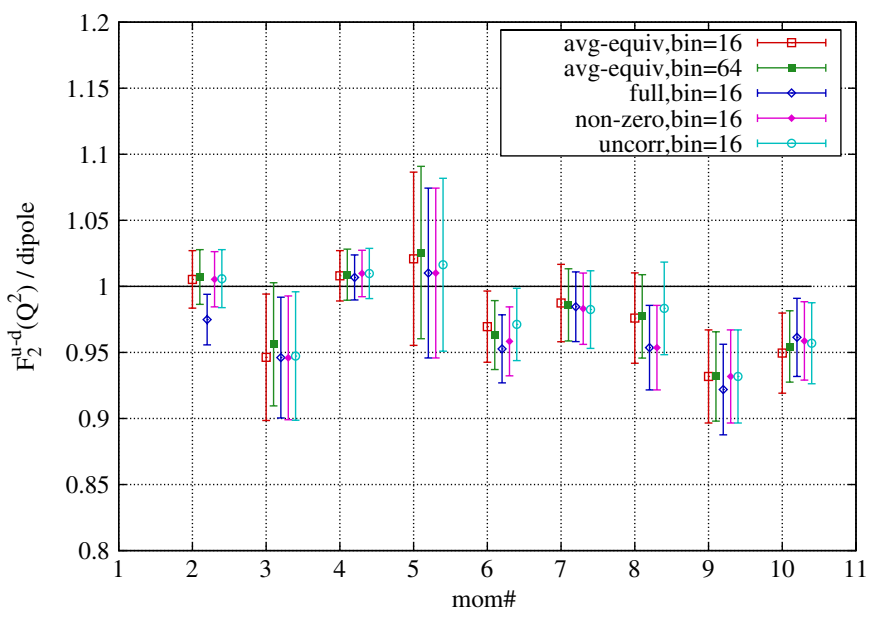

(b)

FIG. 2 (color online). Comparison of the nucleon Dirac (a) and Pauli (b) form factors extracted from the full overdetermined system (full), only nonzero equations (nonzero), uncorrelated fit (uncorr), and averaged equivalence classes (avg-equiv) for $m_{\pi}=297 \mathrm{MeV}$. Increased binning of data (eight successive configurations [bin $=64$ ] instead of two [bin $=16]$ ) shows no increase in estimation of statistical errors. Each form factor value is divided by the central value of the dipole fit. Table II lists the momentum combinations corresponding to each index on the horizontal axis.

denoting an ensemble average. Using the covariance matrix is crucial as long as the correlation functions prove to be correlated.

Since the covariance matrix may be ill determined, it can introduce uncontrollable errors into the extracted form factors. In general, a covariance matrix is notoriously difficult to reliably estimate in a statistical analysis. To make sure the linear fitting gives a correct result, we repeat the analysis with only the diagonal elements of the covariance matrix $C_{\alpha \alpha}$, which is equivalent to an uncorrelated linear fit. The comparison of these two schemes is presented in Fig. 2. We find that the form factors from an uncorrelated fit are consistent with the correlated fit results.

The overdetermined system (25) contains a subclass of equations which have an exactly zero left-hand side: $A_{\alpha i}=$ $0, i=1,2$. The measured lattice value of a right-hand side $R_{\alpha}$ is not required to be zero, and may be correlated with other matrix elements. In an uncorrelated fit, such equations decouple and do not contribute to the solution. In contrast, the outcome of a correlated fit depends on such values, thus potentially better utilizing the input from lattice calculations. In addition, by fitting the equations with a vanishing left-hand side, we check the symmetries of the electromagnetic vertex (24), statistically. Figure 2 also shows the agreement of the full overdetermined system solution and the system without zero left-hand side equations, confirming the consistency of our analysis.

The dimension of the overdetermined system may grow large, especially when many momentum combinations are included. For example, the most precise point for $Q^{2}>0$ corresponds to the matrix element $\left\langle 0,0,0\left|V^{\mu}(0)\right| 1,0,0\right\rangle$. All $V^{\mu}$ components, together with spatial rotations and reflections give 48 equations, only 16 of which are non- zero. It is useful to combine all the nucleon matrix elements for fixed $Q^{2}$ into equivalence classes based on spatial (rotational and reflection) symmetry. We adopted the following heuristic equivalence criteria ${ }^{4}$ for three-point functions:

(i) The momenta of the in- and out-states must be equivalent under the spatial symmetry.

(ii) The corresponding coefficients $A_{\alpha i}$ in Eq. (25) must be equal up to an overall sign.

(iii) The component of the current operator must be temporal or spatial and real or imaginary for both matrix elements being compared.

Blocking the measurements in each equivalence class is advantageous for two reasons. First, this reduces the dimension of the system of equations (25) and the covariance matrix we need to estimate, and we note that blocking strongly correlated values improves the covariance matrix condition number. Second, as long as for the equivalent three-point functions we need spatially equivalent twopoint functions to build the ratio in Eq. (23), we can block the two-point functions separately before computing the ratio. This improves the method in Eq. (23) by reducing the fluctuations of the two-point functions in the denominator.

To extract the final set of the form factors, we perform a correlated fit to the reduced (i.e., the system with no equations whose left-hand side is zero) overdetermined system with blocked equivalent equations.

\footnotetext{
${ }^{4}$ We have not classified the matrix elements according to the hypercubic lattice symmetry but instead use relations derived in the continuum. Thus these criteria may be thought of as numerical means to improve the condition number of the linear system we need to solve.
} 


\section{ISOVECTOR FORM FACTORS}

In experiments, the proton and neutron electromagnetic form factors [see Eq. (6)] are measured separately, and the isovector form factors (4) can be calculated by taking their difference. In lattice calculations, the Wick contractions of the quark fields in Eq. (6) with nucleon operators indicate that disconnected quark loops in the current insertion would be needed to calculate the proton and neutron form factors separately. The calculation of the disconnected quark loops is numerically demanding and has not been included in current calculations. However, the disconnected loop contributions cancel (in the isospin limit) in the contraction of the difference of the proton and neutron electromagnetic currents in Eq. (6), which gives the matrix elements needed for the isovector form factors. We focus our discussion on the isovector form factors in this section.

After presenting our lattice results for the isovector Dirac and Pauli form factors and the rms radii, we will compare chiral extrapolations using the SSE formulation and covariant baryon chiral perturbation theory. ${ }^{5}$ Corresponding results for the connected contributions to the isoscalar form factors will be presented in Sec. IV.

\section{A. Vector current renormalization}

The isovector Dirac form factor at zero momentum transfer, $F_{1}^{u-d}(0)$, gives the difference of the electric charges for the proton and neutron, which is 1 . Since we can measure $F_{1}^{u-d}(0)$ very accurately on the lattice, we use it to obtain the vector current renormalization constant, $Z_{V}$, by setting

$$
Z_{V} F_{1}^{u-d}(0)=1
$$

Since domain wall fermions have good chiral symmetry, in the chiral limit the vector current renormalization, $Z_{V}$, and the axial vector current renormalization, $Z_{A}$, are expected to be the same up to $O\left(a^{2}\right)$ corrections. $Z_{A}$ is measured by taking the ratio of the point-split five-dimensional conserved axial current to the local four-dimensional current (see Sec. II B and [42]). We show the results of $Z_{V}$ and $Z_{A}$ in Table III. Naive linear extrapolations in $m_{\pi}^{2}$ to the chiral limit show that $Z_{V}$ and $Z_{A}$ are consistent within errors, as is clearly shown in Fig. 3.

In the following analysis, we renormalize the form factors by $Z_{V}$ as measured on the corresponding ensemble. That is, we use a mass-dependent renormalization condition. The mass dependence of the renormalization constants is very mild and consistent with the theoretically expected form $Z_{V}\left(g_{0}\right)\left(1+b_{v} a m_{q}\right)$ [50]. The renormalized results for $F_{1}$ and $F_{2}$ with all the different flavor combi-

\footnotetext{
${ }^{5}$ For an analysis of nucleon electromagnetic form factors in $\mathrm{BChPT}$ with standard infrared regularization, we refer to [49].
}

TABLE III. Vector and axialvector current renormalization constants. The chiral limit values are obtained by linear extrapolations to $m_{\pi}^{2}=0$.

\begin{tabular}{lcc}
\hline \hline$m_{\pi}[\mathrm{MeV}]$ & $Z_{V}$ & $Z_{A}$ \\
\hline 297 & $0.7468(39)$ & $0.745025(24)$ \\
355 & $0.7479(22)$ & $0.745207(18)$ \\
403 & $0.7513(17)$ & $0.745317(20)$ \\
chiral limit & $0.7397(74)$ & $0.744700(55)$ \\
\hline \hline
\end{tabular}

nations are tabulated in Tables XIII, XIV, and $\mathrm{XV}$ in Appendix A.

\section{B. $Q^{2}$ dependence}

As will be discussed in the following section, ChPT describes the $Q^{2}$ dependence of the form factors for values of $Q^{2}$ much less than the chiral symmetry breaking scale (typically of the order of the nucleon mass). Lacking a model-independent functional form applicable in the large- $Q^{2}$ region, we study the $Q^{2}$ dependence using the phenomenological dipole or tripole formula. The Dirac form factor is fixed to 1 at $Q^{2}=0$ under our renormalization scheme, and we use the following one-parameter dipole or tripole formula to describe the $Q^{2}$ dependence:

$$
\begin{aligned}
& F_{1}\left(Q^{2}\right)=\frac{1}{\left(1+\frac{Q^{2}}{M_{D}^{2}}\right)^{2}} \quad \text { (one-parameter dipole), } \\
& F_{1}\left(Q^{2}\right)=\frac{1}{\left(1+\frac{Q^{2}}{M_{T}^{2}}\right)^{3}} \quad \text { (one-parameter tripole). }
\end{aligned}
$$

The Pauli form factor at $Q^{2}=0, F_{2}(0)$, cannot be mea-

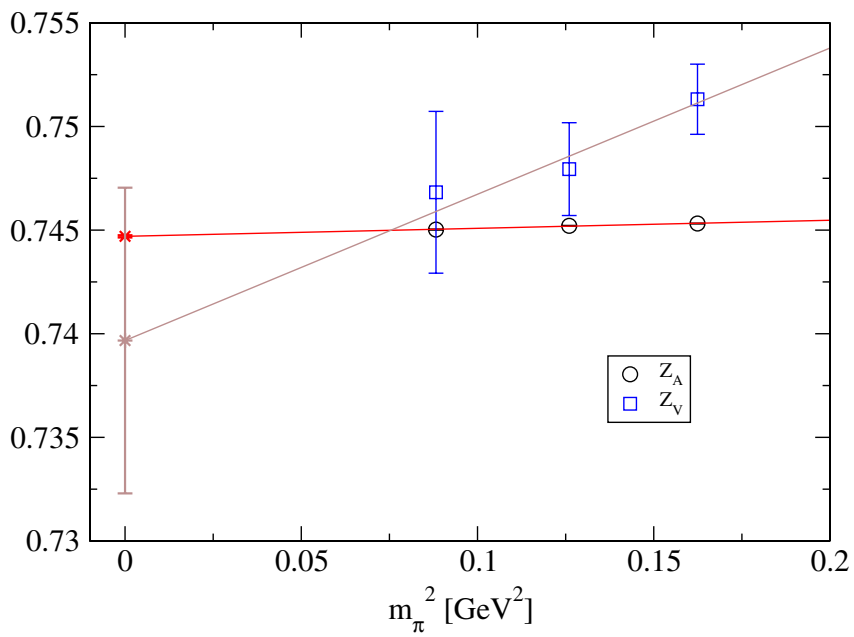

FIG. 3 (color online). Comparison of the vector and axial vector current renormalization constants. In the chiral limit, these two renormalization constants agree within errors. The errors on all the $Z_{A}$ points given in Table III are too small to appear on the figure. 
sured on the lattice directly. We thus fit the data using the two-parameter dipole or tripole formula,

$$
\begin{aligned}
& F_{2}\left(Q^{2}\right)=\frac{F_{2}(0)}{\left(1+\frac{Q^{2}}{M_{D}{ }^{2}}\right)^{2}} \quad \text { (two-parameter dipole) } \\
& F_{2}\left(Q^{2}\right)=\frac{F_{2}(0)}{\left(1+\frac{Q^{2}}{M_{T}^{2}}\right)^{3}} \quad \text { (two-parameter tripole) }
\end{aligned}
$$

We are interested in mean squared Dirac and Pauli radii, which are defined by the slope of the form factors at small $Q^{2}$ :

$$
F_{1,2}\left(Q^{2}\right)=F_{1,2}(0)\left[1-\frac{1}{6}\left(r_{1,2}\right)^{2} Q^{2}+\mathcal{O}\left(Q^{4}\right)\right],
$$

and are related to the pole masses by

$$
(r)^{2}=\frac{12}{M_{D}^{2}},
$$

for the dipole fits, and

$$
(r)^{2}=\frac{18}{\left(M_{T}\right)^{2}},
$$

for the tripole fits.

Note that results at different $Q^{2}$ from the same ensemble may be highly correlated [26], therefore we perform correlated least- $\chi^{2}$ fits to the data. We investigate the extent to which the dipole and tripole Ansätze describe our data and the stability of the fits by varying the maximum $Q^{2}$ values included in the fits.

In Table XVI we show the fit results for $F_{1}^{u-d}\left(Q^{2}\right)$ using the one-parameter dipole and tripole formulas in Eqs. (28) and (29). Comparing the $\chi^{2} /$ dof for the dipole and tripole fits, we see that the dipole fits are slightly preferred when larger $Q^{2}$ values are included in the fits. However, the Dirac radii determined from both the dipole and tripole fits agree within errors. In general, the dipole form describes the data reasonably well throughout the whole $Q^{2}$ range for all but one ensemble, the $m_{\pi}=355 \mathrm{MeV}$ ensemble, where, when $Q^{2}$ cutoff is larger than $0.3 \mathrm{GeV}^{2}$, $\chi^{2} /$ dof becomes very large. This may be due to the fact that this ensemble has the most statistics, and we start to see the deviation from the phenomenological dipole formula. For the other two ensembles, we can see the general trend that when large $Q^{2}$ points are included in the fits, the $\chi^{2} /$ dof becomes slightly worse, while the fit parameters do not depend significantly on the choice of the $Q^{2}$ cutoff, indicating that the dipole fits are stable.

We do the same comparison for $F_{2}^{u-d}\left(Q^{2}\right)$ as shown in Table XVII. Judging from the $\chi^{2} /$ dof values, we do not see significant differences between the dipole and tripole fits. Since the Pauli form factor is not constrained at $Q^{2}=0$, including larger $Q^{2}$ in the fits does not seem to affect the quality of the fits significantly. The fit parameters $F_{2}(0)$ and $M_{D, T}$ prove not to be affected as well.

As an example, we show the dipole fit curves with a $Q^{2}$ cutoff at $0.5,0.7$, and $1.1 \mathrm{GeV}^{2}$ for the $m_{\pi}=297 \mathrm{MeV}$ ensemble in the top panel of Fig. 4. To show the quality of the fits more clearly, we plot the ratios of the form factor data to the dipole fit with the $Q^{2}$ cutoff at $0.5 \mathrm{GeV}^{2}$ in the bottom three panels of Fig. 4. The error bands reflect the jackknife errors in the dipole fit parameters. We see that although the data included in the fits can be described reasonably well by the dipole formula with discrepancies that are generally within 2 to 3 standard deviations, the clear systematic tendency indicates that the dipole Ansatz is not a good description of the data over the whole momentum transfer region. In particular, for $F_{1}^{u-d}$, the precisely measured points in the region of $0.2 \mathrm{GeV}^{2}$ are systematically lower than the dipole fit, whereas at high $Q^{2}$, the lattice data are systematically higher. For $F_{2}^{u-d}$, the high $Q^{2}$ lattice data are systematically lower than the dipole fit. This is consistent with the empirical fits to the experimental data in Refs. [3,4], where the phenomenological corrections to the dipole form are negative in the region of $0.2 \mathrm{GeV}^{2}$ and positive at about $0.4 \mathrm{GeV}^{2}$. For comparison, we also plot the dipole fits with $Q^{2}$ cutoffs at $0.7 \mathrm{GeV}^{2}$ (dashed line) and $1.1 \mathrm{GeV}^{2}$ (dotted line) relative to the $0.5 \mathrm{GeV}^{2}$ dipole fit (solid line). The differences between different $Q^{2}$ cutoffs are small, indicating that the fits are stable.

It is worth noting that the Dirac and Pauli radii, $r_{1}^{v}$ and $r_{2}^{v}$, and the anomalous magnetic moment, $\kappa_{v}$, are defined in the $Q^{2}=0$ limit. We thus restrict the fits to the smallest $Q^{2}$ points possible to extract these quantities while still including enough data points to constrain the fits. For uniformity we choose to determine these quantities from the one-parameter dipole fits for $F_{1}^{u-d}$, and the two-parameter dipole fits for $F_{2}^{u-d}$, with a $Q^{2}$ cutoff at $0.5 \mathrm{GeV}^{2}$.

We also perform dipole fits to $G_{E}\left(Q^{2}\right)$ and $G_{M}\left(Q^{2}\right)$ to see how well the dipole Ansatz describes the data. We find that the dipole fits to $G_{E}^{u-d}$ and $G_{M}^{u-d}$ are qualitatively similar to $F_{1}^{u-d}$ and $F_{2}^{u-d}$. However, it appears that the fits are even more stable over the whole range of $Q^{2}$ than Dirac and Pauli form factors. This is indicated by little change in the ratio plots in Fig. 5 with different $Q^{2}$ cutoffs.

Figure 6 shows a comparison of the lattice results for $G_{E}$ at three different pion masses from the fine ensembles and one pion mass from the coarse ensemble with a phenomenological fit to the experimental data using the parameterization in Ref. [51] (with no indication of the experimental errors). The solid curves are dipole fits to the form factor results with the $Q^{2}$ cutoff at $0.5 \mathrm{GeV}^{2}$. As the pion mass decreases, the slope of the form factors at the small momentum transfer monotonically increases. The results from 

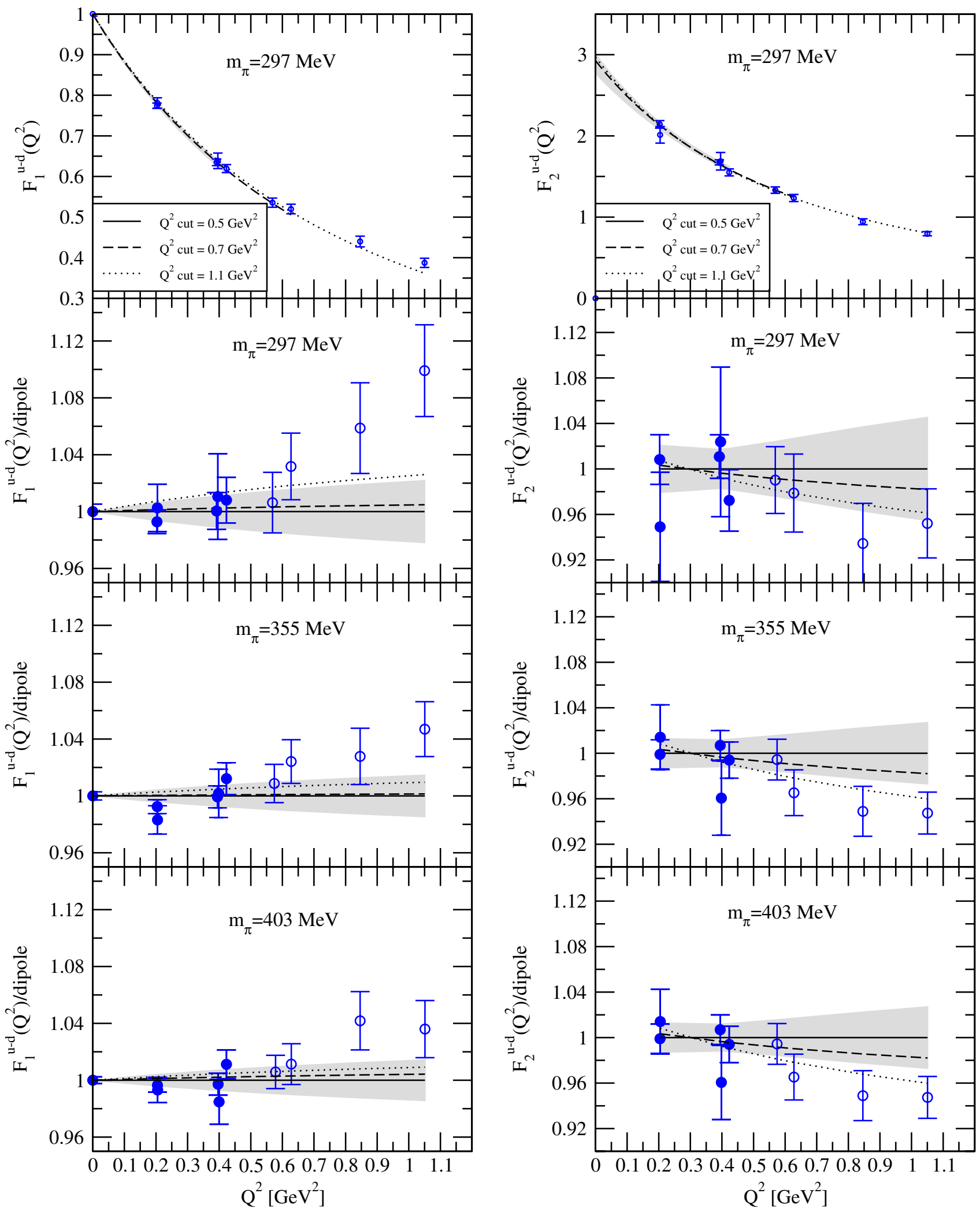

FIG. 4 (color online). The top panel shows the lattice results for $F_{1,2}^{u-d}\left(Q^{2}\right)$ at $m_{\pi}=297 \mathrm{MeV}$ along with the dipole fits with three different $Q^{2}$ cutoffs. The bottom left three panels show the ratios of the lattice results for $F_{1}^{u-d}$ to the dipole fits using Eq. (28), and the bottom right three panels show the ratios of the lattice results for $F_{2}^{u-d}$ to the dipole fits using Eq. (30). Only the solid data points are included in the fits with cutoff $0.5 \mathrm{GeV}^{2}$, and the grey bands show the errors for these fits. The dashed and dotted lines show the ratios of dipole fits at cutoffs $0.7 \mathrm{GeV}^{2}$ and $1.1 \mathrm{GeV}^{2}$ relative to the fit at $0.5 \mathrm{GeV}^{2}$. 

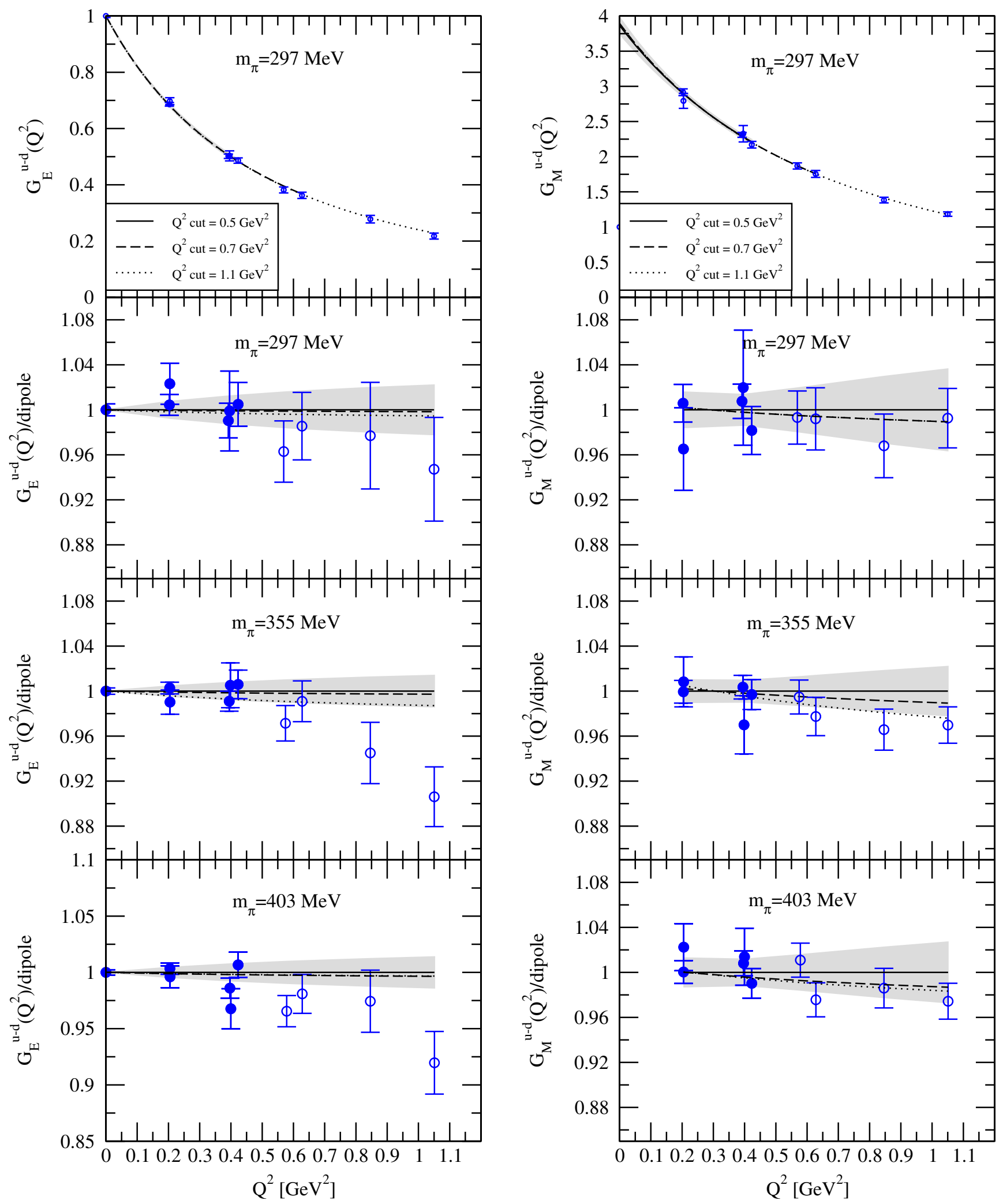

FIG. 5 (color online). The top panel shows the lattice results for $G_{E, M}^{u-d}\left(Q^{2}\right)$ at $m_{\pi}=297 \mathrm{MeV}$ along with the dipole fits with three different $Q^{2}$ cutoffs. The bottom left three panels show the ratios of the lattice results for $G_{E}^{u-d}$ to the dipole fits using Eq. (28), and the bottom right three panels show the ratios of the lattice results for $G_{M}^{u-d}$ to the dipole fits using Eq. (30). Only the solid data points are included in the fits with cutoff $0.5 \mathrm{GeV}^{2}$, and the grey bands show the errors for these fits. The dashed and dotted lines show the ratios of dipole fits at cutoffs $0.7 \mathrm{GeV}^{2}$ and $1.1 \mathrm{GeV}^{2}$ relative to the fit at $0.5 \mathrm{GeV}^{2}$. 


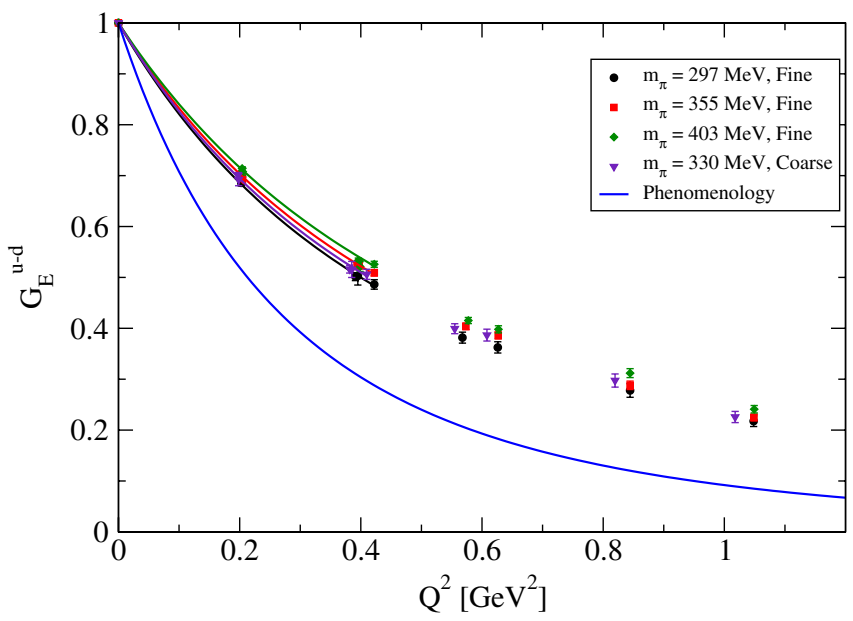

the coarse ensemble at $m_{\pi}=330 \mathrm{MeV}$ is nicely surrounded by the results from the fine ensembles at $m_{\pi}=$ 297 and $m_{\pi}=355 \mathrm{MeV}$, indicating that the effect of the finite lattice spacing error should be small.

\section{Chiral extrapolations}

\section{Chiral extrapolations using $\mathcal{O}\left(\epsilon^{3}\right)$ small scale expansion}

To compare the lattice results for the nucleon form factors at finite momentum transfer with the experimental
FIG. 6 (color online). Lattice results for $G_{E}^{u-d}$ at three pion masses from the fine ensembles and one pion mass from the coarse ensemble, compared with a phenomenological fit to the experimental data as parameterized in Ref. [51]. The solid curves are the dipole fits to the form factor results with a cutoff at $Q^{2}=$ $0.5 \mathrm{GeV}^{2}$.

$$
\begin{aligned}
F_{1}^{u-d}\left(Q^{2}, m_{\pi}\right)= & 1+\frac{1}{\left(4 \pi F_{\pi}\right)^{2}}\left\{-Q^{2}\left(\frac{68}{81} c_{A}^{2}-\frac{2}{3} g_{A}^{2}-2 B_{10}^{(r)}(\lambda)\right)-Q^{2}\left(\frac{40}{27} c_{A}^{2}-\frac{5}{3} g_{A}^{2}-\frac{1}{3}\right) \log \left[\frac{m_{\pi}}{\lambda}\right]\right. \\
& +\int_{0}^{1} d x\left[\frac{16}{3} \Delta^{2} c_{A}^{2}+m_{\pi}^{2}\left(3 g_{A}^{2}+1-\frac{8}{3} c_{A}^{2}\right)+Q^{2} x(1-x)\left(5 g_{A}^{2}+1-\frac{40}{9} c_{A}^{2}\right)\right] \log \left[\frac{\tilde{m}^{2}}{m_{\pi}^{2}}\right] \\
& +\int_{0}^{1} d x\left[-\frac{32}{9} c_{A}^{2} Q^{2} x(1-x) \frac{\Delta \log R(\tilde{m})}{\sqrt{\Delta^{2}-\tilde{m}^{2}}}\right] \\
& \left.-\int_{0}^{1} d x \frac{32}{3} c_{A}^{2} \Delta\left[\sqrt{\Delta^{2}-m_{\pi}^{2}} \log R\left(m_{\pi}\right)-\sqrt{\Delta^{2}-\tilde{m}^{2}} \log R(\tilde{m})\right]\right\},
\end{aligned}
$$

where

$$
\begin{aligned}
& R(m)=\frac{\Delta}{m}+\sqrt{\frac{\Delta^{2}}{m^{2}}-1,} \\
& \tilde{m}^{2}=m_{\pi}^{2}+Q^{2} x(1-x) .
\end{aligned}
$$

In the above expressions, $F_{\pi}$ denotes the pion decay constant in the $S U(2)$ chiral limit with the convention in results, we need to do extrapolations for both the $m_{\pi}$ and $Q^{2}$ dependence using baryon chiral perturbation theory. This combined dependence has been worked out both in SSE at leading one-loop accuracy and in BChPT up to next-to-next-to-leading-order (NNLO). In particular, the $\mathcal{O}\left(\epsilon^{3}\right)$ expression for the isovector Dirac form factor $F_{1}^{u-d}\left(Q^{2}, m_{\pi}\right)$ has been derived in Ref. [44] and is given by

$$
\begin{aligned}
F_{2}^{u-d}\left(Q^{2}, m_{\pi}\right)= & \kappa_{v}\left(m_{\pi}\right)-g_{A}^{2} \frac{4 \pi M_{N}}{\left(4 \pi F_{\pi}\right)^{2}} \int_{0}^{1} d x\left[\sqrt{\tilde{m}^{2}}-m_{\pi}\right]+\frac{32 c_{A}^{2} M_{N} \Delta}{9\left(4 \pi F_{\pi}\right)^{2}} \int_{0}^{1} d x\left[\frac{1}{2} \log \left[\frac{\tilde{m}^{2}}{4 \Delta^{2}}\right]-\log \left[\frac{m_{\pi}}{2 \Delta}\right]\right. \\
& \left.+\frac{\sqrt{\Delta^{2}-\tilde{m}^{2}}}{\Delta} \log R(\tilde{m})-\frac{\sqrt{\Delta^{2}-m_{\pi}^{2}}}{\Delta} \log R\left(m_{\pi}\right)\right]
\end{aligned}
$$

\footnotetext{
${ }^{6}$ The coupling $c_{A}$ corresponds to $\dot{g}_{\pi N \Delta}$ in the notation of Ref. [31].
} 
where, to $\mathcal{O}\left(\epsilon^{3}\right)$,

$$
\kappa_{v}\left(m_{\pi}\right)=\kappa_{v}^{0}-\frac{g_{A}^{2} m_{\pi} M_{N}}{4 \pi F_{\pi}^{2}}+\frac{2 c_{A}^{2} \Delta M_{N}}{9 \pi^{2} F_{\pi}^{2}}\left\{\sqrt{1-\frac{m_{\pi}^{2}}{\Delta^{2}}} \log \left[R\left(m_{\pi}\right)\right]+\log \left[\frac{m_{\pi}}{2 \Delta}\right]\right\}+\mathcal{O}\left(m_{\pi}^{2}\right)
$$

In order to capture the most prominent $\mathcal{O}\left(m_{\pi}^{2}\right)$ corrections, Hemmert and Weise [52] proposed a modification of the standard SSE power counting to promote the leading term of the magnetic $N \rightarrow \Delta$ transition into the first order $N \Delta$ effective Lagrangian. This leads to the following expression for $\kappa_{v}\left(m_{\pi}\right)$ :

$$
\begin{aligned}
\kappa_{v}\left(m_{\pi}\right)= & \kappa_{v}^{0}-\frac{g_{A}^{2} m_{\pi} M_{N}}{4 \pi F_{\pi}^{2}}+\frac{2 c_{A}^{2} \Delta M_{N}}{9 \pi^{2} F_{\pi}^{2}}\left\{\sqrt{1-\frac{m_{\pi}^{2}}{\Delta^{2}}} \log \left[R\left(m_{\pi}\right)\right]+\log \left[\frac{m_{\pi}}{2 \Delta}\right]\right\}-8 E_{1}^{r}(\lambda) M_{N} m_{\pi}^{2}+\frac{4 c_{A} c_{V} g_{A} M_{N} m_{\pi}^{2}}{9 \pi^{2} F_{\pi}^{2}} \\
& \times \log \left[\frac{2 \Delta}{\lambda}\right]+\frac{4 c_{A} c_{V} g_{A} M_{N} m_{\pi}^{3}}{27 \pi F_{\pi}^{2} \Delta}-\frac{8 c_{A} c_{V} g_{A} \Delta^{2} M_{N}}{27 \pi^{2} F_{\pi}^{2}}\left\{\left(1-\frac{m_{\pi}^{2}}{\Delta^{2}}\right)^{3 / 2} \log \left[R\left(m_{\pi}\right)\right]+\left(1-\frac{3 m_{\pi}^{2}}{2 \Delta^{2}}\right) \log \left[\frac{m_{\pi}}{2 \Delta}\right]\right\},
\end{aligned}
$$

where $c_{V}$ is the leading magnetic photon-nucleon- $\Delta$ coupling in the chiral limit and $\kappa_{v}^{0}$ denotes the anomalous magnetic moment in the $S U(2)$ chiral limit. We will use this expression in our analysis.

Our results for the form factor $F_{2}$ are given in terms of a quark mass-dependent "magneton" [see Eq. (24)], which is not accounted for in SSE at the order at which we are working. Therefore, in order to compare Eq. (40) with our lattice data, we follow Refs. [16,17] and define $\kappa^{\text {norm }}$ measured relative to the physical magneton:

$$
\kappa^{\text {norm }}=\frac{M_{N}^{\text {phys }}}{M_{N}^{\text {lat }}} \kappa^{\text {lat }}=\frac{M_{N}^{\text {phys }}}{M_{N}^{\text {lat }}} F_{2}(0)
$$

We then identify $M_{N}$ in the SSE expressions as the physical nucleon mass. In the following comparisons of our results with chiral perturbation theories, the normalized magnetic moment $\kappa_{v}^{\text {norm }}$ will be used throughout, and we drop the superscript "norm" unless there is an ambiguity.

ChPT describes the $Q^{2}$ dependence of the form factors for values of $Q^{2}$ much less than the chiral symmetry breaking scale (typically of the order of the nucleon mass) and $Q^{2}$ counts as a small quantity, of the order of $m_{\pi}^{2}$. In fact, we have attempted simultaneous fits to both the $m_{\pi}$ and $Q^{2}$ dependences of $F_{1}^{u-d}$ using the SSE formula in Eq. (35), and found that the fits fail to describe data even with $Q^{2} \leq$ $0.4 \mathrm{GeV}^{2}\left(\chi^{2} /\right.$ dof $\left.\approx 10\right)$. This is consistent with the findings of Ref. [44], where the applicability of the $\mathcal{O}\left(\epsilon^{3}\right)$ SSE results for the isovector nucleon form factors at physical pion mass was found to be limited to $Q^{2}<0.2 \mathrm{GeV}^{2}$. Lacking a model-independent functional form applicable in the large- $Q^{2}$ region, we resort to studying the pion-mass dependence of the mean squared Dirac radius, $\left(r_{1}^{v}\right)^{2}$, Pauli radius, $\left(r_{2}^{v}\right)^{2}$, and the anomalous magnetic moment, $\kappa_{v}$, as obtained from the dipole fits discussed in Sec. III B. We tabulate these values in Table IV.

The $\mathcal{O}\left(\epsilon^{3}\right)$ SSE formulas for $\left(r_{1}^{v}\right)^{2}$ and $\left(r_{2}^{v}\right)^{2}$ can be derived from Eqs. (35) and (38), respectively, and are given by

$$
\begin{aligned}
\left(r_{1}^{v}\right)^{2}= & -\frac{1}{\left(4 \pi F_{\pi}\right)^{2}}\left\{1+7 g_{A}^{2}+\left(10 g_{A}^{2}+2\right) \log \left[\frac{m_{\pi}}{\lambda}\right]\right\} \\
& -\frac{12 B_{10}^{(r)}(\lambda)}{\left(4 \pi F_{\pi}\right)^{2}}+\frac{c_{A}^{2}}{54 \pi^{2} F_{\pi}^{2}}\left\{26+30 \log \left[\frac{m_{\pi}}{\lambda}\right]\right. \\
& +30 \frac{\Delta}{\sqrt{\Delta^{2}-m_{\pi}^{2}}} \log \left[\frac{\Delta}{m_{\pi}}+\sqrt{\left.\left.\frac{\Delta^{2}}{m_{\pi}^{2}}-1\right]\right\}+\mathcal{O}\left(m_{\pi}\right),}\right.
\end{aligned}
$$

$$
\begin{aligned}
\kappa_{v}\left(m_{\pi}\right) \cdot\left(r_{2}^{v}\right)^{2}= & \frac{g_{A}^{2} M_{N}}{8 \pi F_{\pi}^{2} m_{\pi}}+\frac{c_{A}^{2} M_{N}}{9 \pi^{2} F_{\pi}^{2} \sqrt{\Delta^{2}-m_{\pi}^{2}}} \\
& \times \log \left[\frac{\Delta}{m_{\pi}}+\sqrt{\frac{\Delta^{2}}{m_{\pi}^{2}}-1}\right]+\mathcal{O}\left(m_{\pi}^{0}\right) .
\end{aligned}
$$

TABLE IV. Results for the isovector Dirac and Pauli radii and anomalous magnetic moment from dipole fits with $Q^{2} \leq$ $0.5 \mathrm{GeV}^{2}$.

\begin{tabular}{l|cccc}
\hline \hline $\begin{array}{l}m_{\pi} \\
{[\mathrm{MeV}]}\end{array}$ & $\begin{array}{c}\left(r_{1}^{v}\right)^{2} \\
{\left[\mathrm{fm}^{2}\right]}\end{array}$ & $\begin{array}{c}\left(r_{2}^{v}\right)^{2} \\
{\left[\mathrm{fm}^{2}\right]}\end{array}$ & $\begin{array}{c}\kappa_{v}^{\text {norm }} \cdot\left(r_{2}^{v}\right)^{2} \\
{\left[\mathrm{fm}^{2}\right]}\end{array}$ & $\kappa_{v}^{\text {norm }}$ \\
\hline 297 & $0.305(8)$ & $0.382(33)$ & $0.938(117)$ & $2.447(99)$ \\
355 & $0.281(5)$ & $0.372(18)$ & $0.938(66)$ & $2.518(57)$ \\
403 & $0.272(5)$ & $0.379(16)$ & $0.954(58)$ & $2.508(51)$ \\
330 & $0.290(9)$ & $0.445(26)$ & $1.230(105)$ & $2.758(84)$ \\
\hline \hline
\end{tabular}


Together with the expression for the anomalous magnetic moment in Eq. (40), these formulas involve six lowenergy constants: $F_{\pi}, \Delta, c_{A}, g_{A}, \kappa_{v}^{0}$, and $c_{V}$, as well as two counterterms: $B_{10}^{r}(\lambda)$ and $E_{1}^{r}(\lambda)$. Ideally we would like to determine all these constants from simultaneous fits to lattice results. However, this is not feasible with the limited number of measured observables and pion masses in the present calculation, and we thus fix some of the low-energy constants using their phenomenological values. We describe our choices for these values below.

In Ref. [53], Colangelo and Dürr analyze numerically the NNLO expression for the pion-mass dependence of $F_{\pi}$ [48]. They use available information from phenomenology to fix all low-energy constants but the chiral limit value of $F_{\pi}$, use the physical value (11) and obtain

$$
\left.F_{\pi}\right|_{\text {chiral limit }}=(86.2 \pm 0.5) \mathrm{MeV} \text {. }
$$

In the absence of reliable chiral extrapolations of both nucleon and $\Delta(1232)$ masses (see the discussion in Ref. [54]), ${ }^{7}$ we identify the $\Delta$-nucleon mass splitting in the chiral limit with its value at the physical $m_{\pi}$. The position of the $\Delta(1232)$ resonance pole in the total center-of-mass energy plane has been determined from magnetic dipole and electric quadrupole amplitudes of pion photoproduction. According to the Particle Data Group average [56], the $\Delta$-pole position leads to $M_{\Delta}=$ $(1210 \pm 1) \mathrm{MeV}$ and $\Gamma_{\Delta}=(100 \pm 2) \mathrm{MeV}$. If one instead defines the $\Delta(1232)$ mass and width by looking at the $90^{\circ}$ $\pi N$ phase shift in the spin-3/2 isospin-3/2 channel, the PDG averages give $M_{\Delta}=(1232 \pm 1) \mathrm{MeV}$ and $\Gamma_{\Delta}=$ $(118 \pm 2) \mathrm{MeV}$. With $M_{N}=939 \mathrm{MeV}$, one obtains, respectively,

$$
\Delta=(271 \pm 1) \mathrm{MeV}
$$

or

$$
\Delta=(293 \pm 1) \mathrm{MeV} .
$$

The $\Delta(1232)$ decays strongly to a nucleon and a pion with almost $100 \%$ branching fraction. From the PDG values of masses and widths [56] and from

$$
\Gamma_{\Delta \rightarrow N \pi}=\frac{c_{A}^{2}}{12 \pi F_{\pi}^{2} M_{\Delta}}\left(E_{\pi}^{2}-m_{\pi}^{2}\right)^{3 / 2}\left(M_{\Delta}+M_{N}-E_{\pi}\right),
$$

where

$$
E_{\pi}=\frac{M_{\Delta}^{2}-M_{N}^{2}+m_{\pi}^{2}}{2 M_{\Delta}}
$$

\footnotetext{
${ }^{7}$ For an analysis of the quark-mass dependence of nucleon and delta masses in the covariant SSE at order $\epsilon^{4}$ we refer to [55].
}

one obtains, respectively, ${ }^{8}$

$$
\begin{aligned}
& \left|c_{A}\right|=1.50 \ldots 1.55 \text { if } \Gamma=(100 \pm 2) \mathrm{MeV} \text { and } \\
& \Delta=(271 \pm 1) \mathrm{MeV} \\
& \left|c_{A}\right|=1.43 \ldots 1.47 \text { if } \Gamma=(118 \pm 2) \mathrm{MeV} \\
& \quad \text { and } \Delta=(293 \pm 1) \mathrm{MeV} .
\end{aligned}
$$

Chiral extrapolations of different sets of lattice results [57-60] based on SSE at leading one-loop accuracy lead to a chiral limit value for $g_{A}$ of about 1.2. From the relativistic tree-level analysis of the process of pion photoproduction at threshold $\gamma p \rightarrow \pi^{0} p$, one obtains [61,62] (for $c_{A}=1.5$ )

$$
c_{V}=(-2.5 \pm 0.4) \mathrm{GeV}^{-1} .
$$

As specified above, at the order $\mathcal{O}\left(\epsilon^{3}\right)$, all the couplings in Eqs. (35)-(43) are meant to be taken in the chiral limit. Replacing them with the corresponding quantities at the physical point amounts to the inclusion of higher-order effects. As long as the deviation between the values in the chiral limit and at the physical point is small, one expects such a replacement to yield little effect. To test this statement, in some cases we have performed the chiral fits using both the physical values and the chiral limit values for the low-energy constants and found no significant differences. In the following we will only present results obtained using the chiral limit values as inputs, which are summarized in Table V.

Among the low-energy constants discussed above, $c_{A}$ and $c_{V}$ are the two least known. In addition, we have little knowledge of the counterterms, $B_{10}^{r}(\lambda)$ and $E_{1}^{r}(\lambda)$, as well as the anomalous magnetic moment in the chiral limit, $\kappa_{v}^{0}$, from phenomenology. Lattice calculations in the chiral

${ }^{8}$ Calculating the strong decay width of $\Delta(1232)$ to leading order in (nonrelativistic) SSE kinematics, one obtains

$$
\Gamma_{\Delta \rightarrow N \pi}=\frac{c_{A}^{2}}{6 \pi F_{\pi}^{2}}\left(\Delta^{2}-m_{\pi}^{2}\right)^{3 / 2} .
$$

We note that this expression corresponds to the leading term in a $1 / M_{N}$ expansion of the result given in Eq. (47), which utilizes the full covariant kinematics. Using the ranges of masses and decay widths mentioned above, this expression yields the lower values

$$
\begin{aligned}
\left|c_{A}\right| & =1.11 \ldots 1.14 \quad \text { if } \Gamma=(100 \pm 2) \mathrm{MeV} \text { and } \\
\Delta & =(271 \pm 1) \mathrm{MeV} ;
\end{aligned}
$$

$$
\begin{aligned}
\left|c_{A}\right| & =1.04 \ldots 1.07 \quad \text { if } \Gamma=(118 \pm 2) \mathrm{MeV} \text { and } \\
\Delta & =(293 \pm 1) \mathrm{MeV} .
\end{aligned}
$$

Furthermore, $S U(4)$ spin-flavor quark symmetry gives $c_{A}=$ $3 g_{A} /(2 \sqrt{2})=1.34$. 
TABLE V. Input values for the low-energy constants in the fits.

\begin{tabular}{lcc}
\hline \hline$g_{A}$ & $F_{\pi}[\mathrm{GeV}]$ & $\Delta[\mathrm{GeV}]$ \\
\hline 1.2 & 0.0862 & 0.293 \\
\hline \hline
\end{tabular}

regime have the potential to constrain these parameters to unprecedented accuracy. Our attempt here is to check the consistency of our data with the predictions of chiral effective field theories, to estimate the range of applicability of the ChPT formulas, and to determine these lowenergy constants when the formulas are applicable. To disentangle the investigation of the applicability of the ChPT formula from the possible discretization effects, we only include the results from the three fine ensembles in the chiral extrapolations discussed below. However, we want to point out that including the coarse results in the extrapolations does not change the central values of the fit parameters significantly, nor does it reduce the errors on the parameters since it only adds an additional interpolating point and does not provide a much stronger constraint on the parameters.

Since $c_{A}$ appears in the formulas for $\left(r_{1}^{v}\right)^{2},\left(r_{2}^{v}\right)^{2}$, and $\kappa_{v}$, a simultaneous fit to all these three quantities would give a better constraint for the value of $c_{A}$. However, we have only three data points for each of these quantities, and $\kappa_{v}$ alone has four parameters, three of which $\left[c_{V}, E_{1}^{r}(\lambda)\right.$, and $\left.\kappa_{v}^{0}\right]$ are not constrained by any other quantity. Thus the quark-mass dependence of $\kappa_{v}$ cannot be used to constrain $c_{A}$. Therefore we choose to fit simultaneously ${ }^{9}$ only $\left(r_{1}^{v}\right)^{2}$ and $\kappa_{v} \cdot\left(r_{2}^{v}\right)^{2}$ to determine $c_{A}$ and $B_{10}^{r}(\lambda)$, and then use the resulting $c_{A}$ as an input for the fit to $\kappa_{v}$. This way the three free parameters in $\kappa_{v}$ are exactly specified by the three data points.

We present the resulting $\chi^{2} /$ dof and fit parameters normalized at scale $\lambda=600 \mathrm{MeV}$ in the first row of Table VI and plot the fit curves as the solid lines in Fig. 7. As indicated by a $\chi^{2} /$ dof of 17 , the simultaneous fit to $\left(r_{1}^{v}\right)^{2}$ and $\kappa_{v} \cdot\left(r_{2}^{v}\right)^{2}$ does not describe the data. The problem is that our results for $\left(r_{1}^{v}\right)^{2}$ and $\kappa_{v} \cdot\left(r_{2}^{v}\right)^{2}$ favor different values for $c_{A}$. In fact, an independent fit to $\left(r_{1}^{v}\right)^{2}$ yields $c_{A}=1.98(7)$, while an independent fit to $\kappa_{v} \cdot\left(r_{2}^{v}\right)^{2}$ gives $c_{A}=1.39(10)$. The tension between these two quan-

\footnotetext{
${ }^{9}$ We note however, that in Ref. [16] it was already observed that the leading one-loop SSE formula for $\left(r_{1}^{v}\right)^{2}$ [Eq. (42)] is dominated by the leading chiral logarithm and dropped below the level of the lattice data available at that time for values of the pion mass as low as $m_{\pi}<200 \mathrm{MeV}$. This prompted the authors of Ref. [16] to exclude the isovector Dirac radius from the simultaneous fit. Likewise, the authors of Ref. [17] obtained huge, unrealistic values for the isovector Dirac radius when trying to enforce a fit of the logarithm-dominated behavior onto their data. Given these two negative precedents, we consider our "fit" to the isovector Dirac radius data to be of exploratory nature, testing the limits of applicability of the leading one-loop SSE results given in Eq. (42).
}

tities results in the large $\chi^{2} /$ dof in the simultaneous fit, indicating that the formulas given in Eqs. (42) and (43) do not describe our data consistently. As we can see from Fig. 7(b), the solid fit curve lies systematically higher than the data points, which then motivates us to add the $\mathcal{O}\left(m_{\pi}^{0}\right)$ correction to the leading one-loop result of Eq. (43) (the socalled "core" contribution in Ref. [16]) to $\kappa_{v} \cdot\left(r_{2}^{v}\right)^{2}$, such that

$$
\begin{aligned}
\kappa_{v}\left(m_{\pi}\right) \cdot\left(r_{2}^{v}\right)^{2}= & \frac{g_{A}^{2} M_{N}}{8 \pi F_{\pi}^{2} m_{\pi}}+\frac{c_{A}^{2} M_{N}}{9 \pi^{2} F_{\pi}^{2} \sqrt{\Delta^{2}-m_{\pi}^{2}}} \\
& \times \log \left[\frac{\Delta}{m_{\pi}}+\sqrt{\frac{\Delta^{2}}{m_{\pi}^{2}}-1}\right]+24 M_{N} \mathcal{C} .
\end{aligned}
$$

With this modification, the simultaneous fit to $\left(r_{1}^{v}\right)^{2}$ and $\kappa_{v} \cdot\left(r_{2}^{v}\right)^{2}$, now using Eqs. (42) and (55), appears to describe the average value of the data much better, but still not the pion-mass dependence. We show the results in the second row of Table VI, and the fit curves (dashed lines) in Fig. 7. The fit describes $\left(r_{1}^{v}\right)^{2}$ very well, but $c_{A}$ turns out to be larger than the range discussed earlier, which, not surprisingly, gives rise to a smaller extrapolated value for $\left(r_{1}^{v}\right)^{2}$ than the experiments. Our new DWF data extend the trend of the weak pion-mass dependence in $\left(r_{2}^{v}\right)^{2}$ observed in Refs. [16,17] now down into the range of pion masses $\sim 300 \mathrm{MeV}$. The appearance of such a "plateaulike" behavior down to such light pion masses, which was also observed in Ref. [29], is surprising. The leading one-loop SSE formulas (43) and (55) for this radius cannot accommodate such a behavior, with or without the inclusion of the higher-order core term.

Using $c_{A}$ determined from the above fits either with or without the constant term in Eq. (43) to $\left(r_{1}^{v}\right)^{2}$ and $\kappa_{v}$. $\left(r_{2}^{v}\right)^{2}$, we fit $\kappa_{v}$ to Eq. (40) with three unknown parameters, $\kappa_{v}^{0}, c_{V}$, and $E_{1}^{r}(\lambda)$. The results are shown in Table VI. The value for $c_{V}$ from our fit turns out to have a different sign from that determined in $[61,62]$ mentioned earlier. This is not surprising given that we only have three data points, which have little or no pion-mass dependence. We do not have the freedom to check the consistency of the fit, and we do not expect to obtain a reliable estimation for $c_{V}$, which, judging from Eq. (40), is very sensitive to the curvature of the data.

To compare chiral extrapolations with experiment, we have also plotted selected experimental data in Fig. 7. As noted in the introduction, there are still unresolved experimental questions, and we have indicated the range of possible values of $\left(r_{1}^{v}\right)^{2}$ that can be extracted from present experiments by showing two extreme results from the literature. The highest value is from PDG 2008 [56] and the lowest value is from a dispersion analysis including meson continuum contributions [7]. We note that none of the chiral fits simultaneously yields a good fit to the lattice 
TABLE VI. Fit parameters from the fits to the isovector Dirac radius $\left(r_{1}^{v}\right)^{2}$, Pauli radius $\left(r_{2}^{v}\right)^{2}$ and the anomalous magnetic moment $\kappa_{v}$. Details of the fit procedures are described in the text. We have set the scale to $\lambda=600 \mathrm{MeV}$.

\begin{tabular}{l|rcccccc}
\hline \hline & \multicolumn{1}{|c}{$\chi^{2} /$ dof } & $c_{A}$ & $c_{V}\left[\mathrm{GeV}^{-1}\right]$ & $\kappa_{v}^{0}$ & $B_{10}^{r}(\lambda)$ & $E_{1}^{r}(\lambda)\left[\mathrm{GeV}^{-3}\right]$ & $\mathcal{C}\left[\mathrm{GeV}^{-3}\right]$ \\
\hline No constant term & $17.0(4.0)$ & $1.54(6)$ & $8.7(5.8)$ & $4.13(95)$ & $1.20(17)$ & $-4.67(42)$ & \\
With constant term & $3.8(2.2)$ & $1.97(7)$ & $7.5(4.5)$ & $4.32(95)$ & $2.58(25)$ & $-5.58(42)$ & $-0.51(7)$ \\
\hline \hline
\end{tabular}

data while also agreeing with experiment within statistical errors.

To see how strongly the lattice results deviate from the SSE formulas, we also try to determine some of the lowenergy constants using experimental results at the physical pion mass. We use the values in Table $\mathrm{V}$ as input, and also set $c_{A}=1.5$ and $c_{V}=-2.5 \mathrm{GeV}^{-1}$. Now for $\left(r_{1}^{v}\right)^{2}$, we have only the counterterm $B_{10}^{r}$ to determine. Constraining the curve to go through the higher experimental value of $\left(r_{1}^{v}\right)^{2}=0.637 \mathrm{fm}^{2}$ gives $B_{10}^{r}(\lambda=600 \mathrm{MeV})=1.085$, resulting in the solid curve shown in Fig. 8(a). For compari- son, we also plot the dashed curve that is fixed to go through the lower experimental value $\left(r_{1}^{v}\right)^{2}$. The curve rises much more rapidly than the lattice data as the pion mass decreases. From the slope of the leading one-loop SSE curve near the physical point and the weak pion-mass dependence displayed by our data we estimate that the applicability of Eq. (42) for $\left(r_{1}^{v}\right)^{2}$ may be much less than $300 \mathrm{MeV}$.

Without the constant term in Eq. (55), $\kappa_{v} \cdot\left(r_{2}^{v}\right)^{2}$ does not have any free parameters, which yields the solid curve in Fig. 8(b). The curve undershoots the physical point by

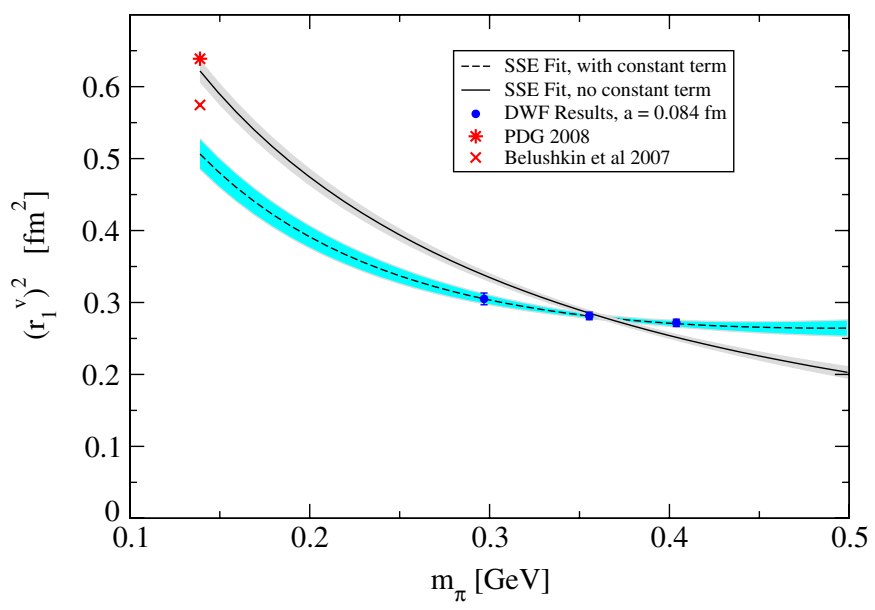

(a)

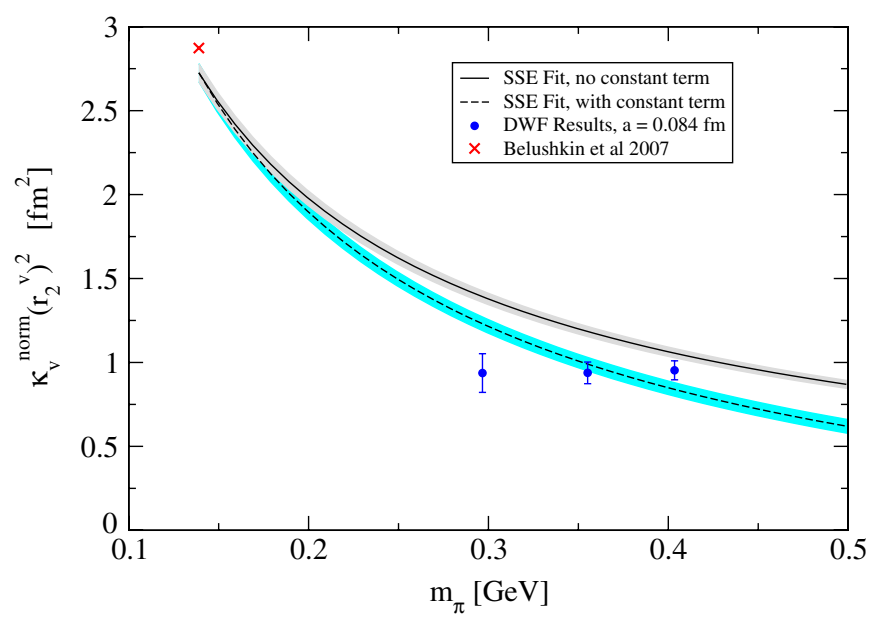

(b)

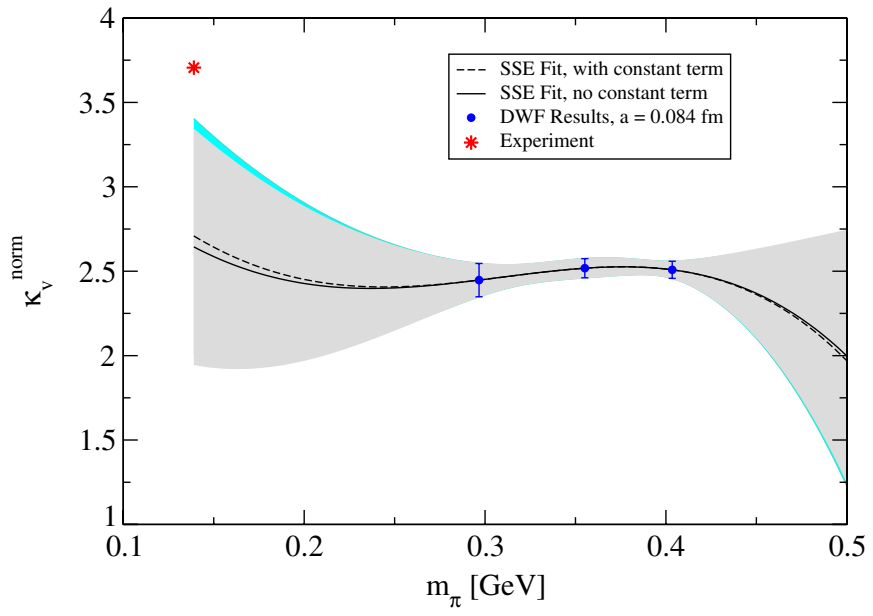

(c)

FIG. 7 (color online). Chiral extrapolations for the isovector Dirac radius $\left(r_{1}^{v}\right)^{2}$ (a), Pauli radius $\kappa_{v} \cdot\left(r_{2}^{v}\right)^{2}$ (b), and the anomalous magnetic moment $\kappa_{v}$ (c) using the $\mathcal{O}\left(\epsilon^{3}\right)$ SSE formula with (solid curves) or without (dashed curves) the constant term in Eq. (55). In both cases, $\left(r_{1}^{v}\right)^{2}$ and $\kappa_{v} \cdot\left(r_{2}^{v}\right)^{2}$ are fit simultaneously, while $\kappa_{v}$ is fit separately with $c_{A}$ determined from the simultaneous fit. 


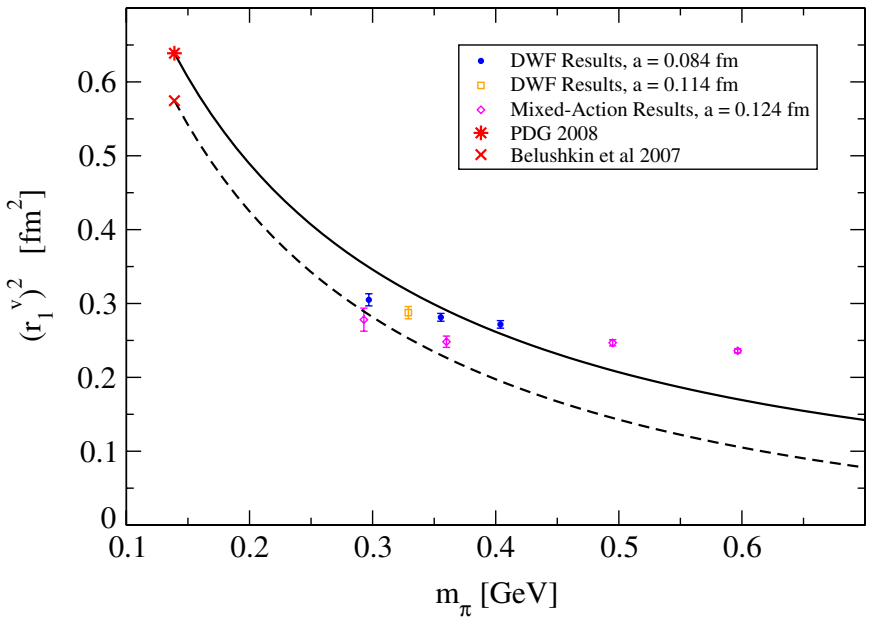

(a)

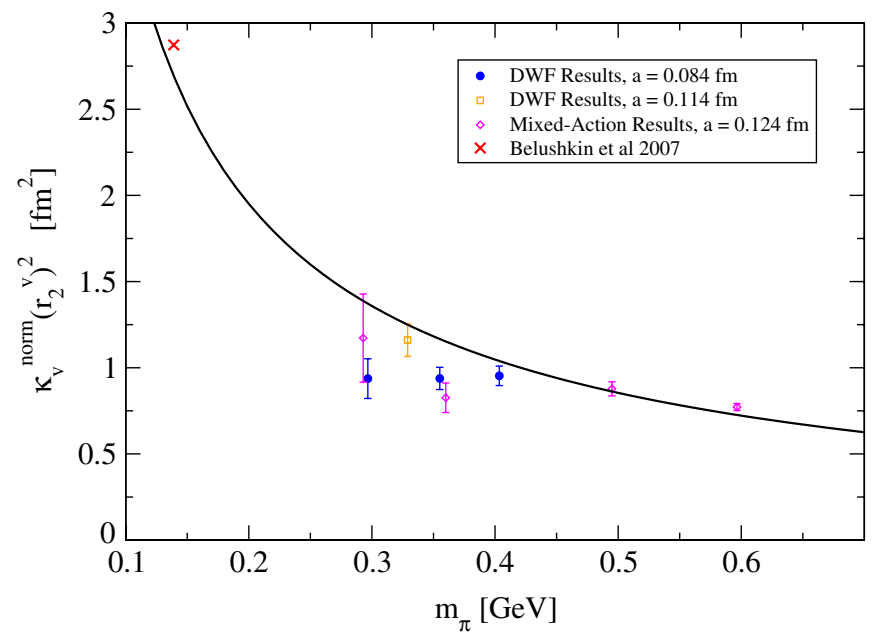

(b)

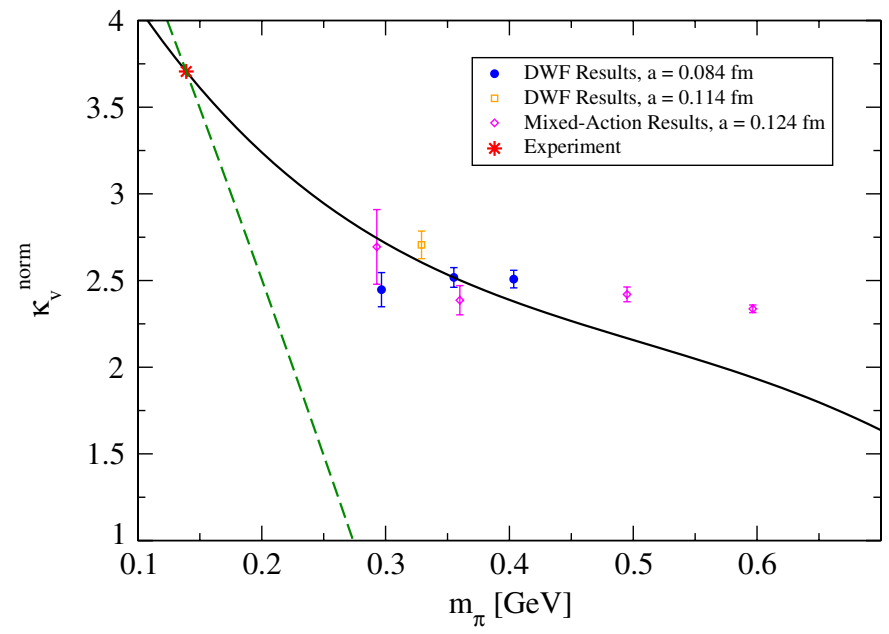

(c)

FIG. 8 (color online). SSE chiral fits to the isovector Dirac radius $\left(r_{1}^{v}\right)^{2}$ (a), Pauli radius $\kappa_{v} \cdot\left(r_{2}^{v}\right)^{2}$ (b), and the anomalous magnetic moment $\kappa_{v}$ (c) constrained to go through the physical points using the input in Table $\mathrm{V}$ as well as $c_{A}=1.5$ and $c_{V}=-2.5 \mathrm{GeV}^{-1}$. The mixed-action results at $m_{\pi}=355 \mathrm{MeV}$ are shifted slightly to the right for clarity. In (a) the solid curve is constrained to go through the physical result given in PDG 2008, and the dashed curve is constrained to go through the result given in Ref. [7]. In (b) the curve is drawn using the input low-energy constants according to Eq. (43). In (c) the solid curve is constrained to go through the physical point as well as our DWF result at $m_{\pi}=355 \mathrm{MeV}$ using Eq. (40), while the dashed curve is constrained to go through the physical point using Eq. (39).

about $5 \%$, which may be well accounted for by the uncertainties in the chosen values of the low-energy constants. Including the higher-order term $\mathcal{C}$ of Eq. (55) can of course shift the curve up to exactly reproduce the product of physical Pauli radius and anomalous magnetic moment. However, the departure of the quark-mass dependent curve from the lattice data displayed in Fig. 8(b) indicates that the leading one-loop SSE formula for $\kappa_{v}$. $\left(r_{2}^{v}\right)^{2}$ of Eqs. (43) and (55) should only be trusted for pion masses much less than the currently available $300 \mathrm{MeV}$. Judging from the steep slopes displayed by both the curves for the Dirac and Pauli radii as opposed to the almost massindependent nature of the lattice data, it is conceivable that the leading one-loop SSE formulas may only be applicable at pion masses well below $300 \mathrm{MeV}$, as already suggested in Ref. [16].

The anomalous magnetic moment still has two free parameters, $E_{1}^{r}$ and $\kappa_{v}^{0}$. In addition to the physical point, we need another data point to determine both parameters. We choose to use our $m_{\pi}=355 \mathrm{MeV}$ result in the determination, since this point is the most accurately calculated and its relatively large pion mass makes it less susceptible to finite-volume effects. The resulting curve (the solid line) is given in Fig. 8(c). For comparison, we also show the curve using the leading-order SSE formula in Eq. (39) (the dashed line). In this case, only the experimental point is included to determine $\kappa_{v}^{0}$. We can see that the dashed line deviates greatly from the lattice data. This is not surprising, 
as the dominating contribution to $\kappa_{v}$ is the term linear in $m_{\pi}$, the coefficient of which is determined by $\frac{g_{A}^{2} M_{N}}{4 \pi F_{\pi}^{2}}$. This is clearly not the case in our data. Regarding the limit of applicability of Eq. (40) [which includes the dominant next-to-leading one-loop corrections to the strict $\mathcal{O}\left(\epsilon^{3}\right)$ SSE result of Eq. (39)], the plot in Fig. 8(c) does not give us a clear indication up to which pion mass the formula can be quantitatively employed. Furthermore, we observe that the "normalized" anomalous magnetic moments display a flat pion-mass dependence around 2.5 nuclear magnetons. The new dynamical DWF data extend this "plateau" of the normalized magnetic momentswhich was already observed at much larger pion masses in the quenched simulation of Ref. [16] — now into the region of pion masses as low as $300 \mathrm{MeV}$. Surprisingly, we can find no indication of a rise in the magnetic moment at these low pion masses, although the onset of such a rise had been anticipated for pion masses around $300 \mathrm{MeV}$ in the fit results of Ref. [16] (see Fig. 11).

Overall, these curves show much stronger curvatures than our lattice results. Even with pion masses as light as $300 \mathrm{MeV}$, the $\mathcal{O}\left(\epsilon^{3}\right)$ SSE formulas do not seem to be consistent with our data. There are several possible explanations for the inconsistencies. One is that the pion masses in our simulations are still too heavy for the SSE formula at this order to be applicable, and the higher-order contributions may not be negligible in this range. The other possibility is that our results still suffer from uncontrolled systematic errors, such as finite-volume effects, especially at the light pion masses. This will be discussed later in Sec. V. We want to point out that our limited number of data points is not sufficient to constrain the chiral fits, which clearly demonstrates the need for calculations at lighter pion masses. Thus we do not regard our results in Table VI as conclusive. Rather, we take it as an indication of the difficulty of chirally extrapolating currently available lattice data.

Also plotted in Fig. 8 are our domain wall results at $m_{\pi}=330 \mathrm{MeV}$ at a coarser lattice spacing [37] $(a \approx$ $0.114 \mathrm{fm}$ ), as well as our updated mixed-action calculations [27] at a lattice spacing of about $0.124 \mathrm{fm}$. These results are roughly consistent with the fine domain wall results, indicating that the discretization errors may be small.

\section{Chiral extrapolations using covariant baryon chiral perturbation theory}

In this section we apply a different formulation of $S U(2)$ chiral effective field theory in the baryon sector, without explicit $\Delta(1232)$ degrees of freedom: covariant $\mathrm{BChPT}$ as introduced in Ref. [63] with a modified version of infrared regularization (IR-scheme). For details about the formalism and differences from the standard infrared regularization introduced by Becher and Leutwyler [34], we refer the reader to Refs. $[32,33,64]$. The expressions for the $m_{\pi}$ dependence of the mean squared isovector Dirac and Pauli radii and the isovector anomalous magnetic moment have been derived in [33] up to order $p^{4}$, i.e. at the next-toleading one-loop accuracy and are collected below. ${ }^{10}$

For the isovector mean squared Dirac radius, the expression is given as

$$
\left(r_{1}^{v}\right)^{2}=B_{c 1}+\left[\left(r_{1}^{v}\right)^{2}\right]^{(3)}+\left[\left(r_{1}^{v}\right)^{2}\right]^{(4)}+\mathcal{O}\left(m_{\pi}^{2}\right),
$$

where

$$
\begin{gathered}
B_{c 1}=-12 d_{6}^{r}(\lambda) \\
{\left[\left(r_{1}^{v}\right)^{2}\right]^{(3)}=-\frac{1}{16 \pi^{2} F_{\pi}^{2} M^{4}}\left[7 g_{A}^{2} M^{4}+2\left(5 g_{A}^{2}+1\right) M^{4} \log \frac{m_{\pi}}{\lambda}+M^{4}-15 g_{A}^{2} m_{\pi}^{2} M^{2}+g_{A}^{2} m_{\pi}^{2}\left(15 m_{\pi}^{2}-44 M^{2}\right) \log \frac{m_{\pi}}{M}\right]} \\
+\frac{g_{A}^{2} m_{\pi}}{16 \pi^{2} F_{\pi}^{2} M^{4} \sqrt{4 M^{2}-m_{\pi}^{2}}}\left[15 m_{\pi}^{4}-74 m_{\pi}^{2} M^{2}+70 M^{4}\right] \arccos \left(\frac{m_{\pi}}{2 M}\right) \\
{\left[\left(r_{1}^{v}\right)^{2}\right]^{(4)}=-\frac{3 c_{6} g_{A}^{2} m_{\pi}^{2}}{16 \pi^{2} F_{\pi}^{2} M_{0}^{4} \sqrt{4 M_{0}^{2}-m_{\pi}^{2}}}\left[m_{\pi}\left(m_{\pi}^{2}-3 M_{0}^{2}\right) \arccos \left(\frac{m_{\pi}}{2 M_{0}}\right)+\sqrt{4 M_{0}^{2}-m_{\pi}^{2}}\left[M_{0}^{2}+\left(M_{0}^{2}-m_{\pi}^{2}\right) \log \frac{m_{\pi}}{M_{0}}\right]\right]}
\end{gathered}
$$

The terms contributing up to and including $\mathcal{O}\left(p^{i}\right)$ are denoted by the superscript $(i)$. Without any loss of generality, the regularization scale $\lambda$ is set equal to $M_{0}$, the nucleon mass in the chiral limit. The low-energy constants $d_{6}$ and $c_{6}$ appear, respectively, in the third- and second-order $\pi N$ effective Lagrangian. The mass function $M$ must be identified with $M_{0}$ if one truncates the previous expression at $\mathcal{O}\left(p^{3}\right)$, whereas at order $p^{4}$, according to Ref. [33], $M$ should be replaced by [32]

\footnotetext{
${ }^{10}$ In Ref. [33] the form factor slopes $\rho_{1}^{v}$ and $\rho_{2}^{v}$ are used, which are related to our notation for $r_{1}^{v}$ and $r_{2}^{v}$ by $\rho_{1}^{v}=\frac{1}{6}\left(r_{1}^{v}\right)^{2}$ and $\rho_{2}^{v}=\frac{1}{6} \kappa_{v} \cdot\left(r_{2}^{v}\right)^{2}$.
} 


$$
\begin{aligned}
M_{N}\left(m_{\pi}\right)= & M_{0}-4 c_{1} m_{\pi}^{2}+\frac{3 g_{A}^{2} m_{\pi}^{3}}{32 \pi^{2} F_{\pi}^{2} \sqrt{4-\frac{m_{\pi}^{2}}{M_{0}^{2}}}}\left(-4+\frac{m_{\pi}^{2}}{M_{0}^{2}}+4 c_{1} \frac{m_{\pi}^{4}}{M_{0}^{3}}\right) \arccos \left(\frac{m_{\pi}}{2 M_{0}}\right) \\
& -\frac{3 m_{\pi}^{4}}{128 \pi^{2} F_{\pi}^{2}}\left[\left(\frac{6 g_{A}^{2}}{M_{0}}-c_{2}\right)+4\left(\frac{g_{A}^{2}}{M_{0}}-8 c_{1}+c_{2}+4 c_{3}\right) \log \left(\frac{m_{\pi}}{\lambda}\right)\right]+4 e_{1}^{r}(\lambda) m_{\pi}^{4}-\frac{3 c_{1} g_{A}^{2} m_{\pi}^{6}}{8 \pi^{2} F_{\pi}^{2} M_{0}^{2}} \log \left(\frac{m_{\pi}}{M_{0}}\right),
\end{aligned}
$$

where $c_{1}, c_{2}$, and $c_{3}$ are second-order low-energy constants and $e_{1}^{r}(\lambda)$ denotes an effective coupling consisting of a combination of fourth order low-energy constants. In our current analysis, we always include terms up to $\mathcal{O}\left(p^{4}\right)$, hence $M$ in all the BChPT expressions presented here should be identified with $M_{N}\left(m_{\pi}\right)$.

The pion-mass dependence of the isovector Pauli radius is given by

$$
\kappa_{v}\left(m_{\pi}\right) \cdot\left(r_{2}^{v}\right)^{2}=\frac{M_{N}}{M_{0}}\left(B_{c 2}+\left[\kappa_{v} \cdot\left(r_{2}^{v}\right)^{2}\right]^{(3)}+\left[\kappa_{v} \cdot\left(r_{2}^{v}\right)^{2}\right]^{(4)}\right)+\mathcal{O}\left(m_{\pi}\right),
$$

where $^{11}$

$$
\begin{gathered}
B_{c 2}=24 M_{0} e_{74}^{r}(\lambda), \\
{\left[\kappa_{v} \cdot\left(r_{2}^{v}\right)^{2}\right]^{(3)}=\frac{g_{A}^{2} M_{0}}{16 \pi^{2} F_{\pi}^{2} M^{5}\left(m_{\pi}^{2}-4 M^{2}\right)}\left[-124 M^{6}+105 m_{\pi}^{2} M^{4}-18 m_{\pi}^{4} M^{2}\right.} \\
\left.+6\left(3 m_{\pi}^{6}-22 M^{2} m_{\pi}^{4}+44 M^{4} m_{\pi}^{2}-16 M^{6}\right) \log \frac{m_{\pi}}{M}\right]+\frac{g_{A}^{2} M_{0}}{8 \pi^{2} F_{\pi}^{2} M^{5} m_{\pi}\left(4 M^{2}-m_{\pi}^{2}\right)^{3 / 2}} \\
\times\left[9 m_{\pi}^{8}-84 M^{2} m_{\pi}^{6}+246 M^{4} m_{\pi}^{4}-216 M^{6} m_{\pi}^{2}+16 M^{8}\right] \arccos \left(\frac{m_{\pi}}{2 M}\right) \\
{\left[\kappa_{v} \cdot\left(r_{2}^{v}\right)^{2}\right]^{(4)}=-\frac{g_{A}^{2} c_{6} m_{\pi}^{3}}{16 \pi^{2} F_{\pi}^{2} M_{0}^{4}\left(4 M_{0}^{2}-m_{\pi}^{2}\right)^{3 / 2}}\left[4 m_{\pi}^{4}-27 m_{\pi}^{2} M_{0}^{2}+42 M_{0}^{4}\right] \arccos \left(\frac{m_{\pi}}{2 M_{0}}\right)+\frac{1}{16 \pi^{2} F_{\pi}^{2} M_{0}^{4}\left(m_{\pi}^{2}-4 M_{0}^{2}\right)}} \\
\times\left[16 c_{4} M_{0}^{7}+52 g_{A}^{2} M_{0}^{6}-4 c_{4} m_{\pi}^{2} M_{0}^{5}-14 c_{6} g_{A}^{2} m_{\pi}^{2} M_{0}^{4}-13 g_{A}^{2} m_{\pi}^{2} M_{0}^{4}+8\left(3 g_{A}^{2}-c_{4} M_{0}\right)\left(m_{\pi}^{2}-4 M_{0}^{2}\right) M_{0}^{4}\right. \\
\left.\quad \times \log \frac{m_{\pi}}{M_{0}}+4 c_{6} g_{A}^{2} m_{\pi}^{4} M_{0}^{2}-g_{A}^{2}\left(m_{\pi}^{2}-4 M_{0}^{2}\right)\left(4 c_{6} m_{\pi}^{4}-3 c_{6} m_{\pi}^{2} M_{0}^{2}+24 M_{0}^{4}\right) \log \frac{m_{\pi}}{M_{0}}\right]
\end{gathered}
$$

For the isovector anomalous magnetic moment, the $\mathcal{O}\left(p^{4}\right)$ BChPT expression is

$$
\kappa_{v}=\frac{M_{N}}{M_{0}}\left[c_{6}-16 M_{0} m_{\pi}^{2} e_{106}^{r}(\lambda)+\delta \kappa_{v}^{(3)}+\delta \kappa_{v}^{(4)}\right]+\mathcal{O}\left(m_{\pi}^{3}\right)
$$

where

$$
\begin{aligned}
\delta \kappa_{v}^{(3)} & =\frac{g_{A}^{2} m_{\pi}^{2} M_{0}}{8 \pi^{2} F_{\pi}^{2} M^{3}}\left[\left(3 m_{\pi}^{2}-7 M^{2}\right) \log \frac{m_{\pi}}{M}-3 M^{2}\right]-\frac{g_{A}^{2} m_{\pi} M_{0}}{8 \pi^{2} F_{\pi}^{2} M^{3} \sqrt{4 M^{2}-m_{\pi}^{2}}}\left[3 m_{\pi}^{4}-13 M^{2} m_{\pi}^{2}+8 M^{4}\right] \arccos \left(\frac{m_{\pi}}{2 M}\right), \\
\delta \kappa_{v}^{(4)}= & -\frac{m_{\pi}^{2}}{32 \pi^{2} F_{\pi}^{2} M_{0}^{2}}\left[4 g_{A}^{2}\left(c_{6}+1\right) M_{0}^{2}-g_{A}^{2}\left(5 c_{6} m_{\pi}^{2}+28 M_{0}^{2}\right) \log \frac{m_{\pi}}{M_{0}}+4 M_{0}^{2}\left(2 c_{6} g_{A}^{2}+7 g_{A}^{2}+c_{6}-4 c_{4} M_{0}\right) \log \frac{m_{\pi}}{\lambda}\right] \\
& -\frac{g_{A}^{2} c_{6} m_{\pi}^{3}}{32 \pi^{2} F_{\pi}^{2} M_{0}^{2} \sqrt{4 M_{0}^{2}-m_{\pi}^{2}}}\left(5 m_{\pi}^{2}-16 M_{0}^{2}\right) \arccos \left(\frac{m_{\pi}}{2 M_{0}}\right) .
\end{aligned}
$$

\footnotetext{
${ }^{11}$ We note that $\mathcal{C}$ in Eq. (55) is equivalent to $e_{74}^{r}(\lambda)$.
} 
TABLE VII. Input values for the covariant baryon chiral fits.

\begin{tabular}{ccccc}
\hline \hline$g_{A}$ & $F_{\pi}[\mathrm{GeV}]$ & $c_{2}\left[\mathrm{GeV}^{-1}\right]$ & $c_{3}\left[\mathrm{GeV}^{-1}\right]$ & $c_{4}\left[\mathrm{GeV}^{-1}\right]$ \\
\hline 1.2 & 0.0862 & 3.2 & -3.4 & 3.5 \\
\hline \hline
\end{tabular}

TABLE VIII. Low-energy constants determined from the fit to the pion-mass dependence of the nucleon mass using the $\mathcal{O}\left(p^{4}\right)$ BChPT expression. Only the domain wall results on the fine lattices are included in the "Lattice only" fit, and in the "Lattice + Exp" fit we impose that the curve goes through the physical point.

\begin{tabular}{l|ccc}
\hline \hline Fit & $\begin{array}{c}M_{0} \\
{[\mathrm{GeV}]}\end{array}$ & $\begin{array}{c}c_{1} \\
{\left[\mathrm{GeV}^{-1}\right]}\end{array}$ & $\begin{array}{c}e_{1}^{r}(\lambda=1 \mathrm{GeV}) \\
{\left[\mathrm{GeV}^{-3}\right]}\end{array}$ \\
\hline Lattice only & $0.883(79)$ & $-1.01(26)$ & $1.1(1.3)$ \\
Lattice + Exp & $0.8726(29)$ & $-1.049(40)$ & $0.90(32)$ \\
\hline \hline
\end{tabular}

Note that $\frac{M_{N}}{M_{0}} c_{6}$ is equivalent to $\kappa_{v}^{0}$ in Eq. (40), where $M_{N}$ is the physical nucleon mass and $M_{0}$ is the nucleon mass in the chiral limit.

In our chiral extrapolations, we treat $g_{A}, F_{\pi}, c_{2}, c_{3}$, and $c_{4}$ as input parameters. The available information about the chiral limit values of $g_{A}$ and $F_{\pi}$ have been discussed in the previous section. We set the second-order couplings consistently with Refs. [65-67]. ${ }^{12}$ We summarize these values in Table VII.

We determine $M_{0}, c_{1}$, and $e_{1}^{r}(\lambda)$ appearing in $M_{N}\left(m_{\pi}\right)$ by fitting the nucleon masses from the three fine DWF ensembles to Eq. (60). The fit values are tabulated in Table VIII and the resulting fit curve is shown in Fig. 9. The fit (denoted as "Lattice only" in the table) is in excellent agreement with the physical nucleon mass, but the small number of data points included in the fit gives substantial statistical errors. To better constrain the value of $M_{0}$, which is needed in the subsequent fits, we also fit the data with the experimental point as a constraint (denoted as "Lattice + Exp"). The results are again shown in Table VIII. The two fits give consistent results, and we will use central values of $M_{0}, c_{1}$ and $e_{1}^{r}(\lambda)$ determined from the "Lattice + Exp" fit subsequently.

For comparison, we also plot the coarse $(a=0.114 \mathrm{fm})$ domain wall result at $m_{\pi} \approx 330 \mathrm{MeV}$, as well as the mixed-action results [54] at $a=0.124 \mathrm{fm}$ in Fig. 9. We see that these results are qualitatively very consistent, indicating the discretization errors are small.

We determine the remaining four low-energy constants, $c_{6}, d_{6}^{r}(\lambda), e_{74}^{r}(\lambda)$, and $e_{106}^{r}(\lambda)$, from a simultaneous fit to $\left(r_{1}^{v}\right)^{2}, \kappa_{v} \cdot\left(r_{2}^{v}\right)^{2}$, and $\kappa_{v}$ using $\mathcal{O}\left(p^{4}\right)$ BChPT expressions presented previously, with the results shown in Table IX. The large $\chi^{2} /$ dof value indicates that the $\mathcal{O}\left(p^{4}\right)$ BChPT does not describe our data either. We compare the chiral

\footnotetext{
${ }^{12}$ For a discussion about the value of $c_{3}$, see $[68,69]$.
}

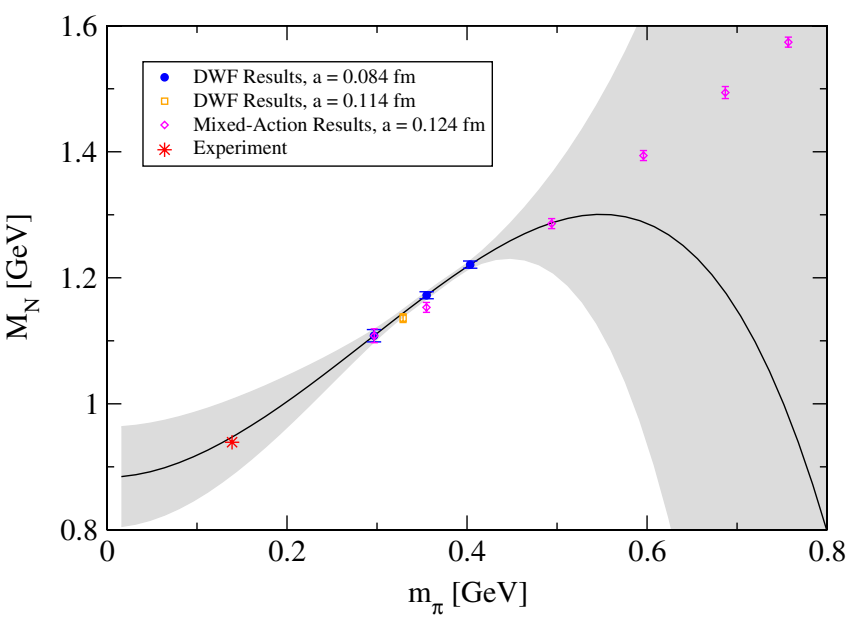

FIG. 9 (color online). Chiral extrapolation for the nucleon mass using the $\mathcal{O}\left(p^{4}\right)$ BChPT formula in Eq. (60). The solid line is the fit to only the fine domain wall data (solid circles). The square is the coarse domain wall result, and the diamonds are the mixed-action results from Ref. [54].

TABLE IX. Fit parameters for the simultaneous fit to $\left(r_{1}^{v}\right)^{2}$, $\kappa_{v} \cdot\left(r_{2}^{v}\right)^{2}$, and $\kappa_{v}$ using the $\mathcal{O}\left(p^{4}\right)$ covariant baryon formula. We have set $\lambda=M_{0}$.

\begin{tabular}{ccccc}
\hline \hline$\chi^{2} /$ dof & $c_{6}$ & $\begin{array}{c}d_{6}^{r}(\lambda) \\
{\left[\mathrm{GeV}^{-2}\right]}\end{array}$ & $\begin{array}{c}e_{74}^{r}(\lambda) \\
{\left[\mathrm{GeV}^{-3}\right]}\end{array}$ & $\begin{array}{c}e_{106}^{r}(\lambda) \\
{\left[\mathrm{GeV}^{-3}\right]}\end{array}$ \\
\hline $7.3(2.4)$ & $4.290(46)$ & $0.839(7)$ & $1.350(45)$ & $-0.132(37)$ \\
\hline \hline
\end{tabular}

extrapolations using both the BChPT formula and the $\mathcal{O}\left(\epsilon^{3}\right)$ SSE formula in Fig. 10. The solid curves with error bands are the results of the BChPT simultaneous fit, and the dashed curves are the SSE fits using Eqs. (42), (43), and (40) as described in Sec. III C 1. It appears that both the SSE and BChPT expressions are not compatible with our data, but since many of the low-energy constants in BChPT are fixed from phenomenology or the nucleon mass, the fit is better constrained than that using the $\mathcal{O}\left(\epsilon^{3}\right)$ SSE expressions. This is especially important for $\kappa_{v}$, for which the SSE expression involves more parameters than currently available lattice data. Nevertheless, both formulations fail to describe our data at this mass range.

\section{ISOSCALAR FORM FACTORS}

Since we have not calculated the disconnected contributions to the three-point functions for the form factors, in this section we give results for the isoscalar form factors as defined in Eq. (5) from the connected diagrams only. The renormalized results (using the renormalization factors discussed in Sec. III A) in terms of the quark flavor content $F_{1,2}^{u+d}\left(Q^{2}\right)=F_{1,2}^{u}\left(Q^{2}\right)+F_{1,2}^{d}\left(Q^{2}\right) \equiv 3 F_{1,2}^{s}\left(Q^{2}\right)$ are presented in Tables XIII, XIV, and XV. First, we study the $Q^{2}$ dependence of both the isoscalar Dirac and Pauli form 


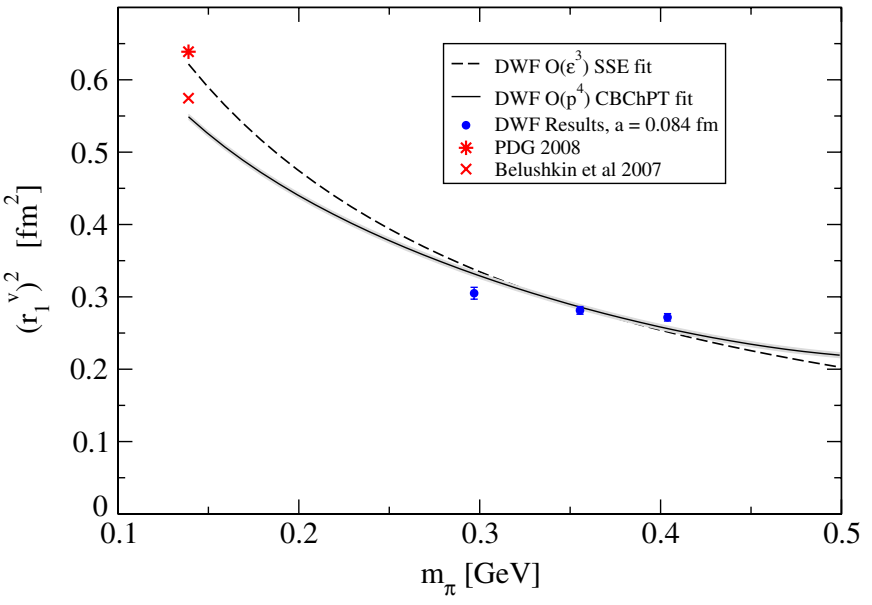

(a)

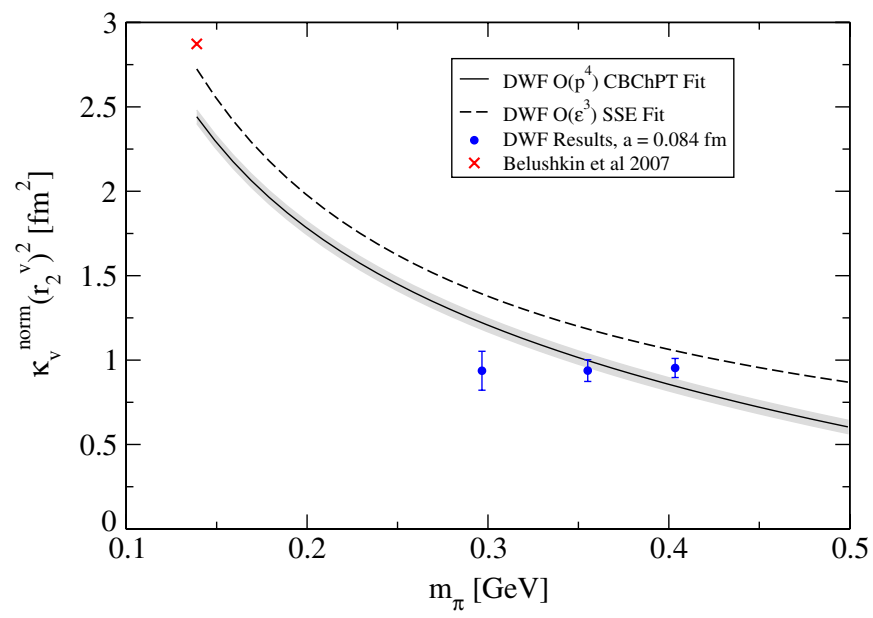

(b)

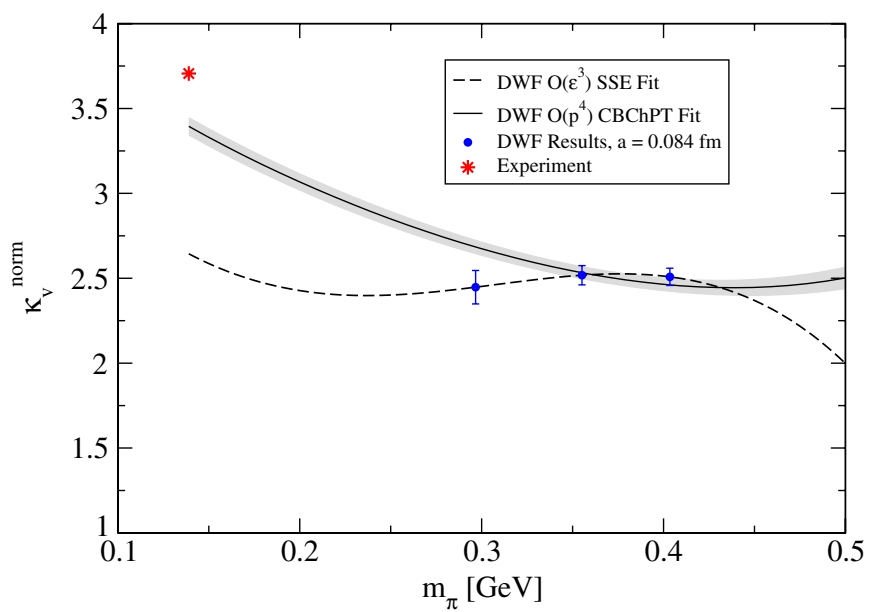

(c)

FIG. 10 (color online). Simultaneous fit to the isovector Dirac radius $\left(r_{1}^{v}\right)^{2}$ (a), Pauli radius $\kappa_{v} \cdot\left(r_{2}^{v}\right)^{2}$ (b), and the anomalous magnetic moment $\kappa_{v}$ (c) using the covariant baryon formula (solid lines). The dashed lines show the SSE formula fits without the constant term for $\kappa_{v} \cdot\left(r_{2}^{v}\right)^{2}$.

factors using phenomenological models, and then discuss briefly the chiral extrapolations of the results.

\section{A. $Q^{2}$ dependence}

Unlike the isovector Dirac form factor, $F_{1}^{u+d}(0)$ is not set to the known value of 3 . Thus we perform dipole fits to $F_{1}^{u+d}\left(Q^{2}\right)$ separately to each ensemble using the formula in Eq. (30). Similar to the isovector case (see Sec. III B), the dipole Ansatz describes the data reasonably well at small $Q^{2}$ values, typically below $0.6 \mathrm{GeV}^{2}$. As large $Q^{2}$ values are included in the fit, the fit quality becomes worse, but the fit parameters do not change significantly. Furthermore, the fitted values of $F_{1}^{u+d}(0)$ are very consistent with the expected value of 3 .

To demonstrate the quality of the fits, in Fig. 11 we show the dipole fits to all the $Q^{2}$ values. One can see that the data are reasonably well described by the fit curves. Also plotted is the phenomenological fit to experimental data using the parameterization in Ref. [51], although we note that no error estimate is provided and the empirical analysis involves many potential systematic errors discussed in the introduction. To determine the isoscalar mean squared Dirac radii, we follow the same reasoning as in Sec. III B and obtain them from the dipole fits with a cut at $Q^{2} \leq$ $0.5 \mathrm{GeV}^{2}$. The results are shown in Table X.

In experiments, the isoscalar Pauli form factor shows a notable bump at $Q^{2} \approx 0.4 \mathrm{GeV}^{2}$ (solid curve in Fig. 12), although again there are no error estimates. Our data are too noisy to distinguish this feature at this moment. In fact, the results, shown in Fig. 12, are rather flat. We show the constant fits to each ensemble separately, and find that the constants are consistent with zero within 2 standard deviations. The error band corresponds to the constant fit to the $m_{\pi}=297 \mathrm{MeV}$ data.

If we restrict the fits to only the small $Q^{2}$ region $\left(\leq 0.5 \mathrm{GeV}^{2}\right.$ ), we are able to perform linear fits to the data and obtain both $\kappa_{s} \cdot\left(r_{2}^{s}\right)^{2}$ (from the slope) and $\kappa_{s}$ 


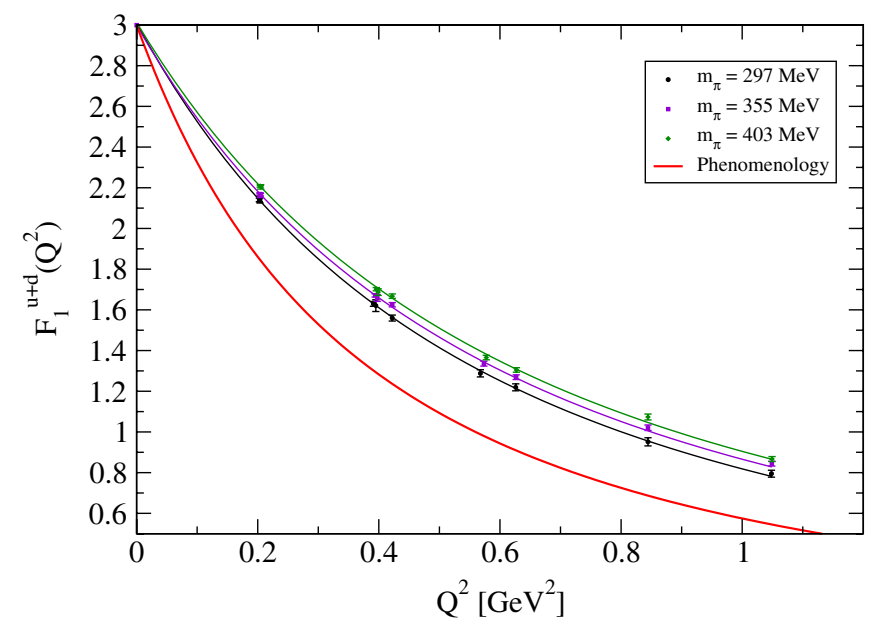

FIG. 11 (color online). The isoscalar Dirac form factor, $F_{1}^{u+d}\left(Q^{2}\right)$, with dipole fits. The thick solid (red) curve is a phenomenological fit to experimental data [51].

(from the intercept), the results of which are also shown in Table X. ${ }^{13}$

\section{B. Chiral extrapolations}

\section{Chiral extrapolations using $\mathcal{O}\left(\epsilon^{3}\right)$ small scale expansion}

As is well known in ChPT (e.g. see the discussion in [44]), chiral dynamics in the isoscalar form factors of the nucleon starts at the 3-pion cut, i.e. at two-loop level, corresponding to $\mathcal{O}\left(\epsilon^{5}\right)$ in the power-counting of SSE. Hence, although the $\mathcal{O}\left(\epsilon^{3}\right)$ SSE expressions for the pionmass and momentum transfer dependence of the isoscalar Dirac and Pauli form factors have also been derived in [44] and given as

$$
F_{1}^{s}\left(Q^{2}\right)=1+\tilde{B}_{1} \frac{Q^{2}}{\left(4 \pi F_{\pi}\right)^{2}}, \quad F_{2}^{s}\left(Q^{2}\right)=\kappa_{s},
$$

they cannot be utilized for chiral extrapolations. Therefore, in this section, we simply extrapolate linearly in $m_{\pi}^{2}$ the mean squared Dirac radius to the physical point. This is shown in Fig. 13(a), where we can see that the linear extrapolation gives a result at the physical pion mass which is much lower than the empirical value. Similarly, we perform a linear extrapolation for $\kappa_{s} \cdot\left(r_{2}^{s}\right)^{2}$, which is shown in Fig. 13(b).

For $\kappa_{s}$ beyond order $\epsilon^{3}$, additional terms arise including a term linear in the quark mass. Following Ref. [52], we write

$$
\kappa_{s}=\kappa_{s}^{0}-8 E_{2} M_{N} m_{\pi}^{2},
$$

where $\kappa_{s}^{0}$ and $E_{2}$ are two unknown LECs. This linear

\footnotetext{
${ }^{13}$ Like in the isovector case, the anomalous magnetic moment quoted here is normalized to the physical nuclear magneton according to Eq. (41).
}

TABLE X. Results for the isoscalar Dirac and Pauli mean squared radii and the anomalous magnetic moment. A dipole fit with a $Q^{2}$ cutoff at $0.5 \mathrm{GeV}^{2}$ is used to determine $\left(r_{1}^{s}\right)^{2}$. Linear fits to $F_{2}^{s}$ with $Q^{2} \leq 0.5 \mathrm{GeV}^{2}$ are used to determine $\kappa_{s} \cdot\left(r_{2}^{s}\right)^{2}$ and $\kappa_{s}$. The results shown below have been normalized to the physical nuclear magneton.

\begin{tabular}{l|cc|ccr}
\hline \hline$m_{\pi}$ & & $\left(r_{1}^{s}\right)^{2}$ & \multicolumn{3}{|c}{$\kappa_{s}^{\text {norm }} \cdot\left(r_{2}^{s}\right)^{2}$} \\
{$[\mathrm{MeV}]$} & $\chi^{2} /$ dof & {$\left[\mathrm{fm}^{2}\right]$} & $\chi^{2} /$ dof & {$\left[\mathrm{fm}^{2}\right]$} & $\kappa_{s}^{\text {norm }}$ \\
\hline 297 & $0.12(35)$ & $0.428(5)$ & $3.3(2.1)$ & $-0.021(21)$ & $-0.038(37)$ \\
355 & $0.97(98)$ & $0.403(3)$ & $1.4(1.4)$ & $-0.015(11)$ & $-0.030(22)$ \\
403 & $1.7(1.3)$ & $0.385(3)$ & $2.2(1.7)$ & $-0.003(11)$ & $0.011(21)$ \\
\hline \hline
\end{tabular}

dependence describes our data well, as is shown in Fig. 13(c).

\section{Chiral extrapolations in covariant baryon chiral perturbation theory}

The BChPT formulas up to $\mathcal{O}\left(p^{4}\right)$ for $\left(r_{1}^{s}\right)^{2},\left(r_{2}^{s}\right)^{2}$, and $\kappa_{s}$ have also been derived in $[33,64]$. We collect them here for completeness. We note, however, that the next-to-leading one-loop BChPT results for the isoscalar form factors of the nucleon as presented in this section-just as in the case of the leading one-loop SSE-analysis discussed in the previous section-do not contain their dominant chiral dynamics arising from the 3-pion cut. Such effects would only become visible at the two-loop level, i.e. starting at $\mathcal{O}\left(p^{5}\right)$ in BChPT. The results presented here are therefore to be interpreted with care, as several important contributions with potentially large impact on the chiral extrapolation functions are not included at this order. For the isoscalar mean squared Dirac radius, the BChPT expression is given by

$$
\left(r_{1}^{s}\right)^{2}=B_{c 1}^{s}+\left[\left(r_{1}^{s}\right)^{2}\right]^{(3)}+\left[\left(r_{1}^{s}\right)^{2}\right]^{(4)},
$$

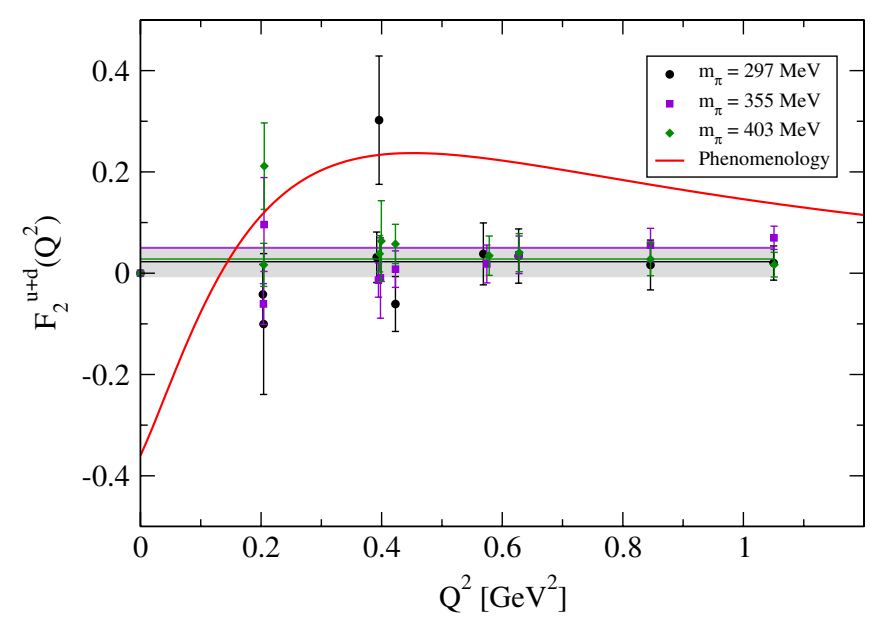

FIG. 12 (color online). The isoscalar Pauli form factor, $F_{2}^{u+d}\left(Q^{2}\right)$, with constant fits. Only the error band for the fit to the $m_{\pi}=297 \mathrm{MeV}$ ensemble is shown. The thick solid (red) curve is a phenomenological fit to experimental data [51]. 


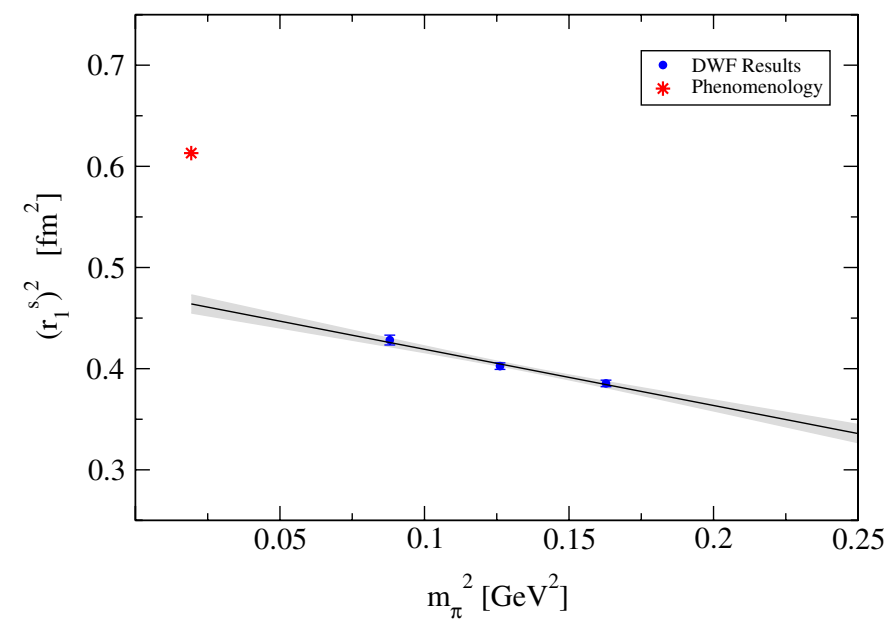

(a)

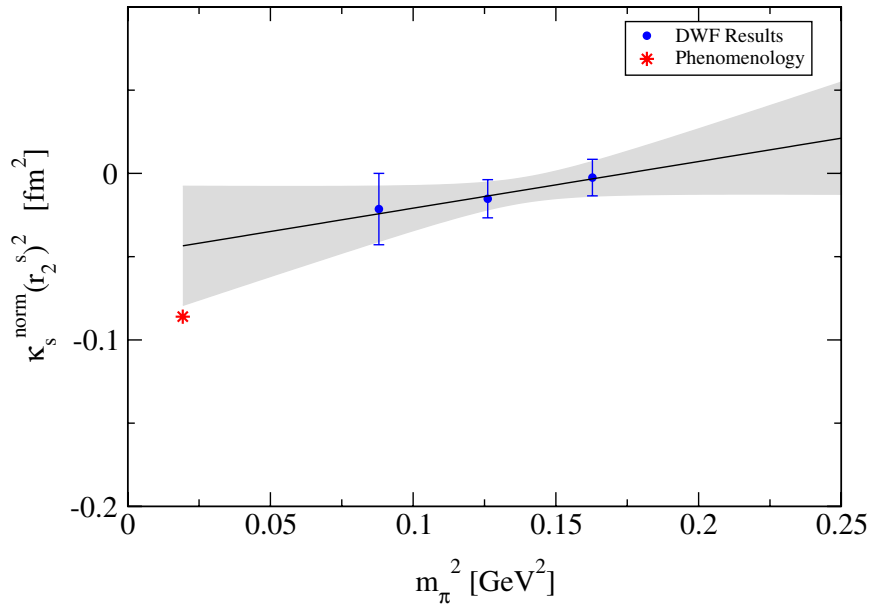

(b)

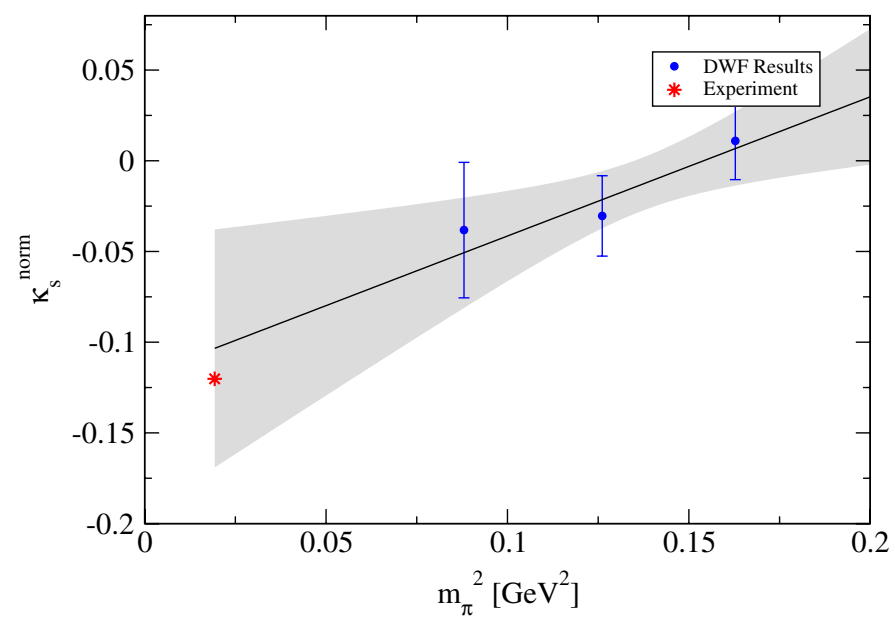

(c)

FIG. 13 (color online). The linear extrapolations for the isoscalar Dirac radius $\left(r_{1}^{s}\right)^{2}$ (a), Pauli radius $\kappa_{s}\left(r_{2}^{s}\right)^{2}$ (b), and the anomalous magnetic moment $\kappa_{s}$ (c). In (a), (b) the stars indicate the phenomenological values obtained in Ref. [6]. In (c) the star indicates the experimental value [56].

where

$$
\begin{aligned}
& B_{c 1}^{s}=-24 d_{7}, \\
& {\left[\left(r_{1}^{s}\right)^{2}\right]^{(3)=} } \frac{3 g_{A}^{2} m_{\pi}^{2}}{16 \pi^{2} F_{\pi}^{2} M^{4}\left(m_{\pi}^{2}-4 M^{2}\right)}\left[5 m_{\pi}^{2} M^{2}-18 M^{4}+\frac{m_{\pi}\left(5 m_{\pi}^{4}-34 M^{2} m_{\pi}^{2}+54 M^{4}\right)}{\sqrt{4 M^{2}-m_{\pi}^{2}}} \arccos \left(\frac{m_{\pi}}{2 M}\right)\right. \\
&\left.-\left(m_{\pi}^{2}-4 M^{2}\right)\left(5 m_{\pi}^{2}-4 M^{2}\right) \log \frac{m_{\pi}}{M}\right], \\
& {\left[\left(r_{1}^{s}\right)^{2}\right]^{(4)}=} \frac{9 g_{A}^{2} \kappa_{s}^{0} m_{\pi}^{2}}{16 \pi^{2} F_{\pi}^{2} M_{0}^{4}}\left[M_{0}^{2}+\left(M_{0}^{2}-m_{\pi}^{2}\right) \log \frac{m_{\pi}}{M_{0}}+\frac{m_{\pi}\left(m_{\pi}^{2}-3 M_{0}^{2}\right)}{\sqrt{4 M_{0}^{2}-m_{\pi}^{2}}} \arccos \left(\frac{m_{\pi}}{2 M_{0}}\right)\right] .
\end{aligned}
$$

Here, again, when the expression is truncated at $\mathcal{O}\left(p^{3}\right), M$ should be identified with $M_{0}$, while at $\mathcal{O}\left(p^{4}\right)$, it should be replaced by $M_{N}\left(m_{\pi}\right)$ in Eq. (60). Similarly, for $\kappa_{s} \cdot\left(r_{2}^{s}\right)^{2}$, we have 


$$
\kappa_{s} \cdot\left(r_{2}^{s}\right)^{2}=\frac{M_{N}}{M_{0}}\left(B_{c 2}^{s}+\left[\kappa_{s} \cdot\left(r_{2}^{s}\right)^{2}\right]^{(3)}+\left[\kappa_{s} \cdot\left(r_{2}^{s}\right)^{2}\right]^{(4)}\right),
$$

with

$$
\begin{aligned}
B_{c 2}^{s} & =48 M_{0} e_{54}, \\
{\left[\kappa_{s} \cdot\left(r_{2}^{s}\right)^{2}\right]^{(3)}=} & \frac{3 g_{A}^{2} m_{\pi}^{2} M_{0}}{16 \pi^{2} F_{\pi}^{2} M^{5}\left(4 M^{2}-m_{\pi}^{2}\right)}\left[\frac{m_{\pi}\left(6 m_{\pi}^{4}-40 M^{2} m_{\pi}^{2}+60 M^{4}\right)}{\sqrt{4 M^{2}-m_{\pi}^{2}}} \arccos \left(\frac{m_{\pi}}{2 M}\right)\right. \\
& \left.-2\left(10 M^{4}-3 m_{\pi}^{2} M^{2}+\left(4 M^{2}-m_{\pi}^{2}\right)\left(2 M^{2}-3 m_{\pi}^{2}\right) \log \frac{m_{\pi}}{M}\right)\right], \\
{\left[\kappa_{s} \cdot\left(r_{2}^{s}\right)^{2}\right]^{(4)}=} & \frac{3 \kappa_{s}^{0} g_{A}^{2} m_{\pi}^{2}}{16 \pi^{2} F_{\pi}^{2} M_{0}^{4}\left(m_{\pi}^{2}-4 M_{0}^{2}\right)}\left[-\frac{m_{\pi}\left(4 m_{\pi}^{4}-27 M_{0}^{2} m_{\pi}^{2}+42 M_{0}^{4}\right)}{\sqrt{4 M_{0}^{2}-m_{\pi}^{2}}} \arccos \left(\frac{m_{\pi}}{2 M_{0}}\right)\right. \\
+ & \left.14 M_{0}^{4}-4 m_{\pi}^{2} M_{0}^{2}+\left(m_{\pi}^{2}-4 M_{0}^{2}\right)\left(4 m_{\pi}^{2}-3 M_{0}^{2}\right) \log \frac{m_{\pi}}{M_{0}}\right] .
\end{aligned}
$$

The BChPT expression for the isoscalar anomalous magnetic moment is written as

$$
\kappa_{s}=\frac{M_{N}}{M_{0}}\left[\kappa_{s}^{0}-16 M_{0} m_{\pi}^{2} e_{105}^{r}(\lambda)+\delta \kappa_{s}^{(3)}+\delta \kappa_{s}^{(4)}\right],
$$

where

$$
\begin{gathered}
\delta \kappa_{s}^{(3)}=-\frac{3 g_{A}^{2} m_{\pi}^{2} M_{0}}{8 \pi^{2} F_{\pi}^{2} M^{3}}\left[\frac{m_{\pi}\left(m_{\pi}^{2}-3 M^{2}\right)}{\sqrt{4 M^{2}-m_{\pi}^{2}}} \arccos \left(\frac{m_{\pi}}{2 M}\right)+M^{2}+\left(M^{2}-m_{\pi}^{2}\right) \log \frac{m_{\pi}}{M}\right], \\
\delta \kappa_{s}^{(4)}=\frac{3 g_{A}^{2} m_{\pi}^{2}}{32 \pi^{2} F_{\pi}^{2} M_{0}^{2}}\left[4 M_{0}^{2}+\kappa_{s}^{0}\left(3 m_{\pi}^{2}-4 M_{0}^{2}\right) \log \frac{m_{\pi}}{M_{0}}-\kappa_{s}^{0} \frac{m_{\pi}\left(3 m_{\pi}^{2}-8 M_{0}^{2}\right)}{\sqrt{4 M_{0}^{2}-m_{\pi}^{2}}} \arccos \left(\frac{m_{\pi}}{2 M_{0}}\right)\right] .
\end{gathered}
$$

As in the isovector case, we use the values in Table VII as input in the extrapolations, leaving $\kappa_{s}^{0}, d_{7}, e_{54}$ and $e_{105}^{r}(\lambda)$ as free parameters. Since $\left(r_{1}^{s}\right)^{2}, \kappa_{s} \cdot\left(r_{2}^{s}\right)^{2}$, and $\kappa_{s}$ all contain the low-energy constant $\kappa_{s}^{0}$, naively we should perform a simultaneous fit to all three quantities, as we have done for the isovector case. However, as stated earlier, the dominant chiral dynamics for the isoscalar quantities only appears at $\mathcal{O}\left(p^{5}\right)$. We do not expect these $\mathcal{O}\left(p^{3}\right)$ expressions to describe our data. In fact, the simultaneous fit to these three quantities gives a $\chi^{2} /$ dof of about 9 (see Table XI), showing the difficulty in fitting these quantities consistently. Looking closely at each quantity separately,

TABLE XI. Fit parameters from the simultaneous fit to $\left(r_{1}^{s}\right)^{2}$, $\kappa_{s} \cdot\left(r_{2}^{s}\right)^{2}$ and $\kappa_{s}$ using Eqs. (69), (72), and (76).

\begin{tabular}{lcccc}
\hline \hline$\chi^{2} /$ dof & $\kappa_{s}^{0}$ & $\begin{array}{c}d_{7} \\
{\left[\mathrm{GeV}^{-2}\right]}\end{array}$ & $\begin{array}{c}e_{54} \\
{\left[\mathrm{GeV}^{-3}\right]}\end{array}$ & $\begin{array}{c}e_{105}^{r}\left(\lambda=M_{0}\right) \\
{\left[\mathrm{GeV}^{-3}\right]}\end{array}$ \\
\hline $8.5(2.6)$ & $-0.172(23)$ & $-0.458(24)$ & $-0.0159(41)$ & $0.598(26)$ \\
\hline \hline
\end{tabular}

we find that independent fits to $\left(r_{1}^{s}\right)^{2}, \kappa_{s} \cdot\left(r_{2}^{s}\right)^{2}$ and $\kappa_{s}$ lead to an inconsistency in the estimation of the common parameter $\kappa_{s}^{0}$, as shown in Table XII. For demonstrative purposes, we compare the resulting fit curves from the simultaneous fit and the independent fits in Fig. 14, from which we see that the independent fits provide reasonable extrapolations for the data, while the simultaneous fit misses the data points badly, indicating inconsistencies of the BChPT expressions at this order. We also note that the

TABLE XII. Fit parameters from independent fits to $\left(r_{1}^{s}\right)^{2}, \kappa_{s}$. $\left(r_{2}^{s}\right)^{2}$ and $\kappa_{s}$ using Eqs. (69), (72), and (76).

\begin{tabular}{lccc}
\hline \hline & $\chi^{2} /$ dof & $\kappa_{s}^{0}$ & $d_{7}\left[\mathrm{GeV}^{-2}\right]$ \\
$\left(r_{1}^{s}\right)^{2}$ & $0.2(9)$ & $2.67(44)$ & $-0.581(19)$ \\
& $\chi^{2} /$ dof & $\kappa_{s}^{0}$ & $e_{54}\left[\mathrm{GeV}^{-3}\right]$ \\
$\kappa_{s} \cdot\left(r_{2}^{s}\right)^{2}$ & $0.08(55)$ & $1.6(2.0)$ & $-0.055(44)$ \\
& $\chi^{2} /$ dof & $\kappa_{s}^{0}$ & $e_{105}^{r}\left(\lambda=M_{0}\right)\left[\mathrm{GeV}^{-3}\right]$ \\
$\kappa_{s}$ & $0.4(1.3)$ & $-0.247(53)$ & $0.506(63)$ \\
\hline \hline
\end{tabular}




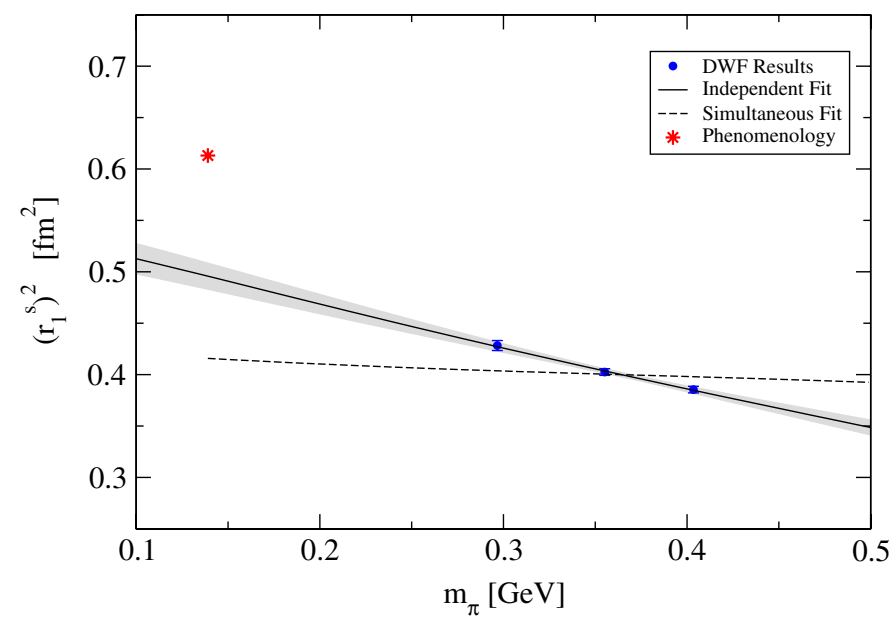

(a)

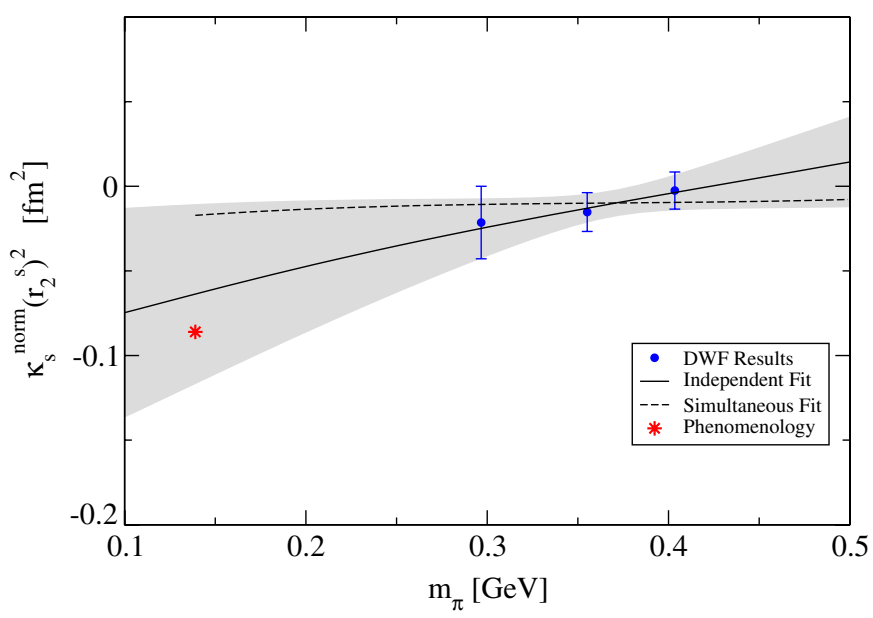

(b)

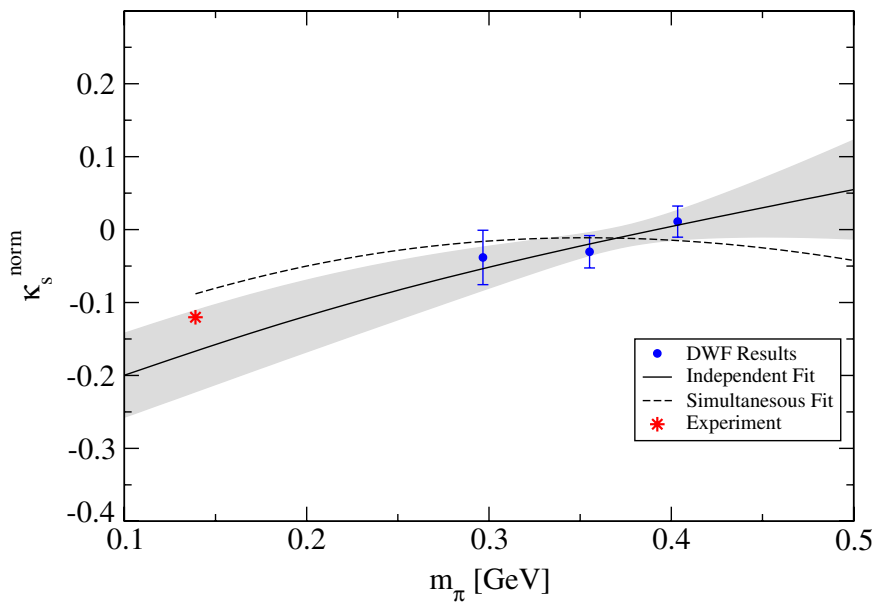

(c)

FIG. 14 (color online). $\quad \mathcal{O}\left(p^{4}\right)$ BChPT fits to the isoscalar Dirac radius $\left(r_{1}^{s}\right)^{2}$ (a), Pauli radius $\kappa_{s}\left(r_{2}^{s}\right)^{2}(\mathrm{~b})$, and the anomalous magnetic moment $\kappa_{s}$ (c). The solid lines are fits to the three quantities separately with the resulting fit parameters summarized in Table XII. The dashed lines are simultaneous fits with the parameters summarized in Table XI.

extrapolated value for $\left(r_{1}^{s}\right)^{2}$ at the physical pion mass is about $20 \%$ lower than the phenomenological value. These observations lead us to conclude that the BChPT expressions at $\mathcal{O}\left(p^{3}\right)$ are not applicable in the pion-mass range of our calculation. Of course, since we have not included the disconnected diagrams in our calculations, there are uncontrolled systematic errors which may also affect the pion-mass dependence. Further investigations are required to draw definitive conclusions for these isoscalar quantities.

\section{SYSTEMATIC ERRORS}

\section{A. Effect of the excited states}

The correlation functions may have systematic bias due to the excited and/or unphysical oscillating states $[24,28,70]$. To control it, we solve the overdetermined system separately for each location of the operator and examine the plateau for the form factors. Examples are shown in Fig. 15. Because of the tuning of the quark sources, the contaminations from states other than the ground state are suppressed and do not contribute to the matrix element plateaus close to their centers.

To put quantitative bounds on possible bias, we study the excited states in the nucleon correlators. The nucleon twopoint correlation functions have very precise information on the presence of the nonground state contamination. For example, with our current statistics the parameters of a fit with three states are well constrained:

$$
\begin{aligned}
C_{2 \mathrm{pt}}(t ; P)= & Z_{0}(P) e^{-E_{0} t}+Z_{1}(P) e^{-E_{1} t} \\
& +(-1)^{t} Z_{\mathrm{osc}}(P) e^{-E_{\mathrm{osc}} t}, \quad Z_{0,1}>0,
\end{aligned}
$$

where $Z_{0}, Z_{1}$, and $Z_{\text {osc }}$ denote the overlap of the nucleon 


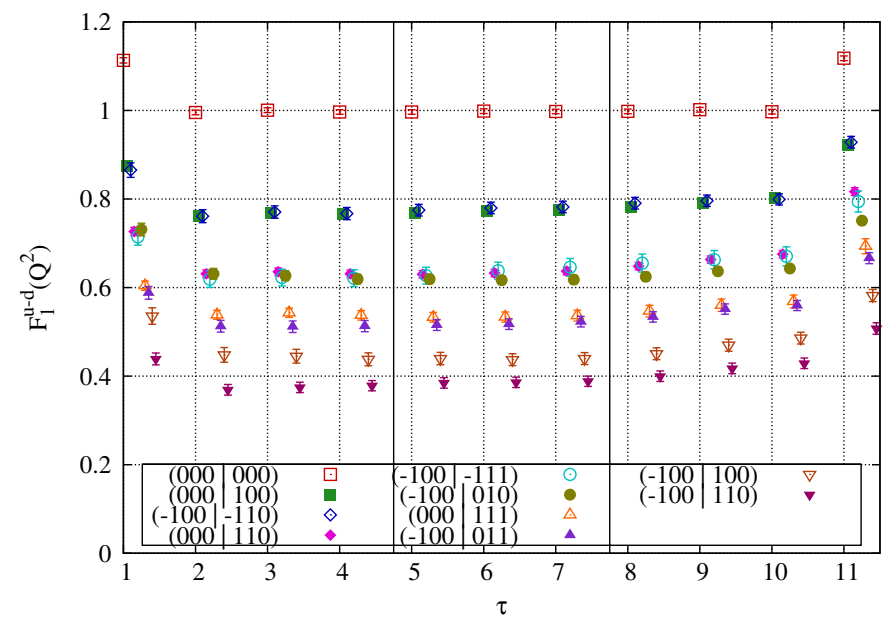

(a)

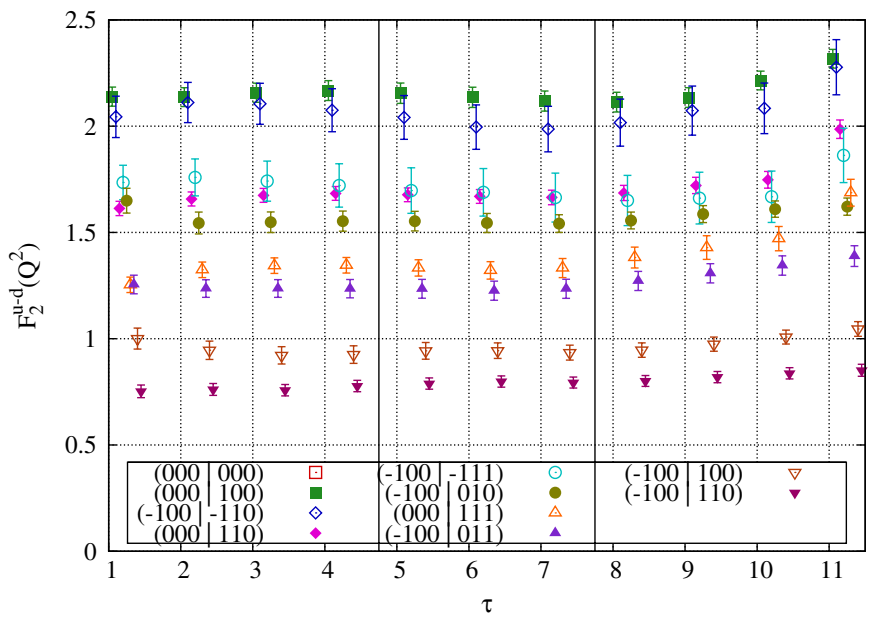

(b)

FIG. 15 (color online). Nucleon isovector Dirac (a) and Pauli (b) form factor plateaus for the lightest $m_{\pi}=297 \mathrm{MeV}$ ensemble.

interpolating field with the ground, the first excited and the unphysical oscillating states, respectively. Having estimated the energy gap $\Delta E_{10}(P)=E_{1}(P)-E_{0}(P)$ and the magnitude of the contamination $Z_{1}(P) / Z_{0}(P)$, one can put bounds on the excited state contribution to the matrix elements computed from the two- and three-point lattice nucleon correlators.

The ratio formula (23) for physical matrix elements has two factors: $R^{V^{\mu}} \equiv R_{N} R_{A}$. Excited states can potentially contribute to either one. First, we study the asymmetry ratio, $R_{A}$, defined in Eq. (22). As was pointed out above, this factor compensates the asymmetric $\tau$ dependence in $R_{N}$, and in the absence of excited states it would be equal to $\exp \left[-\left(E^{\prime}-E\right)(\tau-T / 2)\right]$. Although this factor involves different two-point functions, their excited state contribu- tions appear to cancel each other to a large extent, as shown in Fig. 16. Figure 16(a) shows the ratio of $R_{A}$ to the exponential result in the absence of excited states

$$
\frac{R_{A}(\tau)}{e^{-\left(E^{\prime}-E\right)(\tau-T / 2)}}=\frac{\sqrt{\frac{C_{2 \mathrm{pt}}(T-\tau, P) C_{2 \mathrm{pt}}\left(\tau, P^{\prime}\right)}{C_{2 \mathrm{pt}}\left(T-\tau, P^{\prime}\right) C_{2 \mathrm{pt}}(\tau, P)}}}{e^{-\left(E^{\prime}-E\right)(\tau-T / 2)}}
$$

where $\left(E^{\prime}-E\right)$ in the denominator is determined by the best fit to $R_{A}$ in the range $3 \leq \tau \leq 6$. The fact that this ratio is unity to within $1 \%$ over a plateau from $3 \leq \tau \leq 9$ indicates that excited state contributions are negligible. Furthermore, Fig. 16(b) shows the effective ground state energy difference

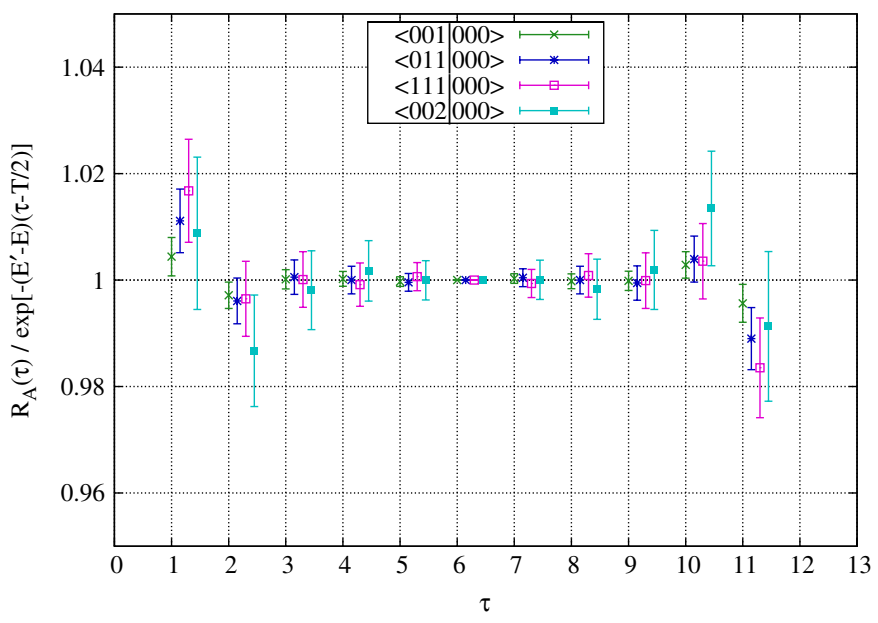

(a)

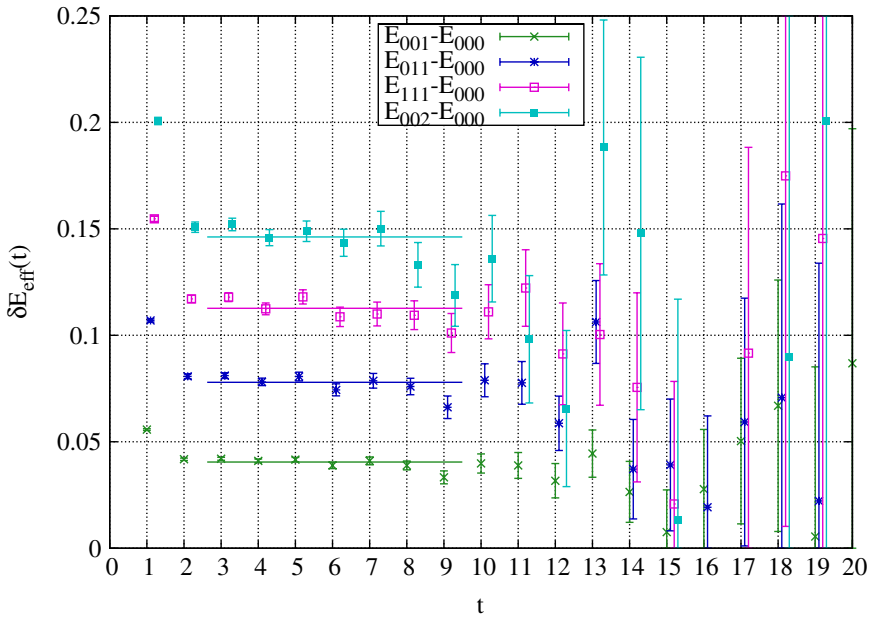

(b)

FIG. 16 (color online). Panel (a) shows the ratio $A(\tau)$ in Eq. (80). Panel (b) shows the effective energy difference (81) in lattice units and the fit values of $E^{\prime}-E$ used in panel (a). The degree to which the contaminations to all $\vec{P}^{\prime} \neq \overrightarrow{0}$ two-point correlators are canceled by the contamination to the $\vec{P}=\overrightarrow{0}$ correlator is remarkable. 


$$
\delta E^{\mathrm{eff}}(t)=\log \left[\frac{C_{2 \mathrm{pt}}\left(t, P^{\prime}\right)}{C_{2 \mathrm{pt}}\left(t+1, P^{\prime}\right)} / \frac{C_{2 \mathrm{pt}}(t, P)}{C_{2 \mathrm{pt}}(t+1, P)}\right],
$$

which in the absence of any excited state contaminants, would simply be $\delta E^{\mathrm{eff}}(t)=\left(E^{\prime}-E\right)$. For comparison, the values of $E^{\prime}-E$ determined above are plotted on the same graph, and agree nicely in the fiducial range $2 \leq \tau \leq 10$. Thus, we neglect small contaminations from this factor.

Second, we estimate the contribution to $R_{N}$ defined in Eq. (21) assuming only one excited state and no oscillating term ${ }^{14}$.

$$
\begin{aligned}
& \left.C_{3 \mathrm{pt}}(\tau, T) \approx C_{3 \mathrm{pt}}(\tau, T)\right|_{0}\left[1+\sqrt{\frac{Z_{1}}{Z_{0}}} \frac{\mathcal{O}_{0^{\prime} 1}}{\mathcal{O}_{0^{\prime} 0}} e^{-\Delta E \tau}+\sqrt{\frac{Z_{1}^{\prime}}{Z_{0}^{\prime}}} \frac{\mathcal{O}_{1^{\prime} 0}}{\mathcal{O}_{0^{\prime} 0}} e^{-\Delta E^{\prime}(T-\tau)}+\sqrt{\frac{Z_{1}^{\prime} Z_{1}}{Z_{0}^{\prime} Z_{0}}} \frac{\mathcal{O}_{1^{\prime} 1}}{\mathcal{O}_{0^{\prime} 0}} e^{-\Delta E^{\prime}(T-\tau)-\Delta E \tau}\right], \\
& \frac{C_{3 \mathrm{pt}}(\tau, T)}{\sqrt{C_{2 \mathrm{pt}}(T) C_{2 \mathrm{pt}}^{\prime}(T)}} \approx\left(\frac{C_{3 \mathrm{pt}}(\tau, T)}{\sqrt{C_{2 \mathrm{pt}}(T) C_{2 \mathrm{pt}}^{\prime}(T)}}\right)_{0} \times\left[1+\frac{\mathcal{O}_{0^{\prime} 1}}{\mathcal{O}_{0^{\prime} 0}} \delta R_{10}(\tau)+\frac{\mathcal{O}_{1^{\prime} 0}}{\mathcal{O}_{0^{\prime} 0}} \delta R_{10}^{\prime}(T-\tau)+\frac{\mathcal{O}_{1^{\prime} 1}}{\mathcal{O}_{0^{\prime} 0}} \delta R_{10}(\tau) \delta R_{10}^{\prime}(T-\tau)\right. \\
& \left.-\frac{1}{2}\left(\delta R_{11}+\delta R_{11}^{\prime}\right)\right]
\end{aligned}
$$

where

$$
\begin{aligned}
\delta R_{10}^{(\prime)}(\tau) & =\sqrt{\frac{Z_{1}^{(\prime)}}{Z_{0}^{(\prime)}}} e^{-\Delta E^{(\prime)} \tau}, \\
\delta R_{11}^{(\prime)} & =\frac{Z_{1}^{(\prime)}}{Z_{0}^{(\prime)}} e^{-\Delta E^{(\prime)} T}=\left[\delta R_{10}^{(\prime)}(T / 2)\right]^{2},
\end{aligned}
$$

and we have expanded to leading order assuming that $\delta R_{11}^{(\prime)} \ll 1$. The value of the suppression factor $\delta R_{10}^{(\prime)}(\tau)$ is shown in Fig. 17. Its values are estimated from the fit parameters $Z_{0,1}, E_{1,0}$ in Eq. (79), and the errors are computed using the jackknife procedure. Note that $\delta R_{10}^{(\prime)}(\tau)$ falls off steeply with $\tau$. As a result, its contribution can

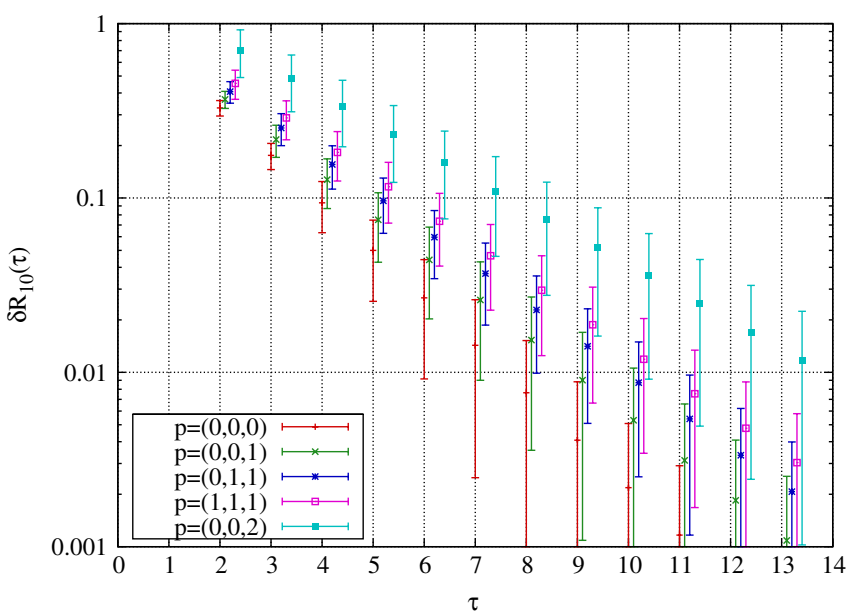

FIG. 17 (color online). Suppression factor for the excited state contributions $\delta R_{10}(\tau)(83)$, as estimated from fitting the twopoint function. Note that the actual matrix element $\mathcal{O}_{10}$ is not included in the plotted value, which therefore shows only the relative fall-off of the exponential tail contamination. Note also that the factor for the $p=(0,0,2)$ state is substantially larger than for the other states shown. be easily detected and removed by fitting the plateau with

$$
R^{\mathcal{O}}(\tau) \approx C_{0}+C_{1} e^{-\Delta E \tau}+C_{1}^{\prime} e^{-\Delta E^{\prime}(T-\tau)} .
$$

From Fig. 17 one may estimate the last two terms in the contamination formula (82), suppressed by $\delta R_{11}^{(\prime)}$ and $\delta R_{10}(\tau) \delta R_{10}^{\prime}(T-\tau)$. If one further assumes that the excited state matrix elements are at most of the same order as the ground state elements, $\frac{\mathcal{O}_{1^{\prime} 1}}{\mathcal{O}_{l^{\prime}}} \lesssim 1$, the effect of the last two terms in Eq. (82) is well below 1\%. It is also worth noting that higher momentum matrix elements with $\vec{p}=$ $(0,0,2)$ would contain substantially larger contamination, as compared to lower momenta. Such matrix elements are excluded from our analysis.

Finally, we compare the form factors extracted using the plateau average with those from fitting the $\tau$ dependence to Eq. (84). Because of the uncertainty in the two-point correlator fitting parameters, we perform fits for a range of mass gaps $\Delta M_{N}=0.4,0.6$ and 0.8 , which bracket the fitted values from different fitting ranges and fitting with or without the oscillating term in Eq. (79). The energy gaps $\Delta E$ for the $\vec{P} \neq 0$ states are computed using the continuum dispersion formula. The result is statistically independent of the mass gap value used (see Fig. 18) and is stable when fitting inside the region $2 \leq \tau \leq 10$. The complete consistency between conventional plateau averages and results for which excited state contaminants are explicitly included in the analysis and separated from the physical ground state contribution clearly indicates the absence of systematic errors from excited state contaminants in our present results.

In addition, we have also compared results with two different source-sink separations, $T=12$ and $T=14$. If the coherent sink technique were ever to introduce addi-

\footnotetext{
${ }^{14}$ We neglect the contribution of oscillating states because they decay even faster than excited states.
} 


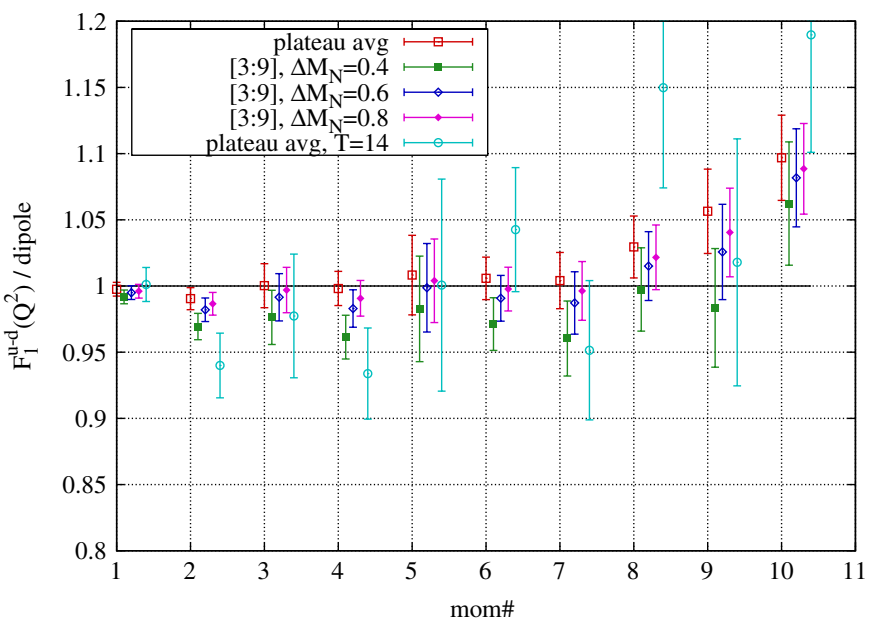

(a)

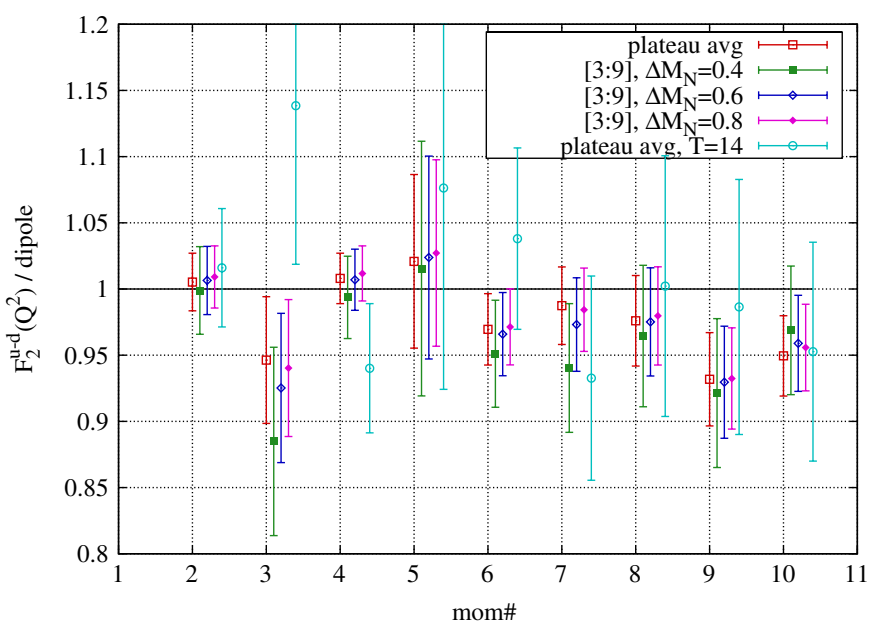

(b)

FIG. 18 (color online). Comparison of the isovector Dirac (a) and Pauli (b) form factors extracted from plateau averages and from fitting plateaus with formula (84) for the ensemble with the lightest pion mass $m_{\pi}=297 \mathrm{MeV}$. The result is stable with variation of the mass gap $\Delta M_{N}$, which means that the contamination is small. All but the last group of points use source-sink separation $T=12$. The last group, calculated for 330 gauge configurations, uses the larger separation $T=14$ and independent backward propagators. Each form factor value is divided by the central value of the dipole fit. Table II lists the momentum combinations corresponding to each index on the horizontal axis.

tional noise into the calculation, one would expect it to be worse for the larger $T$, for which the first adjacent unwanted sink is closer. Hence, in the case of $T=14$, we have used independent sinks to check that this is not a problem. One of the typical plateaus comparing $T=12$ and $T=14$ separations is plotted in Fig. 19 and shows agreement within statistics. Separations 12 and 14 are also compared in Fig. 18, where we show each of the form factors computed on a subset of the $a m_{q}=0.004$ ensemble using independent backward propagators and the larger source-sink separation $T=14$. The agreement of results

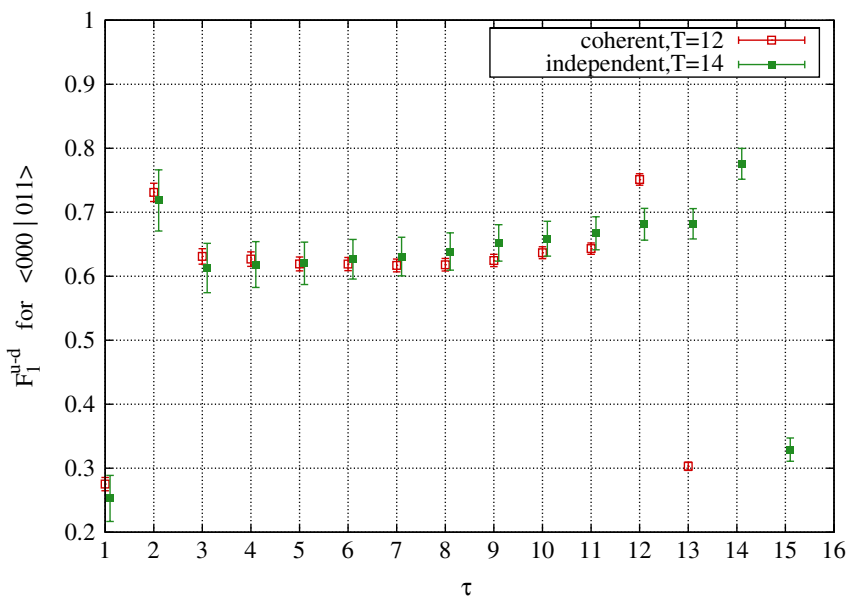

FIG. 19 (color online). Comparison of $F_{1}^{u-d}$ plateau using coherent backward propagators with $T=12$ and independent backward propagators with $T=14$. The momentum transfer $Q^{2}$ corresponds to $\langle 000 \mid 011\rangle$. that use two different separations and techniques directly indicates that our method does not suffer from the systematic effects due to excited states or the coherent propagator technique.

\section{B. Finite-volume dependence}

Ideally, we would like to control systematic errors arising from volume dependence using an effective field theory that describes the dependence of the observables of interest as a function of spatial volume and pion mass, and a set of calculations of the lattice observables with a specified action for a range of volumes at pion masses for which the effective field theory is applicable. Verifying that the effective theory fits the measured volume dependence with low-energy constants that are consistent with other lattice and phenomenological constraints would then assure solid theoretical and computational control of finite-volume effects. To date, this program has not been carried out completely for form factors with any lattice action, and finite-volume effects have been a convenient excuse for any disagreements with experiment. Hence, it is useful to examine the available data for domain wall fermions and to assess what quantitative evidence there is for or against significant finite-volume corrections.

To examine volume dependence carefully, it is important to only compare lattice calculations at different volumes that use precisely the same action and computational methodology. In this context, we believe it can be seriously misleading to argue on the basis of plots containing a variety of calculations with different actions, analysis techniques, renormalization schemes, etc. However, due to the 
high computational cost of domain wall fermions, the only high precision calculations of nucleon observables with two large volumes for light quarks that we are aware of are the mixed-action calculations for $355 \mathrm{MeV}$ pion mass in volumes of spatial extent $2.5 \mathrm{fm}$ and $3.5 \mathrm{fm}$, corresponding to $m_{\pi} L=4.4$ and 6.2 , respectively [26,27]. Thus, we will base our arguments on these results.

In order to make a consistent comparison, we calculate the form factor slopes at zero momentum transfer by taking the most accurate results at the smallest momentum transfer values $\left(\frac{2 \pi}{L}, 0,0\right),(0,0,0)$ on each lattice and using the one-parameter dipole formula (28) to determine the slope at the origin. Comparing the results for $r_{1}^{v}$ and quadratically combining the errors for the two independent calculations, we find that the fractional difference between $r_{1}^{v}$ at $3.5 \mathrm{fm}$ and $2.5 \mathrm{fm}$ is $(3.7 \pm 2.6) \%$ at $m_{\pi}=355 \mathrm{MeV} .{ }^{15}$ Further evidence suggesting finite-volume corrections to radii are small is the fact that in Ref. [29], even decreasing the lattice size to $1.8 \mathrm{fm}$ yields small changes in $r_{1}^{v}$ and $r_{2}^{v}$. At the same time, it cannot be excluded with the present data that rapidly growing finite-size effects below $m_{\pi}=$ $355 \mathrm{MeV}$ are affecting the pion-mass dependence of $r_{1}^{v}$ in our calculations. This will have to be resolved in the future.

\section{COMPARISON WITH PREVIOUS CALCULATIONS}

We briefly compare our results and conclusions with those of previous calculations. We start with the isovector Dirac and Pauli radii, $r_{1}^{v}$ and $r_{2}^{v}$. Previous calculations using Wilson fermions had reached pion masses of about $400 \mathrm{MeV}$. Both the quenched [16,17] and $N_{f}=2$ unquenched results [17] showed a mild pion-mass dependence for $r_{1}^{v}$ and $r_{2}^{v}$. A recent calculation on $N_{f}=2+1$ domain wall fermion configurations at $a=0.114 \mathrm{fm}$ extended the range of pion masses down to $330 \mathrm{MeV}$ [29]. These results show that the very mild upward trend of $r_{1}^{v}$ and $r_{2}^{v}$ extends down to that pion mass. Summary plots comparing results for Wilson fermions with zero and two flavors and domain wall fermions for zero, two, and $2+1$ flavors are given in Figs. 14 and 19 of Ref. [29]. For the case of $r_{1}^{v}$, which has smaller statistical errors, for each action, the data tend to lie on straight lines with comparable small slope and some scatter in normalization, with perhaps a hint that the $N_{f}=2$ calculations, performed on box sizes $1.9 \mathrm{fm}$, lie somewhat low. The $r_{2}^{v}$ data also appear to lie on straight lines with similar small slope, albeit with larger scatter. Our results for $r_{1}^{v}$ and $r_{2}^{v}$, which extend down to $m_{\pi}=300 \mathrm{MeV}$, also show a small pion-mass depen-

\footnotetext{
${ }^{15}$ We note that as discussed in Ref. [27], dipole fits of all the form factor data out to some fixed cutoff yield discrepancies in the dipole fits between the two volumes that increase as the cutoff increases, but this comparison focusses on other features of the form factor besides the radius that we seek to chirally extrapolate.
}

dence, and are consistent within statistical errors with the $2+1$ flavor domain wall results on $a=0.114$ fm lattices. We conclude that this flat behavior, surprising as it is from the chiral effective theory point of view, is genuine. The one-loop SSE formulas of Sec. III C 1 cannot accommodate this "flat" pion-mass dependence in the radii down to such low values of the pion mass $\sim 300 \mathrm{MeV}$, with or without the inclusion of a higher-order core term. Indeed, the curves shown in Figs. 8(a) and 8(b) indicate that the SSE calculation would have favored an upward trend in the extracted isovector radii which should have become visible in the pion-mass range studied in this work, consistent with the expectations drawn in Ref. [16]. The only explanation for this behavior available at the moment is that the leading one-loop SSE calculation is only valid for pion masses $<300 \mathrm{MeV}$.

As for the anomalous magnetic moment $\kappa_{v}$, our results are in very good agreement with those obtained with $N_{f}=$ 2 dynamical Wilson fermions in [21], with recent $N_{f}=2$ twisted-mass results [23] and with the recent $N_{f}=2+1$ domain wall calculation [29]. We remark that our Fig. 8 displays the normalized anomalous magnetic moment $\kappa^{\text {norm }}$, while Fig. 17 of Ref. [29] shows the magnetic moment normalized by the quark-mass dependent nucleon mass, $\kappa^{\text {lat }}$. The difference between the two figures ${ }^{16}$ reflects the $m_{\pi}$ dependence of the nucleon mass, which is quite strong (see Fig. 9). All in all, for $\kappa_{v}$ too, the calculated pion-mass dependence is rather mild, and results at lower pion masses will have to bend upwards rather sharply if they are to agree with the experimental value.

\section{SUMMARY AND CONCLUSIONS}

We have presented lattice calculations of nucleon form factors with $N_{f}=2+1$ flavors of dynamical domain wall fermions on fine $32^{3} \times 64$ lattices with $a=0.084 \mathrm{fm}$ at pion masses of 297, 355, and $403 \mathrm{MeV}$ that achieve a new level of precision in both statistical and systematic errors. Statistical errors have been reduced by using from 3600 to 7064 measurements of operators at a given mass by performing 8 measurements per lattice and verifying their statistical independence. Statistical errors and error correlations have been carefully analyzed in our overdetermined analysis, which combines as many stochastically distinct measurements of the same physical form factors as practical.

Because of the high level of statistical precision, we have carefully investigated and controlled potential sources of systematic error. We have ruled out systematic errors arising from the source-sink separation in two different ways. First, we have derived analytic expressions for the contamination by excited states, and, using lattice data from two-point correlation functions, have shown quanti-

\footnotetext{
${ }^{16}$ The numerical difference between the lattice data and the experimental value is smaller in the case of $\kappa_{\text {lat }}$.
} 
tatively that the coefficients of excited state admixtures in these expressions yield negligible contributions to the observables of interest. In addition, we compared explicit calculations with source-sink separations $T=12$ and $T=$ 14 and have shown that results from the two source-sink separations are indeed statistically consistent, as expected from the excited state analysis. We have verified that even in the worst case- the lightest pion mass and maximum source-sink separation-results calculated using the timesaving coherent sink technique are consistent with results calculated with conventional independent sinks. By comparison with companion calculations on a coarse lattice with $a=0.114 \mathrm{fm}$, we have verified that lattice spacing errors are small. Finally, we argue that finite-volume corrections to the present calculations in a volume of spatial extent $2.5 \mathrm{fm}$ are small, since the fractional change in $r_{1}^{v}$ in the recent mixed-action calculations with two volumes is $3.7 \pm 2.6 \%$, and our present results are consistent with that calculation.

The high precision of the calculated form factors is shown in Figs. 4 and 5, where, in order to see the discrepancies with dipole fits, we plotted the ratio of the lattice calculations to the best dipole fits on an expanded scale. This precise data enabled us to extract the Dirac radius, $r_{1}^{v}$, Pauli radius $r_{2}^{v}$, and anomalous magnetic moment $\kappa_{v}$ with much smaller errors than in earlier calculations and to study chiral extrapolations to correspondingly higher precision. In contrast to earlier studies in which the lattice error bars were sufficiently large that the data appeared to be consistent with chiral perturbation theory, in this work we have shown that the $m_{\pi}$ dependence of the lattice results for $\left(r_{1}^{v}\right)^{2},\left(r_{2}^{v}\right)^{2}$, and $\kappa_{v}$ at the three masses 297, 355 , and $403 \mathrm{MeV}$ cannot be simultaneously fit by either $\mathcal{O}\left(\epsilon^{3}\right)$ SSE or NNLO BChPT. The data points for $\left(r_{1}^{v}\right)^{2}$ rise too slowly with decreasing $m_{\pi}$ and the data for $\left(r_{2}^{v}\right)^{2}$ are too flat to be fit by either the SSE or BChPT curves that rise smoothly with decreasing $m_{\pi}$ to approach the experimental results. Since there happen to be three free parameters in SSE to fit the 3 measured values of $\kappa_{v}$, the SSE can actually fit the anomalous magnetic moment, but BChPT, which is physically constrained to rise with decreasing $m_{\pi}$, is also seriously in conflict with the lattice measurements of $\kappa_{v}$ at the accessible masses. Similarly, we were unable to simultaneously fit the isoscalar quantities $\left(r_{1,2}^{s}\right)^{2}$ and $\kappa_{s}$, which, to this order of ChPT, have fewer parameters.

With the present data at these three pion masses, we see three possible explanations for the discrepancy with chiral perturbation theory. One possibility is an outright error somewhere in the lattice calculations. However, by virtue of meticulous checks, key calculations with independent codes, and the qualitative similarity of our results to those of other groups [17,21,23,28,29], we believe this is unlikely. A second possibility is that finite-volume effects are significantly larger than the estimates we obtained from our $355 \mathrm{MeV}$ mixed-action studies for spatial sizes $2.5 \mathrm{fm}$ and $3.5 \mathrm{fm}$. This possibility clearly warrants further study of chiral perturbation theory for off-forward matrix elements in a finite-volume and careful high-statistics studies in a series of volumes. The third possibility is that chiral perturbation theory at the present order is not applicable for this range of $m_{\pi}$. Indeed, significant problems have previously been encountered in describing the $m_{\pi}$ dependence of baryon masses, and one observes, for example, that the highly linear dependence of the nucleon mass on $m_{\pi}$ seen in a variety of lattice calculations with different actions can only arise from an apparently unnatural cancellation of analytic and nonanalytic terms in chiral perturbation theory [54]. This possibility clearly warrants lattice calculations at a series of lower values of $m_{\pi}$ all the way down to the physical pion mass.

The last two possibilities each raise very interesting and important questions in hadron structure. Given the high computational cost of chiral fermions relative to improved Wilson fermions and the fact that there are no crucial operator mixing problems in form factors necessitating exact chiral symmetry on the lattice, it appears that the most expeditious means of understanding the volume dependence and behavior down to the physical pion mass will be with an appropriate form of an improved isotropic Wilson action. Such calculations are clearly essential for further progress in understanding the fundamental structure of the nucleon.

\section{ACKNOWLEDGMENTS}

This work is supported in part by the U. S. Department of Energy under Grants No. DE-FG02-94ER40818, No. DE-FG02-05ER25681, and No. DE-FG0296ER40965. Ph. H. acknowledges support by the EmmyNoether program and the cluster of excellence "Origin and Structure of the Universe" of the DFG, M. P. acknowledges support by the Alexander von Humboldt Foundation, and T. R. H. is supported by DFG via Grant No. SFB/TR 55. W. S. wishes to thank the Institute of Physics at Academia Sinica for their kind hospitality and support, as well as Jiunn-Wei Chen at National Taiwan University and Hsiang-nan Li at Academia Sinica for their hospitality and for valuable physics discussions and suggestions. Computations for this work were carried out using the Argonne Leadership Computing Facility at Argonne National Laboratory, which is supported by the Office of Science of the U. S. Department of Energy under Contract No. DE-AC02-06CH11357; using facilities of the USQCD Collaboration, which are funded by the Office of Science of the U. S. Department of Energy; and using resources provided by the New Mexico Computing Applications Center (NMCAC) on Encanto. The authors also wish to acknowledge use of dynamical domain wall configurations and universal propagators calculated by the RBC and LHPC Collaborations and the use of Chroma [74] SciDAC software. 


\section{APPENDIX A: FORM FACTOR TABLES}

We collect the results for the nucleon Dirac and Pauli form factors for individual flavors and flavor combinations for the three fine lattice spacing ensembles in Tables XIII, XIV, and XV. The dipole and tripole fit parameters of the isovector Dirac and Pauli form factors are collected in Tables XVI and XVII, respectively.

TABLE XIII. Renormalized results for the Dirac and Pauli form factors from the $a m_{l}=0.004$ ensemble with $m_{\pi} \approx 297 \mathrm{MeV}$.

\begin{tabular}{|c|c|c|c|c|c|c|c|c|c|}
\hline$(a Q)^{2}$ & $Q^{2}\left[\mathrm{GeV}^{2}\right]$ & $F_{1}^{u}$ & $F_{1}^{d}$ & $F_{1}^{u+d}$ & $F_{1}^{u-d}$ & $F_{2}^{u}$ & $F_{2}^{d}$ & $F_{2}^{u+d}$ & $F_{2}^{u-d}$ \\
\hline 0.000000 & 0.000 & $2.004(5)$ & $1.004(3)$ & $3.008(7)$ & $1.000(5)$ & & & & \\
\hline 0.037025 & 0.203 & $1.457(7)$ & $0.683(4)$ & $2.140(9)$ & $0.774(7)$ & $1.050(46)$ & $-1.092(27)$ & $-0.042(59)$ & $2.142(46)$ \\
\hline 0.037235 & 0.204 & $1.462(13)$ & $0.681(8)$ & $2.142(17)$ & $0.781(13)$ & $0.956(106)$ & $-1.056(60)$ & $-0.100(139)$ & $2.013(102)$ \\
\hline 0.071421 & 0.392 & $1.132(9)$ & $0.497(5)$ & $1.629(12)$ & $0.635(8)$ & $0.853(35)$ & $-0.822(23)$ & $0.031(50)$ & $1.675(32)$ \\
\hline 0.072155 & 0.396 & $1.130(21)$ & $0.491(12)$ & $1.621(29)$ & $0.639(19)$ & $0.995(104)$ & $-0.693(55)$ & $0.302(127)$ & $1.688(108)$ \\
\hline 0.077106 & 0.423 & $1.089(11)$ & $0.470(6)$ & $1.560(15)$ & $0.619(10)$ & $0.745(42)$ & $-0.806(26)$ & $-0.061(54)$ & $1.550(43)$ \\
\hline 0.103678 & 0.569 & $0.912(13)$ & $0.376(7)$ & $1.288(18)$ & $0.536(11)$ & $0.685(44)$ & $-0.647(26)$ & $0.038(61)$ & $1.332(39)$ \\
\hline 0.114341 & 0.627 & $0.870(13)$ & $0.350(7)$ & $1.219(17)$ & $0.520(12)$ & $0.634(41)$ & $-0.601(26)$ & $0.034(54)$ & $1.235(43)$ \\
\hline 0.154213 & 0.846 & $0.696(15)$ & $0.256(8)$ & $0.951(20)$ & $0.440(13)$ & $0.479(36)$ & $-0.463(23)$ & $0.016(49)$ & $0.943(36)$ \\
\hline 0.191447 & 1.050 & $0.591(13)$ & $0.204(7)$ & $0.795(17)$ & $0.387(11)$ & $0.408(25)$ & $-0.388(16)$ & $0.020(34)$ & $0.796(25)$ \\
\hline
\end{tabular}

TABLE XIV. Renormalized results for Dirac and Pauli form factors from the $a m_{l}=0.006$ ensemble with $m_{\pi} \approx 355 \mathrm{MeV}$.

\begin{tabular}{|c|c|c|c|c|c|c|c|c|c|}
\hline$(a Q)^{2}$ & $Q^{2}\left[\mathrm{GeV}^{2}\right]$ & $F_{1}^{u}$ & $F_{1}^{d}$ & $F_{1}^{u+d}$ & $F_{1}^{u-d}$ & $F_{2}^{u}$ & $F_{2}^{d}$ & $F_{2}^{u+d}$ & $F_{2}^{u-d}$ \\
\hline 0.000000 & 0.000 & $2.000(3)$ & $1.000(2)$ & $2.999(4)$ & $1.000(3)$ & & & & \\
\hline 0.037176 & 0.204 & $1.478(4)$ & $0.691(2)$ & $2.169(6)$ & $0.788(4)$ & $1.132(31)$ & $-1.192(17)$ & $-0.061(40)$ & $2.324(30)$ \\
\hline 0.037348 & 0.205 & $1.471(8)$ & $0.691(4)$ & $2.162(10)$ & $0.779(8)$ & $1.226(71)$ & $-1.130(38)$ & $0.096(93)$ & $2.356(66)$ \\
\hline 0.071948 & 0.395 & $1.162(6)$ & $0.509(3)$ & $1.671(8)$ & $0.653(5)$ & $0.909(25)$ & $-0.923(15)$ & $-0.014(34)$ & $1.832(24)$ \\
\hline 0.072559 & 0.398 & $1.155(12)$ & $0.503(7)$ & $1.659(17)$ & $0.652(11)$ & $0.866(62)$ & $-0.875(33)$ & $-0.009(79)$ & $1.741(59)$ \\
\hline 0.077106 & 0.423 & $1.134(8)$ & $0.491(4)$ & $1.625(10)$ & $0.643(7)$ & $0.878(28)$ & $-0.870(16)$ & $0.008(36)$ & $1.748(28)$ \\
\hline 0.104730 & 0.574 & $0.946(9)$ & $0.389(5)$ & $1.336(12)$ & $0.557(7)$ & $0.745(28)$ & $-0.727(17)$ & $0.018(37)$ & $1.472(27)$ \\
\hline 0.114455 & 0.628 & $0.904(9)$ & $0.365(4)$ & $1.269(11)$ & $0.540(8)$ & $0.693(29)$ & $-0.656(16)$ & $0.036(37)$ & $1.349(28)$ \\
\hline 0.154213 & 0.846 & $0.736(10)$ & $0.285(5)$ & $1.021(14)$ & $0.452(9)$ & $0.560(25)$ & $-0.505(15)$ & $0.056(33)$ & $1.065(25)$ \\
\hline 0.191561 & 1.051 & $0.619(9)$ & $0.225(4)$ & $0.844(11)$ & $0.393(7)$ & $0.476(17)$ & $-0.407(11)$ & $0.070(23)$ & $0.883(17)$ \\
\hline
\end{tabular}

TABLE XV. Renormalized results for Dirac and Pauli form factors from the $a m_{l}=0.008$ ensemble with $m_{\pi} \approx 403 \mathrm{MeV}$.

\begin{tabular}{|c|c|c|c|c|c|c|c|c|c|}
\hline$(a Q)^{2}$ & $Q^{2}\left[\mathrm{GeV}^{2}\right]$ & $F_{1}^{u}$ & $F_{1}^{d}$ & $F_{1}^{u+d}$ & $F_{1}^{u-d}$ & $F_{2}^{u}$ & $F_{2}^{d}$ & $F_{2}^{u+d}$ & $F_{2}^{u-d}$ \\
\hline 0.000000 & 0.000 & $2.006(3)$ & $1.006(1)$ & $3.012(3)$ & $1.000(2)$ & & & & \\
\hline 0.037277 & 0.204 & $1.502(4)$ & $0.706(2)$ & $2.208(5)$ & $0.796(4)$ & $1.210(32)$ & $-1.193(19)$ & $0.016(43)$ & $2.403(31)$ \\
\hline 0.037427 & 0.205 & $1.499(8)$ & $0.706(4)$ & $2.204(11)$ & $0.793(7)$ & $1.342(65)$ & $-1.131(39)$ & $0.211(85)$ & $2.473(65)$ \\
\hline 0.072306 & 0.397 & $1.180(6)$ & $0.521(4)$ & $1.702(8)$ & $0.659(5)$ & $0.965(26)$ & $-0.926(17)$ & $0.038(36)$ & $1.891(26)$ \\
\hline 0.072839 & 0.400 & $1.168(12)$ & $0.519(6)$ & $1.687(16)$ & $0.649(10)$ & $0.985(60)$ & $-0.922(36)$ & $0.063(80)$ & $1.908(59)$ \\
\hline 0.077106 & 0.423 & $1.160(8)$ & $0.508(4)$ & $1.667(11)$ & $0.652(7)$ & $0.918(29)$ & $-0.861(19)$ & $0.058(39)$ & $1.779(29)$ \\
\hline 0.105450 & 0.578 & $0.965(8)$ & $0.401(5)$ & $1.366(11)$ & $0.564(7)$ & $0.783(29)$ & $-0.749(18)$ & $0.035(39)$ & $1.532(28)$ \\
\hline 0.114533 & 0.628 & $0.924(8)$ & $0.381(5)$ & $1.305(11)$ & $0.543(8)$ & $0.710(27)$ & $-0.669(18)$ & $0.041(38)$ & $1.379(26)$ \\
\hline 0.154213 & 0.846 & $0.771(11)$ & $0.302(6)$ & $1.073(14)$ & $0.469(9)$ & $0.567(24)$ & $-0.539(16)$ & $0.028(33)$ & $1.106(24)$ \\
\hline 0.191639 & 1.051 & $0.633(9)$ & $0.234(5)$ & $0.867(12)$ & $0.399(8)$ & $0.459(17)$ & $-0.442(12)$ & $0.017(24)$ & $0.901(17)$ \\
\hline
\end{tabular}


TABLE XVI. Comparison of fit Ansätze to the isovector Dirac form factors $F_{1}^{u-d}$ for all three ensembles with different $Q^{2}$ cutoffs.

\begin{tabular}{|c|c|c|c|c|}
\hline \multicolumn{5}{|c|}{$a m_{l}=0.004$} \\
\hline & \multicolumn{2}{|c|}{ Dipole } & \multicolumn{2}{|c|}{ Tripole } \\
\hline$Q^{2}$ cutoff $\left[\mathrm{GeV}^{2}\right]$ & $\chi^{2} /$ dof & $M_{D}^{-2}\left[\mathrm{GeV}^{-2}\right]$ & $\chi^{2} /$ dof & $M_{T}^{-2}\left[\mathrm{GeV}^{-2}\right]$ \\
\hline 0.3 & $0.2(6)$ & $0.670(22)$ & $0.2(6)$ & $0.436(14)$ \\
\hline 0.4 & $0.3(6)$ & $0.659(19)$ & $0.8(9)$ & $0.424(12)$ \\
\hline 0.5 & $0.5(6)$ & $0.653(17)$ & $1.0(9)$ & $0.418(11)$ \\
\hline 0.6 & $0.4(5)$ & $0.652(17)$ & $1.0(8)$ & $0.417(11)$ \\
\hline 0.7 & $0.5(5)$ & $0.649(17)$ & $1.2(8)$ & $0.414(11)$ \\
\hline 0.9 & $0.9(7)$ & $0.638(16)$ & $1.9(1.0)$ & $0.404(10)$ \\
\hline 1.1 & $1.4(8)$ & $0.632(16)$ & $3.0(1.1)$ & $0.398(10)$ \\
\hline \multicolumn{5}{|c|}{$a m_{l}=0.006$} \\
\hline & \multicolumn{2}{|c|}{ Dipole } & \multicolumn{2}{|c|}{ Tripole } \\
\hline$Q^{2}$ cutoff $\left[\mathrm{GeV}^{2}\right]$ & $\chi^{2} /$ dof & $M_{D}^{-2}\left[\mathrm{GeV}^{-2}\right]$ & $\chi^{2} /$ dof & $M_{T}^{-2}\left[\mathrm{GeV}^{-2}\right]$ \\
\hline 0.3 & $0.5(1.0)$ & $0.625(13)$ & $0.5(1.0)$ & $0.407(8)$ \\
\hline 0.4 & $1.8(1.3)$ & $0.610(12)$ & $3.3(1.8)$ & $0.393(7)$ \\
\hline 0.5 & $2.8(1.5)$ & $0.602(11)$ & $4.8(1.9)$ & $0.386(7)$ \\
\hline 0.6 & $2.3(1.2)$ & $0.602(11)$ & $4.2(1.7)$ & $0.386(7)$ \\
\hline 0.7 & $2.1(1.1)$ & $0.601(11)$ & $3.8(1.5)$ & $0.385(7)$ \\
\hline 0.9 & $2.0(1.0)$ & $0.597(11)$ & 4.1(1.4) & $0.379(7)$ \\
\hline \multirow[t]{3}{*}{1.1} & $2.0(9)$ & $0.595(11)$ & $4.8(1.5)$ & $0.375(7)$ \\
\hline & \multicolumn{4}{|c|}{$a m_{l}=0.008$} \\
\hline & \multicolumn{2}{|c|}{ Dipole } & \multicolumn{2}{|c|}{ Tripole } \\
\hline$Q^{2}$ cutoff $\left[\mathrm{GeV}^{2}\right]$ & $\chi^{2} /$ dof & $M_{D}^{-2}\left[\mathrm{GeV}^{-2}\right]$ & $\chi^{2} /$ dof & $M_{T}^{-2}\left[\mathrm{GeV}^{-2}\right]$ \\
\hline 0.3 & $0.09(42)$ & $0.592(13)$ & $0.09(42)$ & $0.386(8)$ \\
\hline 0.4 & $0.3(5)$ & $0.588(12)$ & $1.0(1.0)$ & $0.380(7)$ \\
\hline 0.5 & $0.9(9)$ & $0.582(11)$ & $1.9(1.2)$ & $0.374(7)$ \\
\hline 0.6 & $1.0(8)$ & $0.579(11)$ & $2.2(1.2)$ & $0.371(7)$ \\
\hline 0.7 & $0.9(7)$ & $0.579(11)$ & $2.0(1.1)$ & $0.370(7)$ \\
\hline 0.9 & $1.1(7)$ & $0.575(10)$ & $2.7(1.2)$ & $0.366(6)$ \\
\hline 1.1 & $1.0(7)$ & $0.575(10)$ & $2.7(1.1)$ & $0.365(6)$ \\
\hline
\end{tabular}

\section{APPENDIX B: SMEARED NUCLEON SOURCES FOR DOMAIN WALL FERMIONS}

Since careful optimization of the interpolating field for the nucleon source is crucial for the high precision calculations described in this work, in this appendix we describe in detail our optimization procedure and record the optimal parameters in two commonly used conventions.

We have two objectives in constructing sources for propagators that will be optimal for calculating hadronic matrix elements. The first is to maximize the overlap between the interpolating field acting on the QCD vacuum and the hadronic ground state. The second is to minimize fluctuations arising from the source itself. Let $\bar{N}$ denote an interpolating field with the quantum numbers of the hadron, $|\Psi\rangle=\mathcal{C}^{-1 / 2} \bar{N}|\Omega\rangle$ denote the normalized state obtained by its action on the vacuum, and $|n\rangle$ denote the $n$th eigenstate of the hadron (projected to zero momentum in the present discussion). Then, maximizing $|\langle 0 \mid \Psi\rangle|^{2}$ minimizes the contributions of excited states to the measurement of the hadronic matrix element of an operator $\mathcal{O}$

$$
\begin{aligned}
\left\langle N\left(t_{3}\right) \mathcal{O}\left(t_{2}\right) N\left(t_{1}\right)\right\rangle= & \mathcal{C} \sum_{n, m}\langle\Psi \mid n\rangle\langle n|\mathcal{O}| m\rangle \\
& \times\langle m \mid \Psi\rangle e^{-E_{n}\left(t_{3}-t_{2}\right)-E_{m}\left(t_{2}-t_{1}\right)},
\end{aligned}
$$

and hence enables one to reduce the source-sink separation while controlling contamination from excited states as discussed in Sec. VA.

The first objective is met by using smeared propagators and treating the rms radius of the smearing as a variational parameter. Although similar effects can be accomplished with gauge fixed sources, we use gauge invariant sources of the Wuppertal [38,39], or equivalently, Gaussian form by smearing a delta function source over the three spatial dimensions of the source time slice.

Wuppertal smearing of a point source at the origin of time slice $t$ is defined in the MIT USQCD software as

$$
\begin{aligned}
\psi(x, t)= & \left(1+\alpha \sum_{i=1}^{3}\left[U(x, i) \delta_{x+\hat{i}, y}+U^{\dagger}(x-\hat{i}, i) \delta_{x-\hat{i}, y}\right]\right)^{N} \\
& \times \delta_{y, 0},
\end{aligned}
$$


TABLE XVII. Comparison of fit Ansätze to the isovector Pauli form factors $F_{2}^{u-d}$ for all three ensembles with different $Q^{2}$ cutoffs.

\begin{tabular}{|c|c|c|c|c|c|c|}
\hline \multicolumn{7}{|c|}{$a m_{l}=0.004$} \\
\hline & \multicolumn{3}{|c|}{ Dipole } & \multicolumn{3}{|c|}{ Tripole } \\
\hline$Q^{2}$ cutoff $\left[\mathrm{GeV}^{2}\right]$ & $\chi^{2} /$ dof & $F_{2}(0)$ & $M_{D}^{-2}\left[\mathrm{GeV}^{-2}\right]$ & $\chi^{2} /$ dof & $F_{2}(0)$ & $M_{T}^{-2}\left[\mathrm{GeV}^{-2}\right]$ \\
\hline 0.5 & $1.2(1.3)$ & $2.89(12)$ & $0.820(70)$ & $1.2(1.3)$ & $2.85(11)$ & $0.505(40)$ \\
\hline 0.6 & $1.1(1.1)$ & $2.92(11)$ & $0.846(63)$ & $1.0(1.0)$ & $2.87(10)$ & $0.516(36)$ \\
\hline 0.7 & $0.9(8)$ & $2.93(11)$ & $0.847(60)$ & $0.8(8)$ & $2.87(10)$ & $0.513(33)$ \\
\hline 0.9 & $0.9(8)$ & $2.98(9)$ & $0.888(46)$ & $0.7(7)$ & $2.89(8)$ & $0.526(15)$ \\
\hline 1.1 & $0.8(7)$ & 2.97(9) & $0.881(41)$ & $0.9(7)$ & $2.85(8)$ & $0.509(21)$ \\
\hline \multicolumn{7}{|c|}{$a m_{l}=0.006$} \\
\hline & \multicolumn{3}{|c|}{ Dipole } & \multicolumn{3}{|c|}{ Tripole } \\
\hline$Q^{2}$ cut $\left[\mathrm{GeV}^{2}\right]$ & $\chi^{2} /$ dof & $F_{2}(0)$ & $M_{D}^{-2}\left[\mathrm{GeV}^{-2}\right]$ & $\chi^{2} /$ dof & $F_{2}(0)$ & $M_{T}^{-2}\left[\mathrm{GeV}^{-2}\right]$ \\
\hline 0.5 & $1.7(1.5)$ & $3.14(7)$ & $0.797(39)$ & $1.6(1.5)$ & $3.10(7)$ & $0.492(22)$ \\
\hline 0.6 & $1.4(1.2)$ & $3.16(7)$ & $0.810(35)$ & $1.2(1.1)$ & $3.10(6)$ & $0.495(20)$ \\
\hline 0.7 & $1.5(1.1)$ & $3.18(7)$ & $0.825(33)$ & $1.1(1.0)$ & $3.12(6)$ & $0.501(19)$ \\
\hline 0.9 & $1.4(1.0)$ & $3.22(6)$ & $0.851(26)$ & $1.0(8)$ & $3.13(5)$ & $0.505(14)$ \\
\hline 1.1 & $1.3(9)$ & $3.24(5)$ & $0.861(22)$ & $1.0(7)$ & $3.11(5)$ & $0.499(12)$ \\
\hline \multicolumn{7}{|c|}{$a m_{l}=0.008$} \\
\hline & \multicolumn{3}{|c|}{ Dipole } & \multicolumn{3}{|c|}{ Tripole } \\
\hline$Q^{2}$ cut $\left[\mathrm{GeV}^{2}\right]$ & $\chi^{2} /$ dof & $F_{2}(0)$ & $M_{D}^{-2}\left[\mathrm{GeV}^{-2}\right]$ & $\chi^{2} /$ dof & $F_{2}(0)$ & $M_{T}^{-2}\left[\mathrm{GeV}^{-2}\right]$ \\
\hline 0.5 & $2.2(1.7)$ & $3.26(7)$ & $0.813(34)$ & $2.1(1.7)$ & $3.21(6)$ & $0.501(19)$ \\
\hline 0.6 & $1.6(1.3)$ & $3.26(6)$ & $0.813(33)$ & $1.7(1.3)$ & $3.20(6)$ & $0.497(19)$ \\
\hline 0.7 & $2.5(1.4)$ & $3.29(6)$ & $0.841(31)$ & $2.1(1.3)$ & $3.22(6)$ & $0.511(17)$ \\
\hline 0.9 & $2.1(1.2)$ & $3.31(5)$ & $0.851(22)$ & $1.8(1.1)$ & $3.22(5)$ & $0.506(12)$ \\
\hline 1.1 & $2.0(1.1)$ & $3.32(5)$ & $0.862(20)$ & $1.6(1.0)$ & $3.21(5)$ & $0.502(10)$ \\
\hline
\end{tabular}

and Gaussian smearing is defined in CHROMA software as

$$
\begin{aligned}
\psi(x, t)= & \left(1-\frac{\sigma^{2} \nabla^{2}}{4 N}\right)^{N} \psi(x, t) \\
= & \left(1-\frac{3 \sigma^{2}}{2 N}\right)^{N}\left(1+\frac{\sigma^{2} / 4 N}{1-3 \sigma^{2} / 2 N}\right. \\
& \left.\times \sum_{i=1}^{3}\left[U(x, i) \delta_{x+\hat{i}, y}+U^{\dagger}(x-\hat{i}, i) \delta_{x-\hat{i}, y}\right]\right)^{N} \delta_{y, 0} .
\end{aligned}
$$

The CHROMA and MIT parameters are related by

$$
\begin{gathered}
\alpha=\frac{\sigma^{2} / 4 N}{1-3 \sigma^{2} / 2 N}, \\
\sigma^{2}=\frac{2 N \alpha}{3 \alpha+1 / 2} .
\end{gathered}
$$

Note that there is an instability for $\alpha<0$, since the sign of the source generated in Eq. (B2) is then $(-1)^{x+y+z}$, and the resulting spatially oscillating source has an extremely poor overlap with the physical ground state. In terms of the Gaussian parameters, the instability arises for $N<3 \sigma^{2} / 2$.

Because the smeared sources contain link variables $U$, the statistical fluctuations in correlation functions using these sources are larger than those arising from point sources. To attain our second objective of minimizing the fluctuations arising from the source itself, it is highly advantageous to perform APE smearing of the gauge links [40] used in generating the source on the time slice of the source. In each iteration of APE smearing, each link is replaced by a linear combination of itself and the sum of staples within that time slice, and projected back onto $S U(3)$ as follows,

$$
U_{x, i}^{(N+1)}=\operatorname{Proj}_{S U(3)}\left[U_{x, i}^{N}+\beta \sum_{j \neq i}^{3} U_{x, j}^{N} U_{x+j, i}^{N} U_{x+i, j}^{N \dagger}\right],
$$

and the APE smearing is repeated $N$ times. An alternative notation is

$$
U_{x, i}^{(N+1)}=\operatorname{Proj}_{S U(3)}\left[A U_{x, i}^{N}+\sum_{j \neq i}^{3} U_{x, j}^{N} U_{x+j, i}^{N} U_{x+i, j}^{N \dagger}\right],
$$

so that

$$
A=1 / \beta
$$

A convenient measure of the smearing of the source $\psi(x, t)$ in Eq. (B2) is the rms radius

$$
r_{\mathrm{rms}}=\left\langle r^{2}\right\rangle^{(1 / 2)}=\left[\frac{\int d^{3} x|\vec{x}|^{2} \psi^{*}(\vec{x}, t) \psi(\vec{x}, t)}{\int d^{3} x \psi^{*}(\vec{x}, t) \psi(\vec{x}, t)}\right]^{1 / 2},
$$

and Fig. 2 of Ref. [71] shows how $r_{\text {rms }}$ depends on the parameters $N$ and $\alpha$. As one expects from the fact that 
smearing is a random walk governed by the gauge fields, the rms radius is approximately proportional to $\sqrt{N}$. Since the size of the source is nearly independent of $\alpha$ for $\alpha>3$, at which point the constant term in Eq. (B2) becomes negligible relative to the hopping term, in all our calculations, we use $\alpha=3$, which provides the maximum $r_{\text {rms }}$ for a given number of smearing steps $N$.

It is simplest to think about optimization criteria for Wilson fermions, for which one can construct a transfer matrix and correct propagators such that the two-point correlation function has quarks and antiquarks properly normal ordered at zero time separation [72]. In this case, the source may be optimized straightforwardly by maximizing the overlap between the normalized state created by the action of the source $\left|\Psi^{(r)}\right\rangle=\mathcal{C}^{-1 / 2} \bar{N}^{(r)}|\Omega\rangle$, where the source $\bar{N}^{(r)}$ has rms radius $r$, and the normalized ground state of the nucleon $|0\rangle$. Denoting the momentum projected normalized eigenstates of the nucleon by $|n\rangle$ and their energies by $E_{n}$, the momentum projected two-point correlation function may be expanded:

$$
\begin{aligned}
C^{(r)}(t) & =\int d^{3} x\left\langle N^{(r)}(x, t) \bar{N}^{(r)}(0,0)\right\rangle \\
& =\mathcal{C} \sum_{n}\left|\left\langle\Psi^{(r)} \mid n\right\rangle\right|^{2} e^{-E_{n} t},
\end{aligned}
$$

where $\mathcal{C}$ is an unknown normalization constant. Since one can directly measure the correlation function at zero time separation

$$
A^{(r)}=C^{(r)}(0)=\mathcal{C} \sum_{n}\left|\left\langle\Psi^{(r)} \mid n\right\rangle\right|^{2},
$$

and reliably fit the large $t$ behavior of the correlation function to extract the ground state contribution

$$
B^{(r)}=\mathcal{C}\left|\left\langle\Psi^{(r)} \mid 0\right\rangle\right|^{2},
$$

the probability that the source contains the nucleon ground state is given by

$$
\mathcal{P}^{(r)}=\frac{B^{(r)}}{A^{(r)}}=\left|\left\langle\Psi^{(r)} \mid 0\right\rangle\right|^{2} .
$$

Using this criterion, Bratt [73] has recently shown that optimizing the source size with 4-component nucleon sources yields a maximum overlap of $35 \%$, projecting onto the upper two components (in the Bjorken-Drell convention for which these components yield the nonrelativistic limit) increases the overlap to 50\%, and APE smearing of the gauge links in the source further increases the overlap to $80 \%$.

For domain wall fermions, which do not have a local transfer matrix, we consider the following generalization of Eqs. (B11)-(B13), which compares the ratio of the correlation function and extrapolated ground state contribution at time $t$ instead of time 0 :

$$
A^{(r)}(t)=C^{(r)}(t),
$$

$$
\begin{gathered}
B^{(r)}(t)=\mathcal{C}\left|\left\langle\Psi^{(r)} \mid 0\right\rangle\right|^{2} e^{-E_{0} t}, \\
\mathcal{P}^{(r)}(t)=\frac{B^{(r)}(t)}{A^{(r)}(t)} .
\end{gathered}
$$

This ratio, $\mathcal{P}^{(r)}(t)$, ranges from the overlap $\mathcal{P}^{(r)}$ at $t=0$ to 1 in the limit $t \rightarrow \infty$. We expect that for small $t$, it is still a good measure of the presence of excited state components in the source and should have a maximum close to the maximum in $\mathcal{P}^{(r)}$. This expectation is borne out in the case of Wilson fermions, and we note that this criterion gets even better as the lattice spacing decreases. Since we are only interested in the dependence of $\mathcal{P}^{(r)}(t)$ on the rms radius $r$ and the absolute normalization for $t \neq 0$ has no physical significance, it suffices to calculate the following ratio for large $t_{0}$ :

$$
\frac{C^{(r)}\left(t_{0}\right)}{C^{(r)}(t)} \stackrel{t_{0} \rightarrow \infty}{\longrightarrow} \frac{\mathcal{C}\left|\left\langle\Psi^{(r)} \mid 0\right\rangle\right|^{2} e^{-E_{0} t_{0}}}{C^{(r)}(t)}=\mathcal{P}^{(r)}(t) e^{E_{0}\left(t_{0}-t\right)} .
$$

For each value of $t$, it is convenient to normalize the curve such that its maximum value is unity. Hence, defining the rms radius at the maximum as $r^{*}$, our final criterion for optimizing the smearing is the ratio

$$
R^{(r)}(t)=\frac{C^{(r)}\left(t_{0}\right) / C^{(r)}(t)}{C^{\left(r^{*}\right)}\left(t_{0}\right) / C^{\left(r^{*}\right)}(t)} .
$$

Equation (B18) has the computational advantages that all oscillating terms in the time dependence of the correlation functions cancel out of the ratios and that jackknife or bootstrap analysis enables accurate measurements on small ensembles.

We now show the results of optimizing the ratio $R^{(r)}(t)$ on a coarse $24^{3} \times 64$ domain wall lattice with $a=$ $0.114 \mathrm{fm}, m_{\pi}=420 \mathrm{MeV}, m_{s}=0.04$, and $m_{u}=0.01$, using 32 configurations and on a fine $32^{3} \times 64$ domain wall lattice with $a=0.084 \mathrm{fm}, m_{\pi}=297 \mathrm{MeV}, m_{s}=$ 0.03 , and $m_{u}=0.004$ using 33 configurations. We included both APE smearing, with $\beta=0.3509$, and Wuppertal smearing with $\alpha=3$. Because APE smearing smooths the links, the rms radius obtained from a given number of Wuppertal steps changes with the number of APE steps, becoming slightly larger as the number of APE smears increases. Figure 20 shows the rms radius calculated as a function of both the number of APE and Wuppertal steps for both lattice spacings.

Since fluctuations in the normalization of the source directly contribute to the overall fluctuations in correlation functions, it is desirable to use APE smearing to smooth the spatial links used in generating the source and thereby diminish the fluctuations. A simple measure of these fluctuations is the relative fluctuation $\frac{\delta \mathcal{O}}{\mathcal{O}}=\frac{\left\langle(\mathcal{O}-\langle\mathcal{O}\rangle)^{2}\right\rangle^{(1 / 2)}}{\langle\mathcal{O}\rangle}$, where $\mathcal{O}$ is the rms radius defined in Eq. (B9). Figure 21 shows the dramatic effect that APE smearing has in reducing these fluctuations for both lattice spacings. Since the in- 


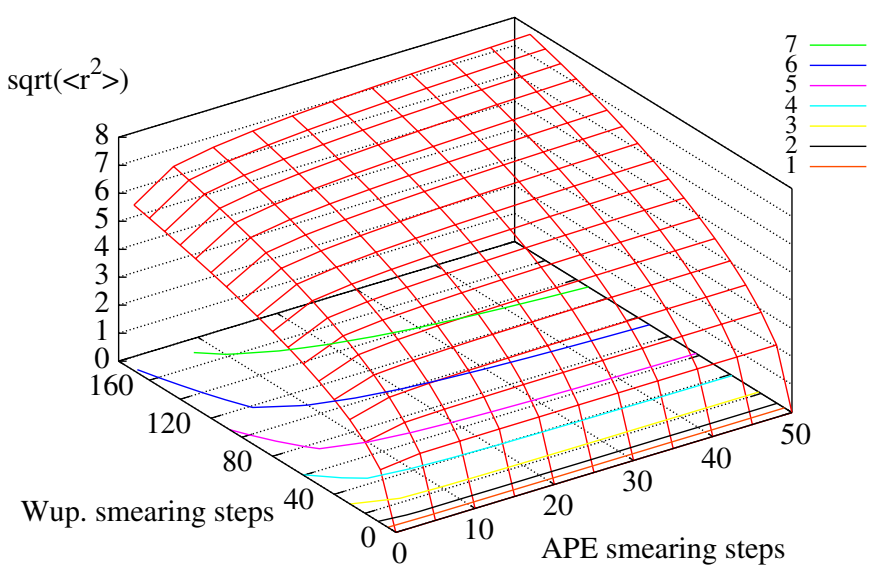

(a)

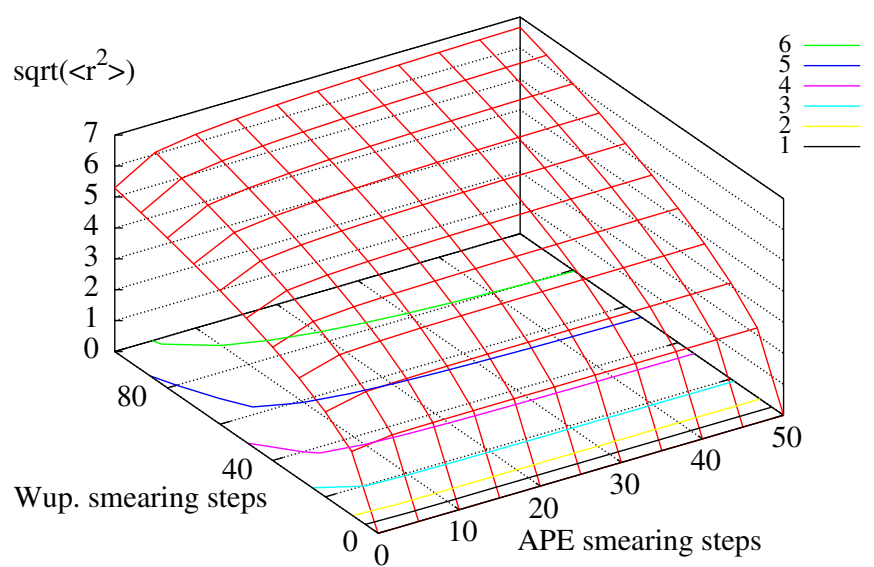

(b)

FIG. 20 (color online). The rms radius of a gauge invariant smeared source as a function of the coefficient $\alpha$ and number of smearing steps $N$ defined in Eq. (B2) for the coarse (a) and fine (b) lattices. The curves projected in the horizontal plane show the numbers of smearing steps required for the specific values of rms radii shown in the key.

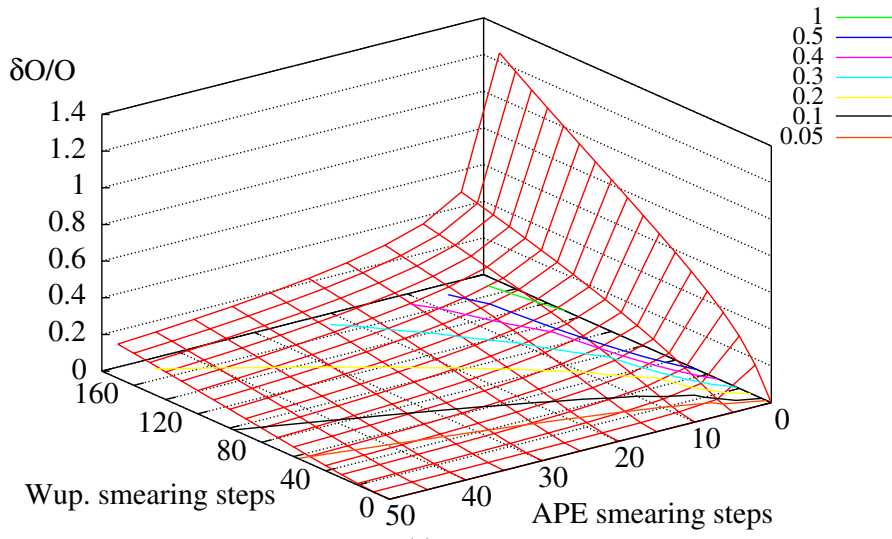

(a)

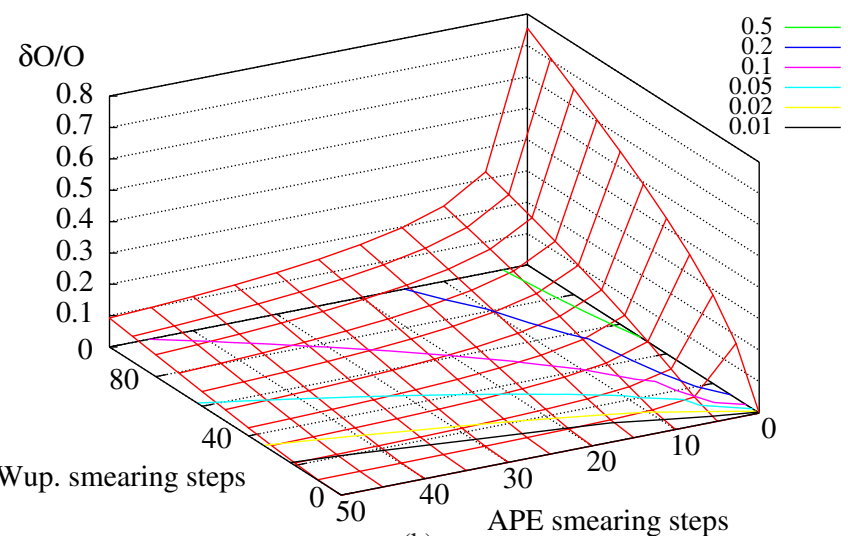

(b)

FIG. 21 (color online). The source variance $\delta \mathcal{O} / \mathcal{O}$ as a function of the rms radius and number of APE smears $N$ for the coarse (a) and fine (b) lattices. The curves projected in the horizontal plane show the numbers of smearing steps required for the specific values of source variance shown in the key.

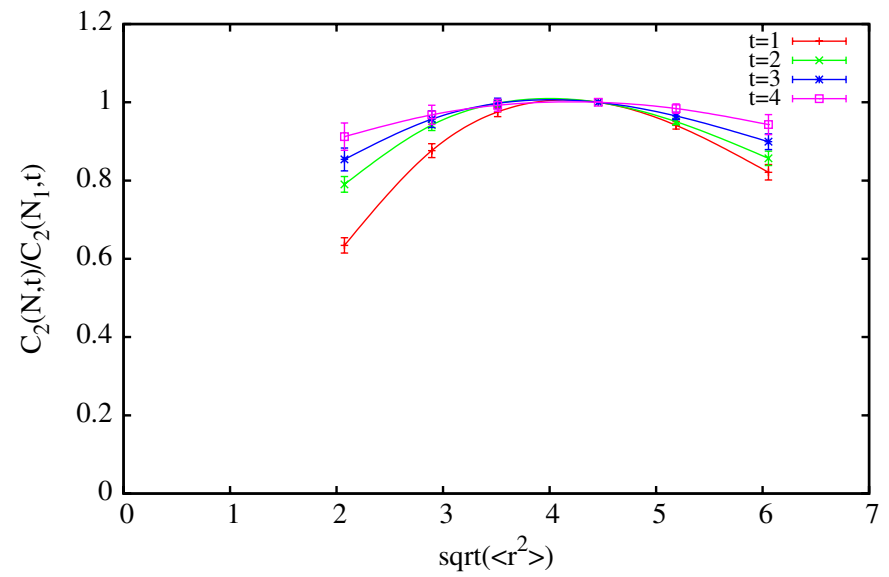

(a)

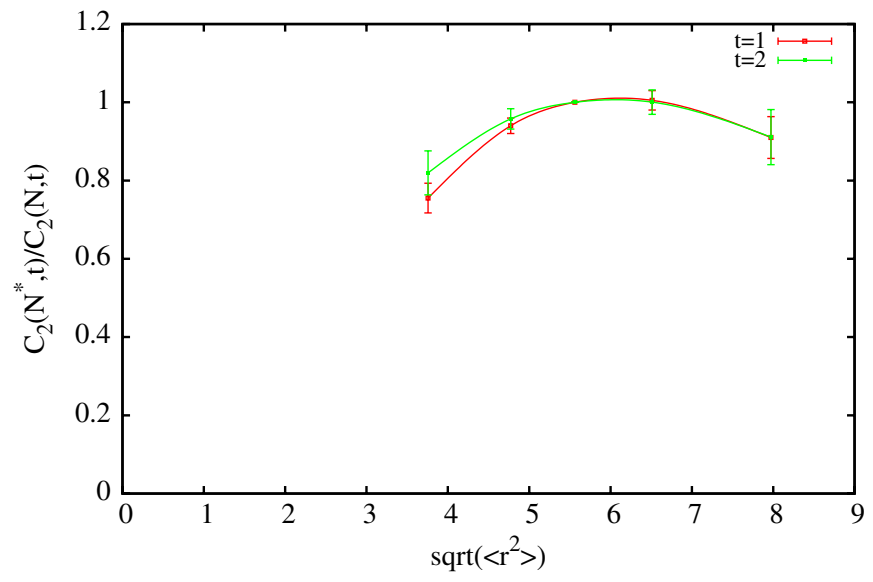

(b)

FIG. 22 (color online). Panel (a) shows the ratio $R^{(r)}(t)$ for $t=1,2,3$, and 4 as a function of $r$ on the coarse lattice. The solid curves are splines passing through the mean values to guide the eye. This graph provides a robust determination of the optimal rms radius $r=4.0$ lattice units, corresponding to $N=40$ Wuppertal smearing steps. Panel (b) shows the analogous ratio $R^{(r)}(t)$ for $t=1$ and 2 as a function of $r$ on the fine lattice. 
cremental benefit of successive smearing becomes small beyond 25 smearing steps, we have chosen to use 25 steps throughout. Note that for the largest number of Wuppertal steps, this reduces the noise by a factor of more than 5 in each case.

Figure 22 shows the primary result of the calculation for both lattice spacings. For the coarse lattice, the ratio $R^{(r)}(t)$ is calculated at six values of the number of Wuppertal steps, $N=10,20,30,50,70,100$, corresponding to rms radii, $r=2.07,2.89,3.51,4.46,5.19$, and 6.06 lattice units, respectively. We chose $r^{*}=4.46 \mathrm{fm}$ and calculated bootstrap error bars using 32 configurations. Instead of normalizing at a single value of $t_{0}$ as in Eq. (B18), the errors in the ratios in Fig. 22 were further reduced by normalizing to an exponential fit to each correlation function in the region $t=[6-12]$. These results are completely consistent with those of a single $t_{0}$, but display the shape of the maxima more precisely. Note that for all four values $t=1,2,3$, and 4 , the curves are accurately determined and the ratio $R^{(r)}(t)$ has a maximum at approximately the same point, $r=4.0$, corresponding to $N=40$. Thus, we believe our optimization criterion is robust and statistically accurate for domain wall fermions.

For the fine lattices, the ratio $R^{(r)}(t)$ is calculated at 5 values of the number of Wuppertal steps, $N=30,50,70$,
TABLE XVIII. Parameters for optimal sources.

\begin{tabular}{lccccccc}
\hline \hline Lattice & \multicolumn{3}{c}{ APE smearing } & \multicolumn{3}{c}{ Wuppertal smearing } & Size \\
$a(\mathrm{fm})$ & $\beta$ & $\mathrm{A}$ & $N_{\mathrm{APE}}$ & $\alpha$ & $\sigma$ & $N_{W}$ & $\left\langle r^{2}\right\rangle^{1 / 2}$ \\
\hline 0.114 & 0.3509 & 2.85 & 25 & 3 & 5.026 & 40 & 4.0 \\
0.084 & 0.3509 & 2.85 & 25 & 3 & 7.284 & 84 & 6.0 \\
\hline \hline
\end{tabular}

100 , and 150 , corresponding to $\mathrm{rms}$ radii $r=3.76,4.77$, 5.56, 6.51, and 7.77 lattice units, respectively. We chose $r^{*}=5.56$, normalized by exponential fits to each correlation function in the region $t=[6: 12]$, calculated jackknife error bars, and only included $t=1$ and 2 to avoid making the graph confusing due to the larger error bars. The maximum occurs at approximately $r=6.0$ lattice units, corresponding to 84 Wuppertal smearing steps. This result appears reasonable, since assuming a constant rms radius in physical units would imply that the rms radius on the coarser lattice of 4.0 lattice units would scale to $4.0 \times$ $0.114 / 0.084=5.4$ lattice units on the present lattice, and the pion mass on the finer lattice is somewhat lighter.

We summarize the final parameters for optimal sources used in this work in Table XVIII, where the parameters are defined in Eqs. (B3)-(B8).
[1] M. Burkardt, Phys. Rev. D 62, 071503(R) (2000); 66, 119903(E) (2002).

[2] M. Burkardt, Int. J. Mod. Phys. A 18, 173 (2003).

[3] J. Friedrich and T. Walcher, Eur. Phys. J. A 17, 607 (2003).

[4] J. Arrington, W. Melnitchouk, and J. A. Tjon, Phys. Rev. C 76, 035205 (2007).

[5] G. Höhler et al., Nucl. Phys. B114, 505 (1976).

[6] P. Mergell, U. G. Meißner, and D. Drechsel, Nucl. Phys. A596, 367 (1996).

[7] M.A. Belushkin, H.W. Hammer, and U.-G. Meißner, Phys. Rev. C 75, 035202 (2007).

[8] J. C. Bernauer, Lect. Notes Phys. 745, 79 (2008).

[9] B. D. Milbrath et al. (Bates FPP Collaboration), Phys. Rev. Lett. 80, 452 (1998).

[10] T. Pospischil et al. (A1 Collaboration), Eur. Phys. J. A 12, 125 (2001).

[11] O. Gayou et al. (Jefferson Lab Hall A Collaboration), Phys. Rev. Lett. 88, 092301 (2002).

[12] O. Gayou et al., Phys. Rev. C 64, 038202 (2001).

[13] V. Punjabi et al., Phys. Rev. C 71, 055202 (2005).

[14] J. Arrington et al., arXiv:nucl-ex/0408020.

[15] OLYMPUS Collaboration, http://web.mit.edu/olympus/ (2009).

[16] M. Göckeler et al. (QCDSF Collaboration), Phys. Rev. D 71, 034508 (2005).

[17] C. Alexandrou, G. Koutsou, J. W. Negele, and A. Tsapalis,
Phys. Rev. D 74, 034508 (2006).

[18] S. Sasaki and T. Yamazaki, Phys. Rev. D 78, 014510 (2008).

[19] A. Tang, W. Wilcox, and R. Lewis, Phys. Rev. D 68, 094503 (2003).

[20] S. Boinepalli, D. B. Leinweber, A. G. Williams, J. M. Zanotti, and J.B. Zhang, Phys. Rev. D 74, 093005 (2006).

[21] M. Göckeler et al. (QCDSF/UKQCD Collaboration), Proc. Sci., LAT2007 (2007) 161 [arXiv:0710.2159].

[22] C. Alexandrou, arXiv:0906.4137.

[23] C. Alexandrou et al. (COL-NOTE = European Twisted Mass Collaboration), arXiv:0811.0724.

[24] H.-W. Lin, T. Blum, S. Ohta, S. Sasaki, and T. Yamazaki, Phys. Rev. D 78, 014505 (2008).

[25] P. Hägler et al. (LHPC Collaboration), Phys. Rev. D 77, 094502 (2008).

[26] J.D. Bratt et al. (LHPC Collaboration), Proc. Sci., LATTICE2008 (2008) 141 [arXiv:0810.1933].

[27] J. D. Bratt et al. (LHPC Collaboration) (unpublished).

[28] S. Ohta and T. Yamazaki (for RBC and UKQCD Collaborations), arXiv:0810.0045.

[29] T. Yamazaki et al., Phys. Rev. D 79, 114505 (2009).

[30] S. N. Syritsyn et al., Proc. Sci., LATTICE2008 (2008) 169 [arXiv:0903.3063].

[31] T. R. Hemmert, B. R. Holstein, and J. Kambor, J. Phys. G 24, 1831 (1998). 
[32] M. Dorati, T. A. Gail, and T. R. Hemmert, Nucl. Phys. A798, 96 (2008).

[33] T. Gail, Ph.D. thesis, Technical University Munich, 2007.

[34] T. Becher and H. Leutwyler, Eur. Phys. J. C 9, 643 (1999).

[35] P. Wang, D. B. Leinweber, A. W. Thomas, and R.D. Young, Phys. Rev. D 75, 073012 (2007).

[36] P. Wang, D. B. Leinweber, A. W. Thomas, and R.D. Young, Phys. Rev. D 79, 094001 (2009).

[37] C. Allton et al. (RBC-UKQCD Collaboration), Phys. Rev. D 78, 114509 (2008).

[38] S. Güsken et al., Phys. Lett. B 227, 266 (1989).

[39] S. Güsken, Nucl. Phys. B, Proc. Suppl. 17, 361 (1990).

[40] M. Falcioni, M. L. Paciello, G. Parisi, and B. Taglienti, Nucl. Phys. B251, 624 (1985).

[41] S. R. Sharpe, arXiv:0706.0218.

[42] T. Blum et al., Phys. Rev. D 69, 074502 (2004).

[43] J. Gasser and H. Leutwyler, Ann. Phys. (N.Y.) 158, 142 (1984).

[44] V. Bernard, H. W. Fearing, T. R. Hemmert, and U. G. Meißner, Nucl. Phys. A635, 121 (1998).

[45] L. Del Debbio, L. Giusti, M. Lüscher, R. Petronzio, and N. Tantalo, J. High Energy Phys. 02 (2007) 056.

[46] P. Dimopoulos et al. (ETM Collaboration), arXiv: 0810.2873 .

[47] G. Colangelo, J. Gasser, and H. Leutwyler, Nucl. Phys. B603, 125 (2001).

[48] J. Bijnens, G. Colangelo, and P. Talavera, J. High Energy Phys. 05 (1998) 014.

[49] B. Kubis and U.-G. Meißner, Nucl. Phys. A679, 698 (2001).

[50] T. Bakeyev et al. (QCDSF-UKQCD Collaboration), Phys. Lett. B 580, 197 (2004).

[51] J. J. Kelly, Phys. Rev. C 70, 068202 (2004).

[52] T. R. Hemmert and W. Weise, Eur. Phys. J. A 15, 487 (2002).

[53] G. Colangelo and S. Dürr, Eur. Phys. J. C 33, 543 (2004).
[54] A. Walker-Loud et al., Phys. Rev. D 79, 054502 (2009).

[55] V. Bernard, T. R. Hemmert, and U.-G. Meißner, Phys. Lett. B 622, 141 (2005).

[56] C. Amsler et al. (Particle Data Group), Phys. Lett. B 667, 1 (2008).

[57] T. R. Hemmert, M. Procura, and W. Weise, Phys. Rev. D 68, 075009 (2003).

[58] R. G. Edwards et al. (LHPC Collaboration), Phys. Rev. Lett. 96, 052001 (2006).

[59] M. Procura, B. U. Musch, T. R. Hemmert, and W. Weise, Phys. Rev. D 75, 014503 (2007).

[60] A. Ali Khan et al., Phys. Rev. D 74, 094508 (2006).

[61] R. M. Davidson, N. C. Mukhopadhyay, and R. S. Wittman, Phys. Rev. D 43, 71 (1991).

[62] T. R. Hemmert, B. R. Holstein, and J. Kambor, Phys. Lett. B 395, 89 (1997).

[63] J. Gasser, M.E. Sainio, and A. Svarc, Nucl. Phys. B307, 779 (1988).

[64] T. A. Gail and T. R. Hemmert (unpublished).

[65] V. Bernard, N. Kaiser, and U.-G. Meißner, Nucl. Phys. A615, 483 (1997).

[66] N. Fettes, U.-G. Meißner, and S. Steininger, Nucl. Phys. A640, 199 (1998).

[67] D. R. Entem and R. Machleidt, Phys. Rev. C 66, 014002 (2002).

[68] M. Procura, B. U. Musch, T. Wollenweber, T. R. Hemmert, and W. Weise, Phys. Rev. D 73, 114510 (2006).

[69] A. Ali Khan et al. (QCDSF-UKQCD Collaboration), Nucl. Phys. B689, 175 (2004).

[70] S. Syritsyn and J. W. Negele, Proc. Sci., LAT2007 (2007) 078 [arXiv:0710.0425].

[71] D. Dolgov et al. (LHPC Collaboration), Phys. Rev. D 66, 034506 (2002).

[72] M. Lüscher, Commun. Math. Phys. 54, 283 (1977).

[73] J. Bratt and J. W. Negele, arXiv:0810.1954.

[74] R. G. Edwards and B. Joo (SciDAC Collaboration), Nucl. Phys. B, Proc. Suppl. 140, 832 (2005). 